
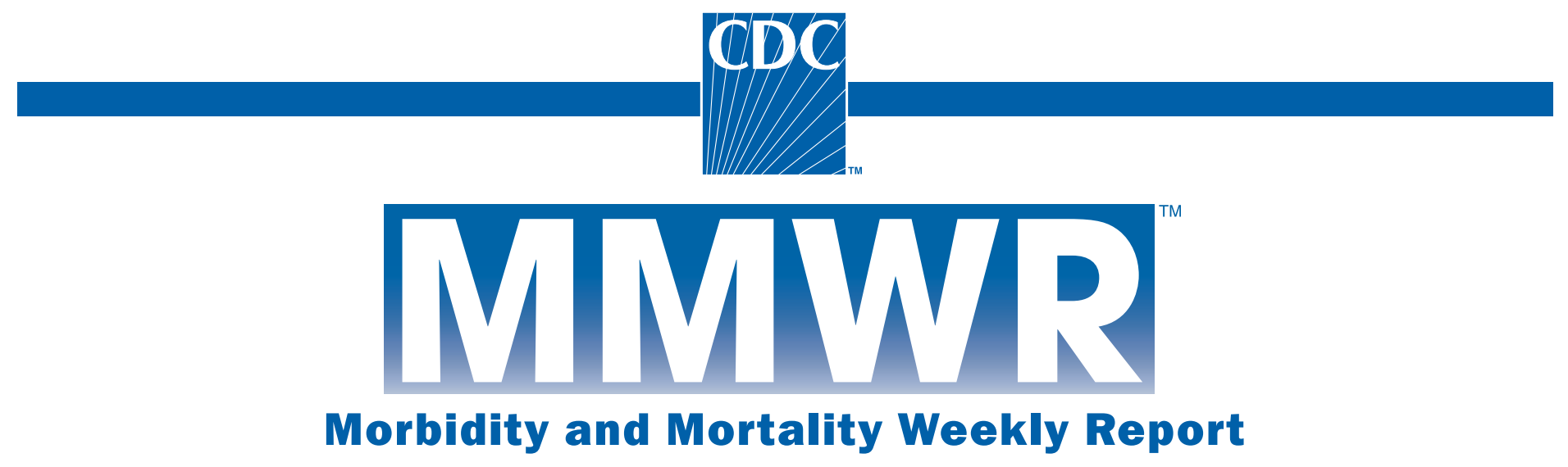

\title{
Weekly
}

\section{Fatalities and Injuries from Falls Among Older Adults - United States, 1993-2003 and 2001-2005}

Unintentional falls are a common occurrence among older adults, affecting approximately $30 \%$ of persons aged $\geq 65$ years each year (1). The injuries received from a fall can result in death, disability, nursing-home admission, and direct medical costs (2,3). In 2003, a total of 13,700 persons aged $\geq 65$ years died from falls, and 1.8 million were treated in emergency departments (EDs) for nonfatal injuries from falls.* Falls cause the majority of hip fractures, which often result in longterm functional impairments that might require admission to a nursing home for a year or more (2). To examine trends in fatal and nonfatal falls among older persons, CDC analyzed U.S. rates of 1) fatalities from falls (during 1993-2003), 2) hospitalizations for hip fractures (1993-2003), and 3) nonfatal injuries resulting from falls in persons treated in EDs (2001-2005). This report summarizes the results of those analyses, which indicated that, during 1993-2003, the overall rate of fatal falls among persons aged $\geq 65$ years increased, and the rate of hospitalizations for hip fractures decreased; during 2001-2005, the change in the overall rate of nonfatal injuries from falls was not statistically significant. However, disparities by sex existed for all three measures. Certain interventions can reduce falls (e.g., exercising regularly or having medicines reviewed to reduce side effects and interactions), but implementation at the community level remains limited (2), and additional measures are needed to promote widespread adoption.

Data on fatal falls that occurred during 1993-2003 were obtained from annual mortality data of the Vital Statistics of the United States (4). Cause-of-death data were based on information from death certificates completed by attending physicians, medical examiners, or coroners. Fall-related

\footnotetext{
*Web-based injury statistics query and reporting system. Available at http://www.
} cdc.gov/ncipc/wisqars. deaths for 1993-1998 were defined as those deaths with an underlying cause coded E880-E886.9 or E888, according to the International Classification of Diseases, Ninth Revision (ICD-9); for 1999-2003, fall-related deaths were defined as those deaths coded W00-W19 according to the Tenth Revision (ICD-10) (5).

National estimates of hospital admissions for hip fractures that occurred during 1993-2003 were obtained from the $\mathrm{Na}$ tional Hospital Discharge Survey (NHDS), which collects data from a sample of inpatient records acquired from a national probability sample of nonfederal, short-stay hospitals; data represent a sample of hospital discharges. Hospitalizations for hip fractures include cases with any diagnosis coded 820, according to the International Classification of Diseases, Ninth Revision, Clinical Modification (ICD-9-CM) (G).

Data on nonfatal injuries from falls that occurred during 2001-2005 were obtained from the National Electronic Injury Surveillance System-All Injury Program (NEISS-AIP), which is operated by the Consumer Product Safety Commission and collects data regarding initial visits for all types and causes of injuries in persons treated in EDs. These data are drawn from a nationally representative sample of 66 hospitals, selected as a stratified probability sample of hospitals in the United States (7). Information about the most severe injury for each case is collected from the medical record; data
INSIDE
1224 Self-Rated Fair or Poor Health Among Adults with Diabetes — United States, 1996-2005
1227 Hazardous Materials Release Resulting from Home Production of Biodiesel - Colorado, May 2006
1228 Notice to Readers
1229 QuickStats 
The MMWR series of publications is published by the Coordinating Center for Health Information and Service, Centers for Disease Control and Prevention (CDC), U.S. Department of Health and Human Services, Atlanta, GA 30333.

Suggested Citation: Centers for Disease Control and Prevention. [Article title]. MMWR 2006;55:[inclusive page numbers].

\section{Centers for Disease Control and Prevention}

Julie L. Gerberding, MD, MPH Director

Tanja Popovic, MD, PhD

(Acting) Chief Science Officer

James W. Stephens, PhD

(Acting) Associate Director for Science

Steven L. Solomon, MD

Director, Coordinating Center for Health Information and Service

Jay M. Bernhardt, PhD, MPH

Director, National Center for Health Marketing Judith R. Aguilar

(Acting) Director, Division of Health Information Dissemination (Proposed)

\section{Editorial and Production Staff}

John S. Moran, MD, MPH

(Acting) Editor, MMWR Series

Suzanne M. Hewitt, MPA

Managing Editor, MMWR Series

Douglas W. Weatherwax

(Acting) Lead Technical Writer-Editor

Catherine H. Bricker, MS

Jude C. Rutledge

Writers-Editors

Beverly J. Holland

Lead Visual Information Specialist

Lynda G. Cupell

Malbea A. LaPete

Visual Information Specialists

Quang M. Doan, MBA

Erica R. Shaver

Information Technology Specialists

\section{Editorial Board}

William L. Roper, MD, MPH, Chapel Hill, NC, Chairman

Virginia A. Caine, MD, Indianapolis, IN

David W. Fleming, MD, Seattle, WA

William E. Halperin, MD, DrPH, MPH, Newark, NJ

Margaret A. Hamburg, MD, Washington, DC

King K. Holmes, MD, PhD, Seattle, WA

Deborah Holtzman, PhD, Atlanta, GA

John K. Iglehart, Bethesda, MD

Dennis G. Maki, MD, Madison, WI

Sue Mallonee, MPH, Oklahoma City, OK

Stanley A. Plotkin, MD, Doylestown, PA

Patricia Quinlisk, MD, MPH, Des Moines, IA

Patrick L. Remington, MD, MPH, Madison, WI

Barbara K. Rimer, DrPH, Chapel Hill, NC

John V. Rullan, MD, MPH, San Juan, PR Anne Schuchat, MD, Atlanta, GA

Dixie E. Snider, MD, MPH, Atlanta, GA John W. Ward, MD, Atlanta, GA are weighted by the inverse probability of selection and summed to produce national estimates.

Denominators for rates of fatal falls, hip fractures, and nonfatal injuries from falls were calculated using U.S. Census population estimates, ${ }^{\dagger}$ and rates were age adjusted to the 2000 U.S. standard population. Weighted least squares regression was used to test for linear trend (as the percentage change in annual rates); differences with $\mathrm{p}<0.05$ were considered statistically significant $(8)$.

During 1993-2003, the age-adjusted rate of fatalities from falls increased significantly, and rates were significantly higher among men compared with women (Table 1). Fatality rates increased both for men (from 31.8 per 100,000 population to 46.2, an increase of 45.3\%) ( $\mathrm{p}<0.01)$ and women (from 19.5 per 100,000 population to 31.1 , an increase of $59.5 \%)(\mathrm{p}<0.01)$. During 1993-2003, rates increased in all racial populations for both sexes, with the exception of black men, whose rate was unchanged. In 2003, rates varied by race among both men (whites: 48.3 per 100,000 population; Asians/Pacific Islanders [A/PI]: 36.6; and blacks: 22.3) and women (whites: 32.8 per 100,000 population; A/PI: 23.2; and blacks: 13.9 ).

During 1993-2003, the overall age-adjusted hospitalization rate for hip fractures decreased by $15.5 \%$, from 917.6 per 100,000 population to 775.7 ( $\mathrm{p}=0.001$ test for trend) (Table 1). The hospitalization rate increased to 990.5 per 100,000 population during 1993-1996, before declining. During 1993-2003, rates differed by sex. The annual rate for women was $52 \%-119 \%$ higher than the rate for men. However, the hospitalization rate for hip fractures did not increase significantly $(5.7 \%, \mathrm{p}=0.53)$ for men during 1993-2003 and declined $20.8 \%(\mathrm{p}<0.01)$ for women.

During 2001-2005, neither the change in the overall rate of nonfatal injury from falls nor any of the changes by sex or race were significant (Table 2). In contrast to fatal falls, annual rates of nonfatal injuries for women were, on average, $48.4 \%$ higher than the rates for men. Comparing rates for fatal falls and nonfatal injuries from falls during the most recent 3-year period (2001-2003) when data for both were available, the rate for fatal falls increased $13.3 \%$ ( $9.8 \%$ for men and $15.6 \%$ for women), whereas the rates for nonfatal injuries increased 7.6\% (7.5\% for men and 7.9\% for women).

Reported by: JA Stevens, PhD, Div of Unintentional Injury Prevention; G Ryan, PhD, Office of Statistics and Programming; M Kresnow, MS, Office of Statistics and Programming, National Center for Injury Prevention and Control, CDC.

Editorial Note: This study examined trends in rates of fatal falls and hospitalizations for hip fractures during 1993-2003

$\dagger$ U.S. Census Bureau population projections. Available at http://www.census.gov/ population/www/projections/popproj.html. 
TABLE 1. Age-adjusted* rates ${ }^{\dagger}$ of fatal falls or hospitalizations for hip fractures among persons aged $\geq 65$ years, by sex and race United States, 1993-2003

\begin{tabular}{|c|c|c|c|c|c|c|c|c|c|c|c|c|}
\hline \multirow[b]{2}{*}{ Event/Characteristic } & \multicolumn{11}{|c|}{ Year } & \multirow{2}{*}{$\begin{array}{r}\% \text { change } \\
1993-2003 \\
\end{array}$} \\
\hline & 1993 & 1994 & 1995 & 1996 & 1997 & 1998 & 1999 & 2000 & 2001 & 2002 & 2003 & \\
\hline \multicolumn{13}{|l|}{ Fatal falls } \\
\hline Both sexes & 23.7 & 23.9 & 24.4 & 26.2 & 27.4 & 28.5 & 29.4 & 29.5 & 32.5 & 35.1 & 36.8 & 55.3 \\
\hline \multicolumn{13}{|l|}{ Sex/Race§ } \\
\hline Men overall & 31.8 & 32.9 & 32.6 & 34.6 & 36.7 & 37.1 & 38.7 & 38.5 & 42.1 & 44.4 & 46.2 & 45.3 \\
\hline White & 32.7 & 33.9 & 33.5 & 36.0 & 38.1 & 38.5 & 39.9 & 40.0 & 43.7 & 46.2 & 48.3 & 47.7 \\
\hline Black & 22.1 & 19.5 & 21.7 & 19.5 & 21.3 & 21.3 & 23.7 & 21.1 & 25.0 & 24.6 & 22.3 & 0.0 \\
\hline Asian/Pacific Islander & 20.9 & 36.3 & 31.9 & 27.2 & 28.8 & 30.6 & 36.9 & 31.0 & 35.1 & 35.9 & 36.6 & 75.1 \\
\hline Women overall & 19.5 & 19.1 & 20.0 & 21.6 & 22.1 & 23.7 & 24.2 & 24.5 & 26.9 & 29.5 & 31.1 & 59.5 \\
\hline White & 20.3 & 20.1 & 20.9 & 22.6 & 23.0 & 24.9 & 25.4 & 25.8 & 28.3 & 31.3 & 32.8 & 61.6 \\
\hline Black & 10.2 & 9.1 & 10.7 & 10.2 & 11.5 & 11.5 & 12.4 & 11.0 & 12.1 & 11.7 & 13.9 & 36.3 \\
\hline Asian/Pacific Islander & 15.0 & 14.4 & 15.5 & 17.4 & 19.1 & 17.8 & 13.6 & 20.4 & 18.0 & 20.9 & 23.2 & 54.7 \\
\hline \multicolumn{13}{|l|}{ Hip fractures } \\
\hline Both sexes & 917.6 & 900.3 & 875.6 & 990.5 & 929.1 & 930.8 & 919.3 & 877.3 & 866.3 & 804.8 & 775.7 & -15.5 \\
\hline Men & 552.3 & 578.0 & 579.6 & 567.1 & 635.7 & 678.9 & 597.3 & 570.6 & 556.3 & 525.1 & 583.6 & 5.7 \\
\hline Women & $1,118.9$ & $1,078.4$ & $1,033.1$ & $1,239.2$ & $1,096.4$ & $1,071.0$ & $1,098.4$ & $1,042.2$ & 1,038.6 & 971.4 & 886.2 & -20.8 \\
\hline
\end{tabular}

SOURCES: Vital Statistics of the United States (fatal falls) and National Hospital Discharge Survey (hip fractures).

${ }^{*}$ Age adjusted to the U.S. standard 2000 population.

Per 100,000 population.

$\S$ Whites were all non-Hispanic; blacks might include Hispanics.

TABLE 2. Age-adjusted ${ }^{*}$ rate $^{\dagger}$ of nonfatal falls among persons aged $\geq 65$ years, by sex and race - United States, 2001-2005

\begin{tabular}{|c|c|c|c|c|c|c|}
\hline \multirow[b]{2}{*}{ Characteristic } & \multicolumn{5}{|c|}{ Year } & \multirow{2}{*}{$\begin{array}{l}\% \text { change } \\
2001-2005\end{array}$} \\
\hline & 2001 & 2002 & 2003 & 2004 & 2005 & \\
\hline Both sexes§ & $4,617.0$ & $4,539.2$ & $4,967.6$ & $4,972.6$ & $4,746.8$ & 2.8 \\
\hline \multicolumn{7}{|l|}{ Sex/Raceףl } \\
\hline Men overall & $3,590.0$ & $3,490.6$ & $3,859.4$ & $3,847.6$ & $3,674.0$ & 2.3 \\
\hline White & $3,090.3$ & $2,920.5$ & $3,278.6$ & $3,133.8$ & $2,823.6$ & -8.6 \\
\hline Black & $2,813.8$ & $3,270.4$ & $3,114.4$ & $3,521.6$ & $3,033.6$ & 7.8 \\
\hline Women overall & $5,283.0$ & $5,238.0$ & $5,697.8$ & $5,712.2$ & $5,466.7$ & 3.5 \\
\hline White & $4,478.2$ & $4,348.3$ & $4,760.4$ & $4,611.3$ & $4,223.2$ & -5.7 \\
\hline Black & $4,914.3$ & $4,828.8$ & $4,752.5$ & $5,229.3$ & $4,595.7$ & -6.5 \\
\hline
\end{tabular}

SOURCE: National Electronic Injury Surveillance System-All Injury Program.

${ }^{*}$ Age adjusted to the U.S. standard 2000 population.

$\dagger$ Per 100,000 population.

$\S$ Includes persons with missing data regarding race.

"Whites were all non-Hispanic; blacks might include Hispanics.

and in rates of nonfatal injuries resulting from falls during 2001-2005. The findings indicate that rates of fatal falls increased significantly among both men and women but were consistently higher among men. Whites had the highest fatal fall rates, but an increasing trend was observed for all races. Changes in rates for nonfatal injuries from falls were not statistically significant.

Although only 3 years of rates for fatal falls and nonfatal injuries could be compared directly, the greater increase in the fatal falls rate can be partly explained by the increase in injurycausing falls overall. In addition, although fatal fall rates are age adjusted, residents of the United States are living longer in large part because of decreasing mortality from chronic conditions (e.g., heart disease, cancer, or stroke). The U.S. life expectancy increased from 75.5 years in 1993 to 77.6 years in 2003 (9). These changes have resulted in a U.S. population with a greater proportion of older adults who are living with chronic diseases, leaving them at greater risk for falling and less likely to survive the injuries resulting from a fall.

Rates of nonfatal injuries from falls and particularly rates of hospitalizations for hip fractures were higher among women than men. However, hospitalization rates for hip fractures appear to be declining among women. Older women are disproportionately affected by osteoporosis, a disease in which bones become porous and susceptible to fracture (2). In recent years, osteoporosis screening for women and effective treatments to rebuild bone mass have become widespread (10). These public health measures might be reflected in the lower rates for fractures. Men tend to have greater bone mass and consequently less risk for hip fractures. However, men do sustain hip fractures, especially after age 80 years; the hipfracture rate among men has not decreased and might be 
increasing. Screening and osteoporosis treatment might be broadened to include older men.

The findings in this report are subject to at least five limitations. First, three different data sources were used for the three rates analyzed (i.e., fatalities from falls, hospitalizations for hip fractures, and nonfatal injuries from falls in patients treated in EDs); therefore, these data might not be comparable. Second, racial categories used to analyze fatalities and nonfatal injuries differed. Third, only 5 years of NEISS-AIP data were available; therefore, the same period analyzed for fatality and hip fracture rates could not be used for nonfatal injuries from falls. Fourth, the rate of nonfatal injuries from falls likely was underestimated because only persons treated in hospital EDs were included and not those treated in outpatient settings such as clinics or physician offices. Finally, NHDS reports the number of hospital admissions, not patients; therefore, certain persons seeking treatment for hip fractures might have been counted more than once.

Research has identified interventions that can reduce falls, but development and implementation of community-based programs remains limited (2). Additional measures are needed to successfully disseminate effective fall-prevention programs and to promote widespread adoption at the local level. To help prevent falls among older adults, CDC, in partnership with the CDC Foundation and MetLife Foundation, has produced four posters and updated and redesigned two brochures. What YOU Can Do to Prevent Falls outlines four key fallprevention strategies: exercising regularly, having medications reviewed to reduce side effects and interactions, having yearly eye examinations, and reducing fall hazards in the home. Check for Safety: A Home Fall Prevention Checklist for Older Adults guides readers through a room-by-room check of their homes to find and fix hazards that can increase the risk for falling. The brochures and posters are offered in English, Spanish, and Chinese and are available at http://www.cdc.gov/ncipc/ pub-res/toolkit/brochures.htm. Additional information about CDC's fall-prevention activities is available at http://www.cdc. gov/ncipc/pub-res/toolkit/toolkit.htm.

\section{References}

1. Hausdorff JM, Rios DA, Edelberg HK. Gait variability and fall risk in community-living older adults: a 1-year prospective study. Arch Phys Med Rehabil 2001;82:1050-6.

2. Stevens JA. Falls among older adults—risk factors and prevention strategies. In: Falls free: promoting a national falls prevention action plan. Washington, DC: The National Council on the Aging; 2005.

3. Stevens JA, Corso PS, Finkelstein EA, Miller TR. The costs of fatal and nonfatal falls among older adults. Inj Prev 2006;12:290-5.

4. CDC. Vital statistics of the United States. Hyattsville, MD: US Department of Health and Human Services, CDC; 1993-2003. Available at http://www.cdc.gov/nchs/products/pubs/pubd/vsus/vsus.htm.
5. World Health Organization. International statistical classification of diseases and related health problems. 10th revision. Geneva, Switzerland: World Health Organization; 1992.

6. International classification of diseases. 9th revision, clinical modification. Ann Arbor, MI: Commission on Professional and Hospital Activities; 1980.

7. Schroeder T, Ault K. National Electronic Injury Surveillance SystemAll Injury Program: sample design and implementation. Bethesda, MD: US Consumer Product Safety Commission; 2001.

8. CDC. Trends in hospital utilization: United States, 1988-92. Vital Health Stat 1996;13(124):62-4.

9. CDC. National Nursing Home Survey (NNHS) public-use data files. Hyattsville, MD: US Department of Health and Human Services, CDC. Available at http://www.cdc.gov/nchs/products/elec_prods/subject/nnhs.htm.

10. Ensrud KE, Black DM, Palermo L, et al. Treatment with alendronate prevents fractures in women at highest risk: results from the Fracture Intervention Trial. Arch Intern Med 1997;157:2617-24.

\section{Self-Rated Fair or Poor Health Among Adults with Diabetes - United States, 1996-2005}

Diabetes mellitus affects nearly 21 million persons in the United States (1). Maintaining and improving health-related quality of life among persons with diabetes is a public health goal. Healthy People 2010 includes self-rated health as one of three surveillance tools that can be used to measure healthrelated quality of life (2). To assess the prevalence of self-rated fair or poor health among U.S. adults with diabetes and to identify factors associated with fair or poor health, CDC analyzed 1996-2005 Behavioral Risk Factor Surveillance System (BRFSS) data. This report summarizes the findings of that analysis, which indicated that self-rated fair or poor health was three times more common among adults with diabetes than among those without diabetes and that the prevalence increased during 1996-2005 among young adults (i.e., aged 18-44 years) with diabetes. The results underscore the need for 1) continued interventions to promote healthy behaviors and prevent diabetes and 2) interventions for persons with diabetes to help them better manage their diabetes and prevent diabetes complications, which can increase their perceived quality of life.

BRFSS is an ongoing, state-based, random-digit-dialed telephone survey of the U.S. civilian, noninstitutionalized population aged $\geq 18$ years; the survey is conducted in all 50 states, the District of Columbia, and three U.S. territories. The median state response rate (i.e., the percentage of persons who completed interviews among all eligible persons, including those who were not successfully contacted) was $63.1 \%$ (range: 45.6\%-87.1\%) in 1996 and 51.1\% (range: 34.6\%-67.4\%) 
in 2005. The median cooperation rate (i.e., percentage of persons who completed interviews among all eligible persons who were contacted) was 68.2\% (range: 46.1\%-91.4\%) in 1996 and $75.1 \%$ (range: $58.7 \%-85.3 \%$ ) in 2005 . Persons with diabetes were defined as respondents who answered yes to the question, "Have you ever been told by a doctor that you have diabetes?" Women who were told that they had diabetes only during pregnancy and respondents with prediabetes or borderline diabetes were classified as not having diabetes. Persons with fair or poor health status were defined as those who responded "fair" or "poor" to the question, "Would you say that in general your health is excellent, very good, good, fair, or poor?" Respondents who reported "don't know/not sure" or "refused" were excluded from the analysis. Age-adjusted prevalence was estimated according to the 2000 U.S. standard population. Linear regression analysis was used to assess the trend of self-rated fair or poor health during 1996-2005. Logistic regression analysis was conducted to examine the association between self-rated fair or poor health and selected characteristics. Estimates were weighted to reflect the age, sex, and racial/ethnic distribution of the U.S. population. The statistical significance level was $\mathrm{p}<0.05$.

During 2005, an estimated $49.3 \%$ (95\% confidence interval $[\mathrm{CI}]=48.2 \%-50.5 \%)$ of adults with diabetes aged $\geq 18$ years reported having fair or poor health. After adjusting for respondent age, the prevalence of fair or poor health among adults with diabetes was $46.7 \%(\mathrm{CI}=44.7 \%-48.7 \%)$, more than three times the rate among adults without diabetes $(14.2 \%, \mathrm{CI}=13.9 \%-14.4 \%)$. During 1996-2005, no significant change was identified in the overall prevalence of selfrated fair or poor health among adults with diabetes, although the prevalence did vary by age group. The prevalence did not change for persons aged $\geq 45$ years but increased significantly (21.9\%) among those aged 18-44 years (from 35.6\% in 1996 to $43.4 \%$ in 2005 ) (Figure).

In 2005, the age-specific prevalence of fair or poor health was significantly lower among persons aged 18-44 years $(43.4 \%, \mathrm{CI}=39.7 \%-47.0 \%)$ than among those aged $45-64$ years $(50.5 \%, \mathrm{CI}=48.9 \%-52.1 \%)$ or $\geq 75$ years $(52.1 \%$, CI $=49.7 \%-54.6 \%)$ (Table). In addition, the age-adjusted prevalence was higher among women compared with men $(51.1 \%$, $\mathrm{CI}=48.9 \%-53.3 \%$ vs $42.6 \%, \mathrm{CI}=39.2 \%-45.9 \%$, respectively); among non-Hispanic blacks and Hispanics compared with non-Hispanic whites $(49.9 \%, \mathrm{CI}=45.8 \%-53.9 \%$ and $59.8 \%, \mathrm{CI}=53.9 \%-65.7 \%$ vs $42.1 \%, \mathrm{CI}=39.9 \%-44.3 \%$, respectively); among persons without health insurance coverage compared with those with coverage $(56.7 \%, \mathrm{CI}=51.5 \%-$ $61.9 \%$ vs $44.5 \%, \mathrm{CI}=42.5 \%-46.6 \%$, respectively); among current smokers compared with nonsmokers $(55.5 \%$,
FIGURE. Prevalence of self-rated fair or poor health among adults with diabetes aged $\geq 18$ years, by age group Behavioral Risk Factor Surveillance System, United States, 1996-2005

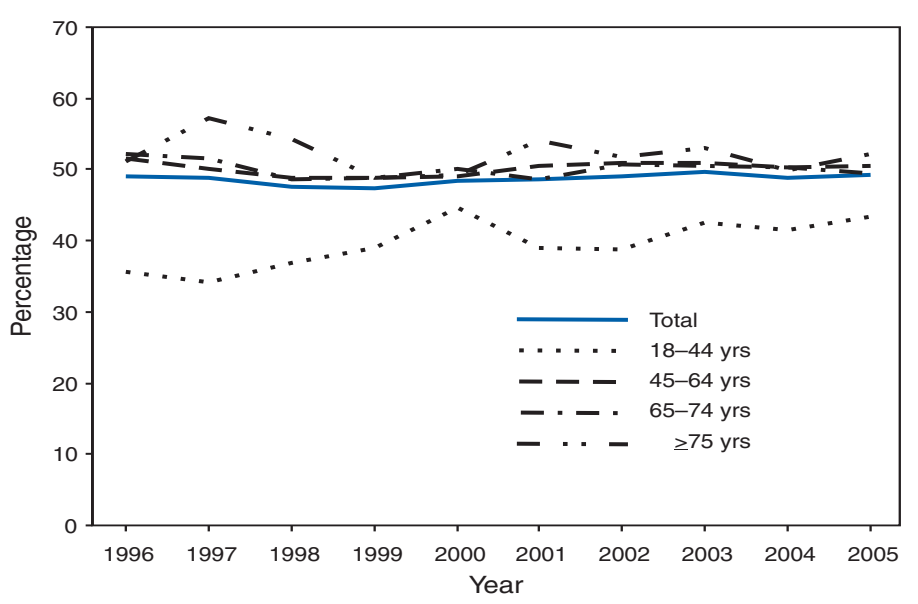

$\mathrm{CI}=51.7 \%-59.3 \%$ vs $44.4 \%, \mathrm{CI}=42.0 \%-46.8 \%$, respectively); and among insulin users compared with those who did not use insulin $(58.5 \%, \mathrm{CI}=54.1 \%-63.0 \%$ vs $43.3 \%$, $\mathrm{CI}=40.6 \%-46.0 \%$, respectively). In addition, as level of education increased, the age-adjusted prevalence decreased. As duration of diabetes increased, prevalence also increased (Table).

In multivariate analyses, the following characteristics were significantly associated with an increased risk for self-rated fair or poor health after adjusting for all other factors: being aged 45-64 years (odds ratio $[\mathrm{OR}]=1.5), 65-74$ years $(\mathrm{OR}=$ $1.4)$, or $\geq 75$ years $(\mathrm{OR}=1.6)$; Hispanic ethnicity $(\mathrm{OR}=1.6)$; current smoking $(\mathrm{OR}=1.7)$; obesity $(\mathrm{OR}=1.4)$; duration of diabetes of $\geq 20$ years $(\mathrm{OR}=1.3)$; and insulin use $(\mathrm{OR}=2.0)$ (Table). In contrast, the following factors were associated with a decreased risk: being a man $(\mathrm{OR}=0.8)$, having a high school education $(\mathrm{OR}=0.5)$ or more than a high school education $(\mathrm{OR}=0.3)$, and having health insurance coverage $(\mathrm{OR}=0.7)$.

Reported by: L Pan, MD, QMukhtar, PhD, SL Geiss, MA, M Rivera, PhD, A Alfaro-Correa, PhD, R Sniegowski, MPH, Div of Diabetes Translation, National Center for Chronic Disease Prevention and Health Promotion, CDC.

Editorial Note: Self-rated health status is a useful indicator of a population's overall well-being because lower ratings of health status have been associated with increased mortality and morbidity (3). Fair or poor health among persons with diabetes is also associated with the presence of diabetesrelated complications such as lower extremity amputation, blindness, kidney failure, and cardiovascular disease (4). The finding that adults with diabetes are more than three times more likely to report fair or poor health than persons without diabetes likely reflects the effects of diabetes and its complica- 
TABLE. Prevalence* of self-rated fair or poor health among adults with diabetes aged $\geq 18$ years, by selected characteristics - Behavioral Risk Factor Surveillance System, United States, 2005

\begin{tabular}{|c|c|c|c|c|}
\hline \multirow[b]{2}{*}{ Characteristic } & \multicolumn{4}{|c|}{ Self-rated fair or poor health } \\
\hline & $(\%)$ & $\left(95 \% \mathrm{Cl}^{\dagger}\right)$ & $\mathrm{OR}^{\S}$ & $(95 \% \mathrm{Cl})$ \\
\hline \multicolumn{5}{|l|}{ Age group (yrs) } \\
\hline $18-44$ & 43.4 & $(39.7-47.0)$ & 一 $^{* *}$ & - \\
\hline $45-64$ & 50.5 & $(48.9-52.1)$ & 1.5 & $(1.2-1.8)$ \\
\hline $65-74$ & 49.4 & $(47.1-51.7)$ & 1.4 & $(1.1-1.7)$ \\
\hline$\geq 75$ & 52.1 & $(49.7-54.6)$ & 1.6 & $(1.3-2.1)$ \\
\hline \multicolumn{5}{|l|}{ Sex } \\
\hline Female & 51.1 & $(48.9-53.3)$ & - & - \\
\hline Male & 42.6 & $(39.2-45.9)$ & 0.8 & $(0.7-0.9)$ \\
\hline \multicolumn{5}{|l|}{ Race/Ethnicity } \\
\hline White, non-Hispanic & 42.1 & (39.9-44.3) & - & - \\
\hline Black, non-Hispanic & 49.9 & $(45.8-53.9)$ & 1.0 & $(0.9-1.2)$ \\
\hline Hispanic & 59.8 & $(53.9-65.7)$ & 1.6 & $(1.3-2.0)$ \\
\hline \multicolumn{5}{|l|}{ Educational levelๆ } \\
\hline Less than high school & 70.4 & $(65.9-74.9)$ & - & - \\
\hline High school & 49.3 & $(46.1-52.5)$ & 0.5 & $(0.4-0.6)$ \\
\hline More than high school & 36.0 & $(33.4-38.6)$ & 0.3 & $(0.2-0.3)$ \\
\hline \multicolumn{5}{|c|}{ Health insurance coverage } \\
\hline No & 56.7 & $(51.5-61.9)$ & - & - \\
\hline Yes & 44.5 & $(42.5-46.6)$ & 0.7 & $(0.6-0.9)$ \\
\hline \multicolumn{5}{|l|}{ Current smoking status } \\
\hline No & 44.4 & $(42.0-46.8)$ & - & - \\
\hline Yes & 55.5 & $(51.7-59.3)$ & 1.7 & $(1.5-2.0)$ \\
\hline \multicolumn{5}{|l|}{ Body mass index ${ }^{\dagger \dagger}$} \\
\hline Normal & 43.4 & $(37.5-49.3)$ & - & - \\
\hline Overweight & 43.6 & $(39.7-47.4)$ & 1.1 & $(0.9-1.3)$ \\
\hline Obese & 49.7 & (47.2-52.2) & 1.4 & $(1.2-1.7)$ \\
\hline \multicolumn{5}{|l|}{ Diabetes duration (yrs) } \\
\hline $0-4$ & 45.0 & $(41.5-48.6)$ & - & - \\
\hline $5-9$ & 41.9 & (37.5-46.3) & 1.0 & $(0.9-1.2)$ \\
\hline $10-19$ & 53.7 & (48.2-59.3) & 1.2 & $(1.0-1.4)$ \\
\hline$\geq 20$ & 48.9 & (43.3-54.5) & 1.3 & $(1.1-1.6)$ \\
\hline \multicolumn{5}{|l|}{ Insulin use } \\
\hline No & 43.3 & $(40.6-46.0)$ & - & - \\
\hline Yes & 58.5 & $(54.1-63.0)$ & 2.0 & $(1.7-2.3)$ \\
\hline \multicolumn{5}{|c|}{$\begin{array}{l}\text { * Age adjusted to the } 2000 \text { U.S. standard adult population, except for the } \\
\text { four age groups, for which crude data are presented. } \\
\text { † Confidence interval. } \\
\text { \& Odds ratio; model includes all variables. } \\
\text { I Significant trend }(p<0.05) \text { among subcategories. } \\
\text { ** Reference group. } \\
\text { †† Body mass index }=\text { weight }(\mathrm{kg}) / \text { height }\left(\mathrm{m}^{2}\right) \text {. Normal = 18.5-24.9; over- } \\
\text { weight }=25.0-29.9 \text {; obese }=\geq 30.0 \text {. }\end{array}$} \\
\hline
\end{tabular}

tions on quality of life. In contrast to older adults, the prevalence of fair or poor health increased during the past decade among young adults with diabetes. Additional research is needed to identify the factors related to this trend.

Consistent with previous studies (4- 6 ), self-rated fair or poor health correlates with certain health risk factors, illness severity, and certain sociodemographic characteristics. Health risk factors such as smoking and obesity are associated with fair or poor health, as are certain indicators of disease severity, such as insulin use and duration of diabetes. Among those with diabetes, subgroups such as older persons, women, Hispanics, persons with less than a high school education, and persons without health insurance coverage are more likely to report fair or poor health. The disparities among these subgroups might result from differences in the prevalence of diabetesrelated complications; access to health-care services; quality of care received; and behavioral, social, or cultural factors. These disparities suggest the need for targeted interventions, such as promoting healthy behaviors through effective smoking cessation and weight-loss programs, improving diabetes management through preventive-care practices, and increasing access to health-care services.

The findings in this report are subject to at least two limitations. First, BRFSS excludes persons who do not have landline telephones, thus the results might not be representative of certain segments of the U.S. population. Second, self-rated health is subjective, and psychosocial factors such as level of social support and beliefs about certain health behaviors can affect how persons respond to questions about self-rated health ( 7 ). However, the retest consistency of respondent self-rated health has been validated $(8)$.

Two of CDC's health protection goals are "live a healthy, productive, and satisfying life" and "live better, longer" (9). CDC provides funding, resources, and technical assistance to 59 diabetes prevention and control programs in the United States. Continued surveillance of the health status of persons with diabetes monitors the well-being of this population and the effectiveness of prevention strategies and provides data for public health agencies that are creating programs to promote population health. Collaboration among health-care systems, health-care providers, policymakers, and other organizations are needed to create interventions to improve the health of persons with diabetes. For example, diabetes education and counseling can improve patients' self-perceived health by enhancing their feelings of self-efficacy (7). The National Diabetes Education Program (NDEP), which is cosponsored by CDC and the National Institutes of Health, educates persons with diabetes about risk factors, raises public awareness of diabetes-related complications, and attempts to improve outcomes of diabetes through partnerships with other sectors of the U.S. health-care system. Additional information about NDEP is available at http://www.ndep.nih.gov.

\section{References}

1. CDC. National diabetes fact sheet: general information and national estimates on diabetes in the United States, 2005. Atlanta, GA: US Department of Health and Human Services, CDC; 2005. Available at http://www.cdc.gov/diabetes/pubs/pdf/ndfs_2005.pdf.

2. US Department of Health and Human Services. Healthy people 2010 (conference ed, in 2 vols). Washington, DC: US Department of Health and Human Services; 2000. Available at http://www.health.gov/ healthypeople. 
3. Hennessy CH, Moriarty DG, Zack MM, Scherr PA, Brackbill R. Measuring health-related quality of life for public health surveillance. Public Health Rep 1994;109:665-72.

4. Rubin R, Peyrot M. Quality of life and diabetes. Diabetes Metab Res Rev 1999;15:205-18.

5. Colsher PL, Wallace RB, Pomrehn PR, et al. Demographic and health characteristics of elderly smokers: results from Established Populations for Epidemiologic Studies of the Elderly. Am J Prev Med 1990;2:61-70.

6. Brown DW, Balluz LS, Giles WH, et al. Diabetes mellitus and healthrelated quality of life among older adults. Findings from the Behavioral Risk Factor Surveillance System (BRFSS). Diabetes Res Clin Pract 2004;65:105-15.

7. Aalto AM, Uutela A, Aro AR. Health related quality of life among insulin-dependent diabetics: disease-related and psychosocial correlates. Patient Educ Couns 1997;30:215-25.

8. Andresen EM, Catlin T, Wyrwich KW, Jackson-Thompson J. Retest reliability of surveillance questions on health related quality of life. J Epidemiol Community Health 2003;57:339-43.

9. CDC. Health protection goals. Atlanta, GA: US Department of Health and Human Services, CDC; 2006. Available at http://www.cdc.gov/ about/goals/Health_Protection_Goals.pdf.

\section{Brief Report}

\section{Hazardous Materials Release Resulting from Home Production of Biodiesel - Colorado, May 2006}

On May 7, 2006, a hazardous materials (HazMat) release occurred in a residential area of Colorado when a homeowner who was processing a tank of homemade biodiesel fuel forgot to turn off the tank's heating element and left for the weekend. The heating element overheated and caused a fire that burned the surrounding shed and equipment (Figure). The shed had contained $>600$ gallons of biodiesel and recycled restaurant cooking oil, smaller amounts of glycerin

FIGURE. Shed debris resulting from home-based biodiesel production fire - Colorado, 2006

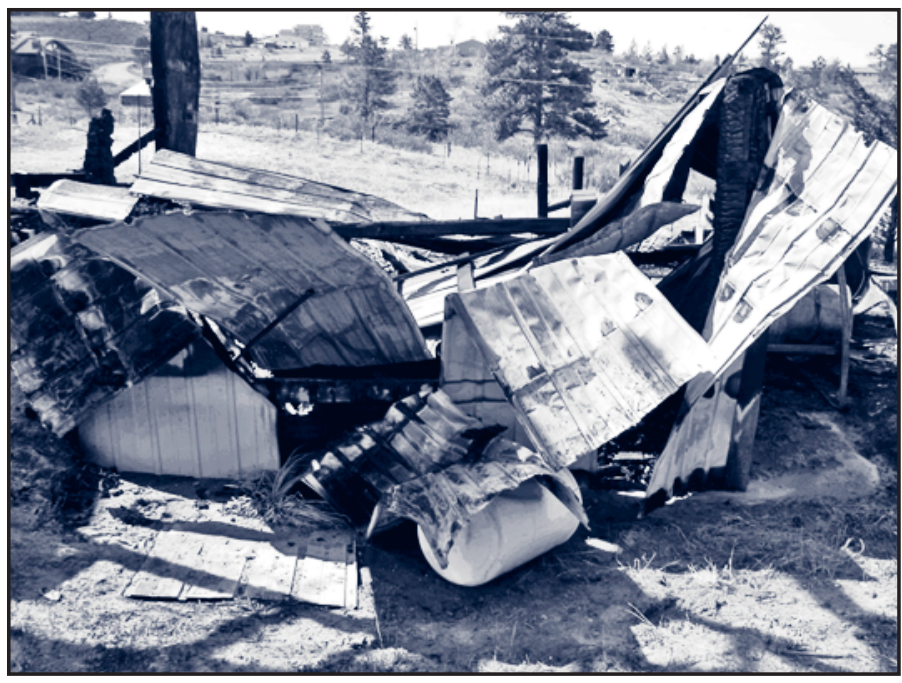

Photo/Kenneth Killip and sodium hydroxide, and 1-gallon containers of sulfuric and phosphoric acid; a mixture of these ingredients seeped into the ground during the fire. A certified HazMat team and the local fire department responded. Investigators found seven 55-gallon barrels of methanol and other hazardous materials outside the shed. No injuries or evacuations occurred. To prevent potential injuries, biodiesel should be purchased from a licensed commercial source.

The recent rise in petroleum prices has caused an increased interest in alternative fuels such as biodiesel (1). Although many alternative fuels exist (e.g., ethanol, hydrogen, and natural gas), biodiesel is used increasingly as a diesel-replacement fuel in the United States because it can be manufactured from readily available ingredients such as vegetable oil, animal fat, or recycled restaurant cooking oil (2). Biodiesel is created through a chemical process involving the reaction of fat or oil with methanol in the presence of a catalyst (e.g., sodium or potassium hydroxide) to produce methyl ester (i.e., biodiesel) and glycerin, a byproduct used in soap and other products $(3,4)$. Biodiesel can be used in vehicles and machinery designed to operate on diesel fuel, such as automobiles with diesel (but not gasoline) engines, fuel and heating-oil boilers, and nonaviation turbines (3).

Biodiesel usually is produced commercially; however, some persons in the United States and elsewhere produce biodiesel in their homes for personal use. Those who produce homemade biodiesel should be aware of the substantial risk for injury. Substances used in biodiesel production can be highly explosive (i.e., methanol) or corrosive (i.e., sodium hydroxide). If improperly handled, these substances can cause severe eye, skin, and upper respiratory irritation; chemical burns; and other serious injuries (5-7). During the preceding 10 years, almost all fires and injuries caused by home production of biodiesel of which the National Biodiesel Board (NBB) is aware were caused by improper handling of methanol during production. NBB is the nonprofit trade association coordinating regulatory, technical, and market development of the fuel as a commercial product. The event described in this report is the first known to NBB involving a heating element in an unintentional fire related to home production of biodiesel.

This HazMat event was reported to the Hazardous Substances Emergency Events Surveillance (HSEES) system operated by the Colorado Department of Health and Environment; HSEES was created by the Agency for Toxic Substances and Disease Registry (ATSDR) (8). This multistate* health department surveillance system tracks morbidity and

\footnotetext{
*Colorado, Florida, Iowa, Louisiana, Michigan, Minnesota, New Jersey, New
} York, North Carolina, Oregon, Texas, Utah, Washington, and Wisconsin. 
mortality resulting from events ${ }^{\dagger}$ involving the release of hazardous substances. However, because reporting HazMat events to HSEES is not mandatory, participating state health departments might not be informed about every event.

Production of homemade biodiesel can be dangerous for persons without appropriate training and equipment. Therefore, this fuel should be purchased from a licensed source.

Reported by: K Killip, Hazardous Materials Response Team, Parker Fire Protection District, Arapaho/Douglas County; C Kelley, Colorado Dept of Health and Environment. S Howell, National Biodiesel Board, Jefferson City, Missouri. DK Horton, MSPH, MOrr, MS, Div of Health Studies, Agency for Toxic Substances and Disease Registry.

${ }^{\dagger}$ An event is defined as a sudden, uncontrolled, or illegal release or threatened release of at least $10 \mathrm{lbs}$ or 1 gallon of a hazardous substance or any amount of a hazardous substance if it is on the mandatory reporting list.

\section{References}

1. National Renewable Energy Laboratory. Survey of the quality and stability of biodiesel and biodiesel blends in the United States in 2004. Golden, CO: US Department of Energy, Office of Energy Efficiency and Renewable Energy; 2005. Available at http://www.biodiesel.org/ resources/reportsdatabase/reports/gen/22051001_gen356.pdf.

2. US Department of Energy. Alternative fuels, biodiesel. Golden, CO: US Department of Energy, Office of Energy Efficiency and Renewable Energy; 2006. Available at http://www.eere.energy.gov/afdc/altfuel/ biodiesel.html.

3. US Department of Energy. Biodiesel handling and use guidelines. DOE/ GO-102006-2288, 2nd ed. Golden, CO: US Department of Energy, Office of Energy Efficiency and Renewable Energy; 2006. Available at http://www.nrel.gov/vehiclesandfuels/npbf/pdfs/40555.pdf.

4. National Biodiesel Board. Biodiesel basics. Jefferson City, MO: National Biodiesel Board; 2006. Available at http://www.biodiesel.org.

5. US Department of Transportation. North American emergency response guidebook. Washington, DC: US Department of Transportation; 2004. Available at http://hazmat.dot.gov/pubs/erg/gydebook.htm.

6. Agency for Toxic Substances and Disease Registry. ToxFAQs ${ }^{\mathrm{TM}}$ for sodium hydroxide. Atlanta, GA: US Department of Health and Human Services, Agency for Toxic Substances and Disease Registry; 2002. Available at http://www.atsdr.cdc.gov/tfacts178.html.

7. National Institute for Occupational Safety and Health. NIOSH pocket guide to chemical hazards. Cincinnati, $\mathrm{OH}$ : US Department of Health and Human Services, CDC, National Institute for Occupational Safety and Health; 2005. Available at http://www.cdc.gov/niosh/npg/npg.html.

8. Agency for Toxic Substances and Disease Registry. Hazardous Substances Emergency Events Surveillance system annual report, 2003. Atlanta, GA: US Department of Health and Human Services, Agency for Toxic Substances and Disease Registry; 2004. Available at http://www.atsdr. cdc.gov/HS/HSEES.

\section{Notice to Readers}

\section{Status Report on CDC Laboratory Animal Care Accreditation}

CDC conducts vital animal research to understand and ultimately prevent viral, mycotic, bacterial, and other diseases that threaten populations worldwide. CDC has a moral and ethical responsibility to humanely care for the animals that contribute to this research.

Since 1967, CDC has participated in and received accreditation from the Association for Assessment and Accreditation of Laboratory Animal Care International (AAALAC) program. This accreditation process is an added safeguard to ensure ethical and humane treatment and care of the animals entrusted to the agency for participation in its research programs.

In late 2005, AAALAC conducted a review of CDC's research programs and laboratories for conducting animal research and noted certain areas in need of improvement, including the policies and procedures of CDC's Institutional Animal Care and Use Committee. AAALAC issued recommendations for raising the quality of animal care at CDC and enhancing worker safety. As a result of the AAALAC findings, CDC's accreditation was placed on probationary status.

In response to this review, CDC conducted it own investigation. Subsequently, during 2006, CDC upgraded its laboratory research facilities, improved the electronic records management system for its animal care program, and hired additional staff members to carry out the oversight and recordkeeping functions required for the animal care and use program. In addition, CDC changed lines of authority and responsibility to ensure impartial and credible oversight, including moving oversight for the animal care and use program to the Office of the Director, putting it on equal standing with oversight for human subjects research, and assigning three veterinarians and two animal caretakers with independent access to the agency's Biosafety Level 4 laboratory.

In late October 2006, a five-member panel from AAALAC conducted a follow-up site visit to CDC's Atlanta campus; the official report is pending. CDC expects a full report from AAALAC in early 2007. Additional information regarding CDC's animal research facilities, practices, and electronic records management systems is available at http://www.cdc. gov/od/science/regs/acup. 


\section{QuickStats}

FROM THE NATIONAL CENTER FOR HEALTH STATISTICS

\section{Prevalence of Overweight* Among Persons Aged 2-19 Years, by Sex - National Health and Nutrition Examination Survey (NHANES), United States, 1999-2000 Through 2003-2004}

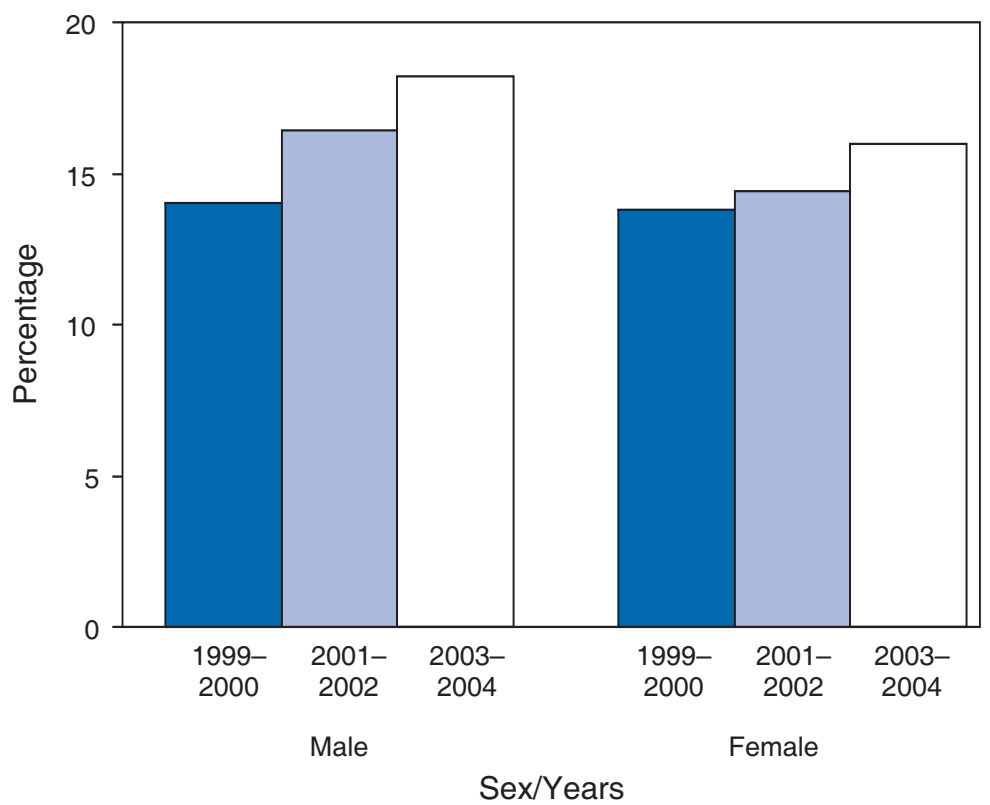

\footnotetext{
* Defined as having a body mass index (weight $[\mathrm{kg}] /$ height $\left[\mathrm{m}^{2}\right]$ ) at or above the 95th percentile for age and sex based on the reference population of the CDC 2000 growth charts (available at http://www.cdc.gov/growthcharts).
}

From 1999-2000 through 2003-2004, the prevalence of overweight among males and females increased from $14.0 \%$ to $18.2 \%$ and from $13.8 \%$ to $16.0 \%$, respectively. By $2003-2004$, approximately 12.5 million persons aged 12-19 years (17.1\%) were overweight. Additional information regarding NHANES is available at http:// www.cdc.gov/nchs/nhanes.htm.

SOURCE: Ogden CL, Carroll MD, Curtin LR, McDowell MA, Tabak CJ, Flegal KM. Prevalence of overweight and obesity in the United States, 1999-2004. JAMA 2006;295:1549-55. 
TABLE I. Provisional cases of infrequently reported notifiable diseases ( $<1,000$ cases reported during the preceding year) - United States, week ending November 11, 2006 (45th Week)*

\begin{tabular}{|c|c|c|c|c|c|c|c|c|c|}
\hline \multirow[b]{2}{*}{ Disease } & \multirow{2}{*}{$\begin{array}{l}\text { Current } \\
\text { week }\end{array}$} & \multirow{2}{*}{$\begin{array}{l}\text { Cum } \\
2006\end{array}$} & \multirow{2}{*}{$\begin{array}{c}\text { 5-year } \\
\text { weekly } \\
\text { average }^{\dagger}\end{array}$} & \multicolumn{5}{|c|}{ Total cases reported for previous years } & \multirow[b]{2}{*}{ States reporting cases during current week (No.) } \\
\hline & & & & 2005 & 2004 & 2003 & 2002 & 2001 & \\
\hline Anthrax & - & 1 & 0 & - & - & - & 2 & 23 & \\
\hline \multicolumn{10}{|l|}{ Botulism: } \\
\hline foodborne & - & 8 & 0 & 19 & 16 & 20 & 28 & 39 & \\
\hline infant & - & 69 & 1 & 90 & 87 & 76 & 69 & 97 & \\
\hline other (wound \& unspecified) & - & 44 & 0 & 33 & 30 & 33 & 21 & 19 & \\
\hline Brucellosis & - & 92 & 3 & 122 & 114 & 104 & 125 & 136 & \\
\hline Chancroid & - & 26 & 1 & 17 & 30 & 54 & 67 & 38 & \\
\hline Cholera & - & 6 & 0 & 8 & 5 & 2 & 2 & 3 & \\
\hline Cyclosporiasis $§$ & 2 & 106 & 2 & 716 & 171 & $7 \overline{5}$ & 156 & 147 & $\mathrm{NC}(2)$ \\
\hline Diphtheria & - & - & 0 & - & - & 1 & 1 & 2 & \\
\hline \multicolumn{10}{|l|}{ Domestic arboviral diseases $\$$, } \\
\hline California serogroup & - & 46 & 2 & 80 & 112 & 108 & 164 & 128 & \\
\hline eastern equine & - & 6 & 0 & 21 & 6 & 14 & 10 & 9 & \\
\hline Powassan & - & 1 & - & 1 & 1 & - & 1 & $\mathrm{~N}$ & \\
\hline St. Louis & - & 7 & 0 & 13 & 12 & 41 & 28 & 79 & \\
\hline western equine & - & - & - & - & - & - & - & - & \\
\hline \multicolumn{10}{|l|}{ Ehrlichiosisई: } \\
\hline human granulocytic & 3 & 332 & 8 & 790 & 537 & 362 & 511 & 261 & NY (3) \\
\hline human monocytic & 7 & 333 & 6 & 521 & 338 & 321 & 216 & 142 & NY (2), NC (4), TN (1) \\
\hline human (other \& unspecified) & 1 & 139 & 1 & 122 & 59 & 44 & 23 & 6 & $\mathrm{NC}(1)$ \\
\hline \multirow{2}{*}{\multicolumn{10}{|c|}{$\begin{array}{l}\text { Haemophilus influenzae, } \\
\text { invasive disease (age }<5 \mathrm{yrs} \text { ): }\end{array}$}} \\
\hline & & & & & & & & & \\
\hline serotype b & - & 9 & 0 & 9 & 19 & 32 & 34 & - & \\
\hline nonserotype b & 2 & 72 & 3 & 135 & 135 & 117 & 144 & - & $\mathrm{CA}(2)$ \\
\hline unknown serotype & 2 & 166 & 2 & 217 & 177 & 227 & 153 & - & PA (1), FL (1) \\
\hline Hansen disease ${ }^{\S}$ & 1 & 63 & 2 & 88 & 105 & 95 & 96 & 79 & $\mathrm{CA}(1)$ \\
\hline Hantavirus pulmonary syndrome ${ }^{\S}$ & - & 26 & 0 & 29 & 24 & 26 & 19 & 8 & \\
\hline Hemolytic uremic syndrome, postdiarrheal ${ }^{\S}$ & - & 216 & 4 & 221 & 200 & 178 & 216 & 202 & \\
\hline Hepatitis C viral, acute & 4 & 649 & 29 & 751 & 713 & 1,102 & 1,835 & 3,976 & ME (1), WV (1), FL (1), CA (1) \\
\hline HIV infection, pediatric (age $<13$ yrs) $)^{\text {s.t† }}$ & - & 52 & 6 & 380 & 436 & 504 & 420 & 543 & \\
\hline Influenza-associated pediatric mortality $\S, \S \S$ & - & 40 & 0 & 45 & - & $\mathrm{N}$ & $\mathrm{N}$ & $\mathrm{N}$ & \\
\hline Listeriosis & 10 & 615 & 15 & 892 & 753 & 696 & 665 & 613 & NY (1), PA (1), OH (3), IN (1), NC (1), TX (1), CA (2) \\
\hline Measles & - & 44 & 1 & 66 & 37 & 56 & 44 & 116 & \\
\hline \multicolumn{10}{|l|}{ Meningococcal disease, invasive ${ }^{\star * \star}$ : } \\
\hline$A, C, Y, \& W-135$ & 2 & 168 & 3 & 297 & - & - & - & - & $\mathrm{OH}(1), \mathrm{OK}(1)$ \\
\hline serogroup $B$ & 1 & 106 & 2 & 157 & - & - & - & - & OK (1) \\
\hline other serogroup & 1 & 16 & 0 & 27 & - & - & - & - & OK (1) \\
\hline Mumps & 9 & 5,980 & 5 & 314 & 258 & 231 & 270 & 266 & $\mathrm{NY}(1), \mathrm{OH}(2), \mathrm{MO}(1), \mathrm{KS}(5)$ \\
\hline Plague & - & 16 & 0 & 8 & 3 & 1 & 2 & 2 & \\
\hline Poliomyelitis, paralytic & - & - & - & 1 & - & - & - & - & \\
\hline Psittacosis§ & - & 18 & 1 & 19 & 12 & 12 & 18 & 25 & \\
\hline$Q$ fever $\$$ & 1 & 130 & 1 & 139 & 70 & 71 & 61 & 26 & $\mathrm{CA}(1)$ \\
\hline Rabies, human & - & 1 & - & 2 & 7 & 2 & 3 & 1 & \\
\hline Rubella & - & 9 & - & 11 & 10 & 7 & 18 & 23 & \\
\hline Rubella, congenital syndrome & - & 1 & - & 1 & - & 1 & 1 & 3 & \\
\hline SARS-CoVs.ttt & - & - & - & - & - & 8 & $\mathrm{~N}$ & $\mathrm{~N}$ & \\
\hline Smallpox & - & - & - & - & - & - & - & - & \\
\hline Streptococcal toxic-shock syndrome $§$ & 1 & 84 & 1 & 129 & 132 & 161 & 118 & 77 & $\mathrm{OH}(1)$ \\
\hline \multicolumn{10}{|l|}{ Streptococcus pneumoniae, $\$$} \\
\hline invasive disease (age < 5 yrs) & 17 & 939 & 16 & 1,257 & 1,162 & 845 & 513 & 498 & $\mathrm{NY}(3), \mathrm{OH}(7), \mathrm{MI}(1), \mathrm{MD}(5), \mathrm{CO}(1)$ \\
\hline Syphilis, congenital (age <1 yr) & - & 234 & 8 & 361 & 353 & 413 & 412 & 441 & \\
\hline Tetanus & 1 & 19 & 0 & 27 & 34 & 20 & 25 & 37 & $\mathrm{FL}(1)$ \\
\hline Toxic-shock syndrome (other than streptococcal)§ & )$^{\S} 1$ & 83 & 2 & 96 & 95 & 133 & 109 & 127 & $\mathrm{KS}(1)$ \\
\hline Trichinellosis & - & 11 & 0 & 19 & 5 & 6 & 14 & 22 & \\
\hline Tularemia & - & 77 & 2 & 154 & 134 & 129 & 90 & 129 & \\
\hline Typhoid fever & 1 & 238 & 5 & 324 & 322 & 356 & 321 & 368 & $\mathrm{CA}(1)$ \\
\hline Vancomycin-intermediate Staphylococcus aureus & $s^{\S}-$ & 3 & 0 & 2 & - & $\mathrm{N}$ & $\mathrm{N}$ & $\mathrm{N}$ & \\
\hline Vancomycin-resistant Staphylococcus aureus ${ }^{\S}$ & - & - & - & 3 & 1 & $\mathrm{~N}$ & $\mathrm{~N}$ & $\mathrm{~N}$ & \\
\hline Yellow fever & - & - & - & - & - & - & 1 & - & \\
\hline
\end{tabular}

-: No reported cases. $\quad \mathrm{N}$ : Not notifiable. Cum: Cumulative year-to-date counts.

* Incidence data for reporting year 2006 are provisional, whereas data for 2001, 2002, 2003, 2004, and 2005 are finalized.

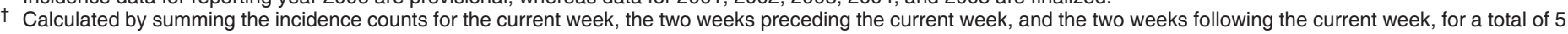
preceding years. Additional information is available at http://www.cdc.gov/epo/dphsi/phs/files/5yearweeklyaverage.pdf.

$\S$ Not notifiable in all states.

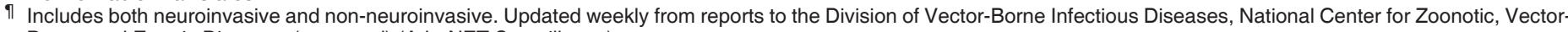
Borne, and Enteric Diseases (proposed) (ArboNET Surveillance).

** Data for $H$. influenzae (all ages, all serotypes) are available in Table II.

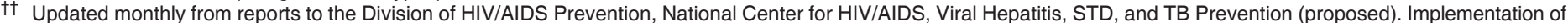

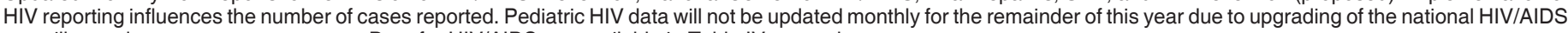
surveillance data management system. Data for HIV/AIDS are available in Table IV quarterly.

$\S \S$ Updated weekly from reports to the Influenza Division, National Center for Immunization and Respiratory Diseases (proposed).

ๆी No measles cases were reported for the current week.

*** Data for meningococcal disease (all serogroups and unknown serogroups) are available in Table II.

t†t Updated weekly from reports to the Division of Viral and Rickettsial Diseases, National Center for Zoonotic, Vector-Borne, and Enteric Diseases (proposed). 
TABLE II. Provisional cases of selected notifiable diseases, United States, weeks ending November 11, 2006, and November 12, 2005 (45th Week)*

\begin{tabular}{|c|c|c|c|c|c|c|c|c|c|c|c|c|c|c|c|}
\hline \multirow[b]{3}{*}{ Reporting area } & \multicolumn{5}{|c|}{ Chlamydia $^{\dagger}$} & \multicolumn{5}{|c|}{ Coccidioidomycosis } & \multicolumn{5}{|c|}{ Cryptosporidiosis } \\
\hline & \multirow{2}{*}{$\begin{array}{c}\text { Current } \\
\text { week }\end{array}$} & \multicolumn{2}{|c|}{$\begin{array}{c}\text { Previous } \\
52 \text { weeks } \\
\end{array}$} & \multirow{2}{*}{$\begin{array}{l}\text { Cum } \\
2006\end{array}$} & \multirow{2}{*}{$\begin{array}{l}\text { Cum } \\
2005\end{array}$} & \multirow{2}{*}{$\begin{array}{c}\text { Current } \\
\text { week }\end{array}$} & \multicolumn{2}{|c|}{$\begin{array}{l}\text { Previous } \\
52 \text { weeks }\end{array}$} & \multirow{2}{*}{$\begin{array}{l}\text { Cum } \\
2006\end{array}$} & \multirow{2}{*}{$\begin{array}{l}\text { Cum } \\
2005\end{array}$} & \multirow{2}{*}{$\begin{array}{c}\text { Current } \\
\text { week }\end{array}$} & \multicolumn{2}{|c|}{$\begin{array}{l}\text { Previous } \\
52 \text { weeks } \\
\end{array}$} & \multirow{2}{*}{$\begin{array}{l}\text { Cum } \\
2006\end{array}$} & \multirow{2}{*}{$\begin{array}{c}\text { Cum } \\
2005\end{array}$} \\
\hline & & Med & Max & & & & Med & Max & & & & $\overline{\text { Med }}$ & Max & & \\
\hline United States & 10,236 & 19,286 & 35,170 & 826,278 & 828,989 & 131 & 149 & 1,643 & 6,964 & 3,886 & 48 & 73 & 594 & 4,442 & 6,802 \\
\hline $\begin{array}{l}\text { New England } \\
\text { Connecticut } \\
\text { Maine§ } \\
\text { Massachusetts } \\
\text { New Hampshire } \\
\text { Rhode Island } \\
\text { Vermont }{ }^{\S}\end{array}$ & $\begin{array}{r}764 \\
171 \\
35 \\
429 \\
46 \\
83 \\
-\end{array}$ & $\begin{array}{r}635 \\
178 \\
43 \\
296 \\
38 \\
61 \\
18\end{array}$ & $\begin{array}{r}1,550 \\
1,214 \\
67 \\
608 \\
65 \\
107 \\
43\end{array}$ & $\begin{array}{r}28,922 \\
8,385 \\
1,951 \\
13,311 \\
1,725 \\
2,634 \\
916\end{array}$ & $\begin{array}{r}27,556 \\
7,936 \\
1,950 \\
12,393 \\
1,609 \\
2,838 \\
830\end{array}$ & $\begin{array}{l}\bar{N} \\
\mathrm{~N} \\
- \\
- \\
\mathrm{N}\end{array}$ & $\begin{array}{l}0 \\
0 \\
0 \\
0 \\
0 \\
0 \\
0\end{array}$ & $\begin{array}{l}0 \\
0 \\
0 \\
0 \\
0 \\
0 \\
0\end{array}$ & $\begin{array}{l}\bar{N} \\
N \\
- \\
- \\
N\end{array}$ & $\begin{array}{l}\bar{N} \\
\mathrm{~N} \\
- \\
-\end{array}$ & $\begin{array}{l}- \\
- \\
- \\
-\end{array}$ & $\begin{array}{l}4 \\
0 \\
0 \\
1 \\
1 \\
0 \\
0\end{array}$ & $\begin{array}{r}35 \\
32 \\
4 \\
14 \\
5 \\
6 \\
5\end{array}$ & $\begin{array}{r}257 \\
32 \\
34 \\
88 \\
43 \\
14 \\
46\end{array}$ & $\begin{array}{r}328 \\
77 \\
27 \\
142 \\
34 \\
13 \\
35\end{array}$ \\
\hline $\begin{array}{l}\text { Mid. Atlantic } \\
\text { New Jersey } \\
\text { New York (Upstate) } \\
\text { New York City } \\
\text { Pennsylvania }\end{array}$ & $\begin{array}{r}986 \\
58 \\
244 \\
427 \\
257\end{array}$ & $\begin{array}{r}2,397 \\
363 \\
499 \\
740 \\
757\end{array}$ & $\begin{array}{r}3,696 \\
497 \\
1,727 \\
1,567 \\
1,104\end{array}$ & $\begin{array}{r}104,335 \\
15,482 \\
20,814 \\
33,086 \\
34,953\end{array}$ & $\begin{array}{r}102,493 \\
16,668 \\
20,410 \\
33,371 \\
32,044\end{array}$ & $\begin{array}{l}- \\
N \\
N \\
N \\
N\end{array}$ & $\begin{array}{l}0 \\
0 \\
0 \\
0 \\
0\end{array}$ & $\begin{array}{l}0 \\
0 \\
0 \\
0 \\
0\end{array}$ & $\begin{array}{l}- \\
N \\
N \\
N \\
N\end{array}$ & $\begin{array}{l}-\bar{N} \\
N \\
N \\
N\end{array}$ & $\begin{array}{l}\frac{3}{3} \\
- \\
-\end{array}$ & $\begin{array}{r}11 \\
0 \\
3 \\
2 \\
4\end{array}$ & $\begin{array}{r}444 \\
3 \\
441 \\
7 \\
17\end{array}$ & $\begin{array}{r}499 \\
11 \\
153 \\
88 \\
247\end{array}$ & $\begin{array}{r}2,866 \\
56 \\
2,420 \\
140 \\
250\end{array}$ \\
\hline $\begin{array}{l}\text { E.N. Central } \\
\text { Illinois } \\
\text { Indiana } \\
\text { Michigan } \\
\text { Ohio } \\
\text { Wisconsin }\end{array}$ & $\begin{array}{r}962 \\
348 \\
288 \\
224 \\
18 \\
84\end{array}$ & $\begin{array}{r}3,126 \\
978 \\
390 \\
661 \\
637 \\
391\end{array}$ & $\begin{array}{r}12,578 \\
1,695 \\
510 \\
9,888 \\
1,430 \\
531\end{array}$ & $\begin{array}{r}135,858 \\
45,515 \\
16,820 \\
29,863 \\
26,753 \\
16,907\end{array}$ & $\begin{array}{r}140,573 \\
43,920 \\
17,390 \\
23,507 \\
38,045 \\
17,711\end{array}$ & $\begin{array}{l}- \\
\frac{N}{N} \\
N\end{array}$ & $\begin{array}{l}1 \\
0 \\
0 \\
0 \\
0 \\
0\end{array}$ & $\begin{array}{l}3 \\
0 \\
0 \\
3 \\
2 \\
0\end{array}$ & $\begin{array}{r}41 \\
\mathrm{~N} \\
35 \\
6 \\
\mathrm{~N}\end{array}$ & $\begin{array}{l}\frac{11}{\mathrm{~N}} \\
\frac{11}{\mathrm{~N}}\end{array}$ & $\begin{array}{l}\frac{9}{3} \\
\frac{6}{6} \\
-\end{array}$ & $\begin{array}{r}16 \\
2 \\
1 \\
2 \\
5 \\
5\end{array}$ & $\begin{array}{r}105 \\
18 \\
18 \\
8 \\
33 \\
53\end{array}$ & $\begin{array}{r}1,112 \\
139 \\
88 \\
123 \\
327 \\
435\end{array}$ & $\begin{array}{r}1,538 \\
150 \\
77 \\
100 \\
737 \\
474\end{array}$ \\
\hline $\begin{array}{l}\text { W.N. Central } \\
\text { lowa } \\
\text { Kansas } \\
\text { Minnesota } \\
\text { Missouri } \\
\text { Nebraska } \\
\text { North Dakota } \\
\text { South Dakota }\end{array}$ & $\begin{array}{r}231 \\
\overline{120} \\
- \\
\overline{103} \\
8 \\
-\end{array}$ & $\begin{array}{r}1,157 \\
157 \\
150 \\
231 \\
437 \\
96 \\
34 \\
51\end{array}$ & $\begin{array}{r}1,456 \\
225 \\
269 \\
347 \\
610 \\
176 \\
58 \\
116\end{array}$ & $\begin{array}{r}50,538 \\
7,019 \\
6,200 \\
9,631 \\
19,355 \\
4,664 \\
1,446 \\
2,223\end{array}$ & $\begin{array}{r}51,147 \\
6,347 \\
6,400 \\
10,709 \\
19,450 \\
4,425 \\
1,430 \\
2,386\end{array}$ & $\begin{array}{l}\bar{N} \\
\mathrm{~N} \\
- \\
\mathrm{N} \\
\mathrm{N} \\
\mathrm{N}\end{array}$ & $\begin{array}{l}0 \\
0 \\
0 \\
0 \\
0 \\
0 \\
0 \\
0\end{array}$ & $\begin{array}{r}12 \\
0 \\
0 \\
12 \\
1 \\
0 \\
0 \\
0\end{array}$ & $\begin{array}{l}1 \\
N \\
N \\
1 \\
N \\
N \\
N\end{array}$ & $\begin{array}{l}4 \\
N \\
N \\
3 \\
1 \\
N \\
N \\
N\end{array}$ & $\begin{array}{l}6 \\
1 \\
3 \\
1 \\
1 \\
- \\
-\end{array}$ & $\begin{array}{r}11 \\
1 \\
1 \\
2 \\
2 \\
1 \\
0 \\
1\end{array}$ & $\begin{array}{r}75 \\
28 \\
8 \\
22 \\
18 \\
16 \\
4 \\
7\end{array}$ & $\begin{array}{r}770 \\
165 \\
76 \\
204 \\
161 \\
87 \\
9 \\
68\end{array}$ & $\begin{array}{r}576 \\
119 \\
34 \\
126 \\
241 \\
26 \\
1 \\
29\end{array}$ \\
\hline $\begin{array}{l}\text { S. Atlantic } \\
\text { Delaware } \\
\text { District of Columbia } \\
\text { Florida } \\
\text { Georgia } \\
\text { Maryland } \\
\text { North Carolina } \\
\text { South Carolina } \\
\text { Virginia }^{\S} \\
\text { West Virginia }\end{array}$ & $\begin{array}{r}2,164 \\
37 \\
52 \\
643 \\
8 \\
244 \\
549 \\
254 \\
373 \\
4\end{array}$ & $\begin{array}{r}3,676 \\
68 \\
52 \\
957 \\
661 \\
328 \\
613 \\
318 \\
430 \\
57\end{array}$ & $\begin{array}{r}4,938 \\
92 \\
138 \\
1,156 \\
2,142 \\
468 \\
1,772 \\
1,452 \\
840 \\
226\end{array}$ & $\begin{array}{r}159,388 \\
3,089 \\
2,302 \\
42,219 \\
27,249 \\
14,998 \\
29,265 \\
16,794 \\
20,843 \\
2,629\end{array}$ & $\begin{array}{r}152,499 \\
2,946 \\
3,286 \\
37,232 \\
27,407 \\
16,087 \\
27,300 \\
15,902 \\
20,015 \\
2,324\end{array}$ & $\begin{array}{l}\bar{N} \\
\bar{N} \\
- \\
\mathrm{N} \\
\mathrm{N} \\
\mathrm{N} \\
\mathrm{N}\end{array}$ & $\begin{array}{l}0 \\
0 \\
0 \\
0 \\
0 \\
0 \\
0 \\
0 \\
0 \\
0\end{array}$ & $\begin{array}{l}1 \\
0 \\
0 \\
0 \\
0 \\
1 \\
0 \\
0 \\
0 \\
0\end{array}$ & $\begin{array}{l}3 \\
\mathrm{~N} \\
\mathrm{~N} \\
\\
3 \\
\mathrm{~N} \\
\mathrm{~N} \\
\mathrm{~N} \\
\mathrm{~N}\end{array}$ & $\begin{array}{l}2 \\
\mathrm{~N} \\
\mathrm{~N} \\
- \\
2 \\
\mathrm{~N} \\
\mathrm{~N} \\
\mathrm{~N} \\
\mathrm{~N}\end{array}$ & $\begin{array}{l}23 \\
\overline{-} \\
\frac{19}{-} \\
\frac{4}{-} \\
-\end{array}$ & $\begin{array}{r}15 \\
0 \\
0 \\
6 \\
4 \\
0 \\
1 \\
1 \\
1 \\
0\end{array}$ & $\begin{array}{r}67 \\
3 \\
2 \\
32 \\
12 \\
3 \\
11 \\
13 \\
6 \\
3\end{array}$ & $\begin{array}{r}995 \\
13 \\
13 \\
481 \\
210 \\
15 \\
90 \\
119 \\
45 \\
9\end{array}$ & $\begin{array}{r}650 \\
6 \\
13 \\
303 \\
127 \\
29 \\
77 \\
21 \\
61 \\
13\end{array}$ \\
\hline $\begin{array}{l}\text { E.S. Central } \\
\text { Alabama } \\
\text { Kentucky } \\
\text { Mississippi } \\
\text { Tennessee }^{\S}\end{array}$ & $\begin{array}{r}1,188 \\
23 \\
358 \\
324 \\
483\end{array}$ & $\begin{array}{r}1,391 \\
406 \\
148 \\
363 \\
511\end{array}$ & $\begin{array}{r}1,947 \\
756 \\
402 \\
807 \\
609\end{array}$ & $\begin{array}{r}63,665 \\
17,936 \\
7,202 \\
16,324 \\
22,203\end{array}$ & $\begin{array}{r}60,299 \\
13,988 \\
7,635 \\
18,424 \\
20,252\end{array}$ & $\begin{array}{l}- \\
\mathrm{N} \\
\frac{\mathrm{N}}{\mathrm{N}}\end{array}$ & $\begin{array}{l}0 \\
0 \\
0 \\
0 \\
0\end{array}$ & $\begin{array}{l}0 \\
0 \\
0 \\
0 \\
0\end{array}$ & $\begin{array}{l}\bar{N} \\
\frac{N}{N}\end{array}$ & $\begin{array}{l}\bar{N} \\
\frac{N}{N}\end{array}$ & $\begin{array}{r}2 \\
2 \\
- \\
- \\
-\end{array}$ & $\begin{array}{l}3 \\
1 \\
1 \\
0 \\
0\end{array}$ & $\begin{array}{r}12 \\
10 \\
8 \\
3 \\
5\end{array}$ & $\begin{array}{r}158 \\
70 \\
35 \\
16 \\
37\end{array}$ & $\begin{array}{r}204 \\
23 \\
138 \\
2 \\
41\end{array}$ \\
\hline $\begin{array}{l}\text { W.S. Central } \\
\text { Arkansas } \\
\text { Louisiana } \\
\text { Oklahoma } \\
\text { Texas }\end{array}$ & $\begin{array}{r}1,239 \\
137 \\
74 \\
358 \\
670\end{array}$ & $\begin{array}{r}2,189 \\
155 \\
254 \\
220 \\
1,458\end{array}$ & $\begin{array}{r}3,605 \\
335 \\
608 \\
2,159 \\
1,904\end{array}$ & $\begin{array}{r}95,349 \\
7,174 \\
11,739 \\
10,895 \\
65,541\end{array}$ & $\begin{array}{r}96,125 \\
7,518 \\
14,881 \\
10,279 \\
63,447\end{array}$ & $\begin{array}{c}- \\
\bar{N} \\
\mathrm{~N}\end{array}$ & $\begin{array}{l}0 \\
0 \\
0 \\
0 \\
0\end{array}$ & $\begin{array}{l}1 \\
0 \\
1 \\
0 \\
0\end{array}$ & $\begin{array}{c}1 \\
1 \\
N \\
N\end{array}$ & $\begin{array}{l}- \\
\mathrm{N} \\
\mathrm{N} \\
\mathrm{N}\end{array}$ & $\begin{array}{r}3 \\
1 \\
2 \\
-\end{array}$ & $\begin{array}{l}3 \\
0 \\
0 \\
0 \\
2\end{array}$ & $\begin{array}{r}35 \\
2 \\
9 \\
4 \\
26\end{array}$ & $\begin{array}{r}241 \\
20 \\
54 \\
37 \\
130\end{array}$ & $\begin{array}{r}214 \\
5 \\
78 \\
40 \\
91\end{array}$ \\
\hline $\begin{array}{l}\text { Mountain } \\
\text { Arizona } \\
\text { Colorado } \\
\text { Idaho§ }^{\S} \\
\text { Montana }^{\S} \\
\text { Nevada }^{\S} \\
\text { New Mexico§ } \\
\text { Utah } \\
\text { Wyoming }\end{array}$ & $\begin{array}{r}671 \\
347 \\
49 \\
\overline{14} \\
160 \\
- \\
101 \\
-\end{array}$ & $\begin{array}{r}1,028 \\
368 \\
144 \\
49 \\
43 \\
85 \\
179 \\
94 \\
27\end{array}$ & $\begin{array}{r}1,839 \\
881 \\
482 \\
191 \\
195 \\
432 \\
339 \\
173 \\
54\end{array}$ & $\begin{array}{r}44,277 \\
16,529 \\
5,199 \\
2,333 \\
2,189 \\
4,420 \\
8,126 \\
4,344 \\
1,137\end{array}$ & $\begin{array}{r}54,091 \\
18,316 \\
13,213 \\
2,282 \\
2,019 \\
6,134 \\
7,206 \\
3,928 \\
993\end{array}$ & $\begin{array}{r}10 \\
10 \\
N \\
N \\
N \\
- \\
- \\
-\end{array}$ & $\begin{array}{r}112 \\
108 \\
0 \\
0 \\
0 \\
1 \\
0 \\
1 \\
0\end{array}$ & $\begin{array}{r}452 \\
448 \\
0 \\
0 \\
0 \\
4 \\
3 \\
3 \\
2\end{array}$ & $\begin{array}{r}4,760 \\
4,644 \\
N \\
N \\
N \\
52 \\
13 \\
49 \\
2\end{array}$ & $\begin{array}{r}2,533 \\
2,438 \\
N \\
N \\
N \\
57 \\
17 \\
18 \\
3\end{array}$ & $\begin{array}{l}2 \\
2 \\
- \\
- \\
- \\
-\end{array}$ & $\begin{array}{l}3 \\
0 \\
1 \\
0 \\
1 \\
0 \\
0 \\
0 \\
0\end{array}$ & $\begin{array}{r}39 \\
3 \\
7 \\
5 \\
26 \\
1 \\
5 \\
3 \\
11\end{array}$ & $\begin{array}{r}343 \\
24 \\
64 \\
35 \\
127 \\
9 \\
25 \\
16 \\
43\end{array}$ & $\begin{array}{r}124 \\
9 \\
45 \\
14 \\
16 \\
11 \\
15 \\
11 \\
3\end{array}$ \\
\hline $\begin{array}{l}\text { Pacific } \\
\text { Alaska } \\
\text { California } \\
\text { Hawaii } \\
\text { Oregon } \\
\text { Washington }\end{array}$ & $\begin{array}{r}2,031 \\
132 \\
1,292 \\
- \\
119 \\
488\end{array}$ & $\begin{array}{r}3,323 \\
81 \\
2,578 \\
102 \\
170 \\
340\end{array}$ & $\begin{array}{r}5,079 \\
152 \\
4,231 \\
135 \\
315 \\
604\end{array}$ & $\begin{array}{r}143,946 \\
3,617 \\
112,822 \\
4,479 \\
7,638 \\
15,390\end{array}$ & $\begin{array}{r}144,206 \\
3,680 \\
111,921 \\
4,795 \\
7,704 \\
16,106\end{array}$ & $\begin{array}{r}121 \\
121 \\
N \\
N \\
N\end{array}$ & $\begin{array}{r}43 \\
0 \\
43 \\
0 \\
0 \\
0\end{array}$ & $\begin{array}{r}1,179 \\
0 \\
1,179 \\
0 \\
0 \\
0\end{array}$ & $\begin{array}{r}2,158 \\
\frac{-}{2,158} \\
N \\
N \\
N\end{array}$ & $\begin{array}{r}1,336 \\
1,336 \\
N \\
N \\
N\end{array}$ & $\begin{array}{l}- \\
- \\
- \\
-\end{array}$ & $\begin{array}{l}1 \\
0 \\
0 \\
0 \\
1 \\
0\end{array}$ & $\begin{array}{r}52 \\
1 \\
14 \\
1 \\
6 \\
38\end{array}$ & $\begin{array}{r}67 \\
4 \\
4 \\
59 \\
\end{array}$ & $\begin{array}{r}302 \\
3 \\
176 \\
1 \\
66 \\
56\end{array}$ \\
\hline $\begin{array}{l}\text { American Samoa } \\
\text { C.N.M.I. } \\
\text { Guam } \\
\text { Puerto Rico } \\
\text { U.S. Virgin Islands }\end{array}$ & $\frac{U}{U} \frac{U}{163}$ & $\begin{array}{r}0 \\
0 \\
17 \\
77 \\
5\end{array}$ & $\begin{array}{r}46 \\
0 \\
27 \\
187 \\
16\end{array}$ & 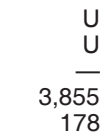 & $\begin{array}{r}U \\
U \\
734 \\
3,576 \\
196\end{array}$ & $\frac{U}{U} \frac{\mathrm{U}}{\mathrm{N}}$ & $\begin{array}{l}0 \\
0 \\
0 \\
0 \\
0\end{array}$ & $\begin{array}{l}0 \\
0 \\
0 \\
0 \\
0\end{array}$ & $\frac{U}{U}$ & $\frac{U}{U}$ & $\frac{U}{U} \frac{U}{-}$ & $\begin{array}{l}0 \\
0 \\
0 \\
0 \\
0\end{array}$ & $\begin{array}{l}0 \\
0 \\
0 \\
0 \\
0\end{array}$ & $\frac{U}{U}$ & $\frac{U}{U}$ \\
\hline
\end{tabular}

C.N.M.I.: Commonwealth of Northern Mariana Islands.

U: Unavailable. ․: No reported cases. N: Not notifiable. Cum: Cumulative year-to-date counts.

Med: Median.

Max: Maximum.

.

Chlamydia refers to genital infections caused by Chlamydia trachomatis.

$\S$ Contains data reported through the National Electronic Disease Surveillance System (NEDSS). 
TABLE II. (Continued) Provisional cases of selected notifiable diseases, United States, weeks ending November 11, 2006, and November 12, 2005 (45th Week)*

\begin{tabular}{|c|c|c|c|c|c|c|c|c|c|c|c|c|c|c|c|}
\hline \multirow[b]{3}{*}{ Reporting area } & \multicolumn{5}{|c|}{ Giardiasis } & \multicolumn{5}{|c|}{ Gonorrhea } & \multicolumn{5}{|c|}{$\begin{array}{l}\text { Haemophilus influenzae, invasive } \\
\text { All ages, all serotypes }\end{array}$} \\
\hline & \multirow{2}{*}{$\begin{array}{c}\text { Current } \\
\text { week }\end{array}$} & \multicolumn{2}{|c|}{$\begin{array}{l}\text { Previous } \\
52 \text { weeks }\end{array}$} & \multirow{2}{*}{$\begin{array}{l}\text { Cum } \\
2006\end{array}$} & \multirow{2}{*}{$\begin{array}{l}\text { Cum } \\
2005\end{array}$} & \multirow{2}{*}{$\begin{array}{c}\text { Current } \\
\text { week }\end{array}$} & \multicolumn{2}{|c|}{$\begin{array}{l}\text { Previous } \\
52 \text { weeks } \\
\end{array}$} & \multirow{2}{*}{$\begin{array}{l}\text { Cum } \\
2006\end{array}$} & \multirow{2}{*}{$\begin{array}{l}\text { Cum } \\
2005\end{array}$} & \multirow{2}{*}{$\begin{array}{c}\text { Current } \\
\text { week }\end{array}$} & \multicolumn{2}{|c|}{$\begin{array}{l}\text { Previous } \\
52 \text { weeks }\end{array}$} & \multirow{2}{*}{$\begin{array}{l}\text { Cum } \\
2006\end{array}$} & \multirow{2}{*}{$\begin{array}{l}\text { Cum } \\
2005 \\
\end{array}$} \\
\hline & & Med & Max & & & & Med & Max & & & & Med & Max & & \\
\hline United States & 183 & 319 & 1,029 & 14,647 & 16,768 & 3,524 & 6,520 & 14,136 & 287,635 & 285,618 & 13 & 40 & 142 & 1,698 & 1,937 \\
\hline $\begin{array}{l}\text { New England } \\
\text { Connecticut } \\
\text { Maine }^{\dagger} \\
\text { Massachusetts } \\
\text { New Hampshire } \\
\text { Rhode Island } \\
\text { Vermont }^{\dagger}\end{array}$ & $\begin{array}{l}\frac{4}{4} \\
- \\
- \\
-\end{array}$ & $\begin{array}{r}23 \\
0 \\
2 \\
9 \\
0 \\
1 \\
3\end{array}$ & $\begin{array}{r}75 \\
37 \\
13 \\
18 \\
9 \\
25 \\
12\end{array}$ & $\begin{array}{r}1,054 \\
253 \\
155 \\
357 \\
26 \\
100 \\
163\end{array}$ & $\begin{array}{r}1,490 \\
310 \\
187 \\
667 \\
54 \\
107 \\
165\end{array}$ & $\begin{array}{r}120 \\
40 \\
1 \\
61 \\
18 \\
\end{array}$ & $\begin{array}{r}109 \\
43 \\
2 \\
46 \\
3 \\
9 \\
1\end{array}$ & $\begin{array}{r}288 \\
241 \\
8 \\
86 \\
9 \\
19 \\
4\end{array}$ & $\begin{array}{r}4,876 \\
1,984 \\
114 \\
2,122 \\
166 \\
432 \\
58\end{array}$ & $\begin{array}{r}4,880 \\
2,044 \\
118 \\
2,142 \\
149 \\
376 \\
51\end{array}$ & $\begin{array}{l}1 \\
- \\
- \\
- \\
-\end{array}$ & $\begin{array}{l}2 \\
0 \\
0 \\
1 \\
0 \\
0 \\
0\end{array}$ & $\begin{array}{r}19 \\
9 \\
4 \\
7 \\
2 \\
7 \\
2\end{array}$ & $\begin{array}{r}133 \\
42 \\
17 \\
52 \\
9 \\
4 \\
9\end{array}$ & $\begin{array}{r}146 \\
43 \\
8 \\
71 \\
8 \\
7 \\
9\end{array}$ \\
\hline $\begin{array}{l}\text { Mid. Atlantic } \\
\text { New Jersey } \\
\text { New York (Upstate) } \\
\text { New York City } \\
\text { Pennsylvania }\end{array}$ & $\begin{array}{r}36 \\
27 \\
3 \\
6\end{array}$ & $\begin{array}{r}62 \\
9 \\
24 \\
15 \\
15\end{array}$ & $\begin{array}{r}254 \\
13 \\
227 \\
29 \\
31\end{array}$ & $\begin{array}{r}2,837 \\
339 \\
1,058 \\
755 \\
685\end{array}$ & $\begin{array}{r}3,031 \\
404 \\
1,061 \\
788 \\
778\end{array}$ & $\begin{array}{r}392 \\
56 \\
98 \\
134 \\
104\end{array}$ & $\begin{array}{l}647 \\
103 \\
122 \\
173 \\
221\end{array}$ & $\begin{array}{r}1,014 \\
164 \\
455 \\
382 \\
399\end{array}$ & $\begin{array}{r}28,082 \\
4,428 \\
5,408 \\
8,346 \\
9,900\end{array}$ & $\begin{array}{r}29,446 \\
4,932 \\
5,949 \\
8,960 \\
9,605\end{array}$ & $\frac{1}{-}$ & $\begin{array}{l}7 \\
0 \\
3 \\
1 \\
3\end{array}$ & $\begin{array}{r}30 \\
4 \\
27 \\
6 \\
8\end{array}$ & $\begin{array}{r}321 \\
122 \\
71 \\
128\end{array}$ & $\begin{array}{r}375 \\
78 \\
104 \\
71 \\
122\end{array}$ \\
\hline $\begin{array}{l}\text { E.N. Central } \\
\text { Illinois } \\
\text { Indiana } \\
\text { Michigan } \\
\text { Ohio } \\
\text { Wisconsin }\end{array}$ & $\begin{array}{r}23 \\
N \\
3 \\
20 \\
-\end{array}$ & $\begin{array}{r}47 \\
9 \\
0 \\
13 \\
16 \\
10\end{array}$ & $\begin{array}{r}81 \\
21 \\
0 \\
37 \\
32 \\
40\end{array}$ & $\begin{array}{r}2,145 \\
358 \\
N \\
593 \\
726 \\
468\end{array}$ & $\begin{array}{r}2,952 \\
691 \\
\mathrm{~N} \\
703 \\
701 \\
857\end{array}$ & $\begin{array}{r}465 \\
129 \\
113 \\
150 \\
3 \\
70\end{array}$ & $\begin{array}{r}1,266 \\
377 \\
161 \\
261 \\
305 \\
135\end{array}$ & $\begin{array}{r}7,047 \\
710 \\
244 \\
5,880 \\
648 \\
172\end{array}$ & $\begin{array}{r}55,459 \\
17,368 \\
7,483 \\
12,615 \\
12,192 \\
5,801\end{array}$ & $\begin{array}{r}57,246 \\
17,336 \\
7,014 \\
9,687 \\
18,144 \\
5,065\end{array}$ & $\begin{array}{l}\frac{1}{-} \\
\frac{1}{-}\end{array}$ & $\begin{array}{l}5 \\
1 \\
1 \\
0 \\
2 \\
0\end{array}$ & $\begin{array}{r}14 \\
6 \\
11 \\
3 \\
6 \\
4\end{array}$ & $\begin{array}{r}239 \\
47 \\
72 \\
19 \\
74 \\
27\end{array}$ & $\begin{array}{r}328 \\
111 \\
56 \\
22 \\
99 \\
40\end{array}$ \\
\hline $\begin{array}{l}\text { W.N. Central } \\
\text { lowa } \\
\text { Kansas } \\
\text { Minnesota } \\
\text { Missouri } \\
\text { Nebraska }{ }^{\dagger} \\
\text { North Dakota } \\
\text { South Dakota }\end{array}$ & $\begin{array}{l}8 \\
1 \\
- \\
-6 \\
1 \\
-\end{array}$ & $\begin{array}{r}28 \\
5 \\
3 \\
1 \\
9 \\
2 \\
0 \\
1\end{array}$ & $\begin{array}{r}260 \\
15 \\
11 \\
238 \\
28 \\
9 \\
7 \\
5\end{array}$ & $\begin{array}{r}1,564 \\
249 \\
172 \\
481 \\
480 \\
102 \\
17 \\
63\end{array}$ & $\begin{array}{r}1,982 \\
248 \\
187 \\
859 \\
454 \\
111 \\
14 \\
109\end{array}$ & $\begin{array}{r}\frac{65}{31} \\
- \\
- \\
33 \\
1 \\
-\end{array}$ & $\begin{array}{r}368 \\
35 \\
42 \\
62 \\
189 \\
25 \\
3 \\
6\end{array}$ & $\begin{array}{r}441 \\
62 \\
124 \\
105 \\
251 \\
56 \\
7 \\
15\end{array}$ & $\begin{array}{r}15,991 \\
1,545 \\
1,734 \\
2,510 \\
8,547 \\
1,225 \\
107 \\
323\end{array}$ & $\begin{array}{r}16,248 \\
1,406 \\
2,250 \\
3,024 \\
8,162 \\
1,006 \\
97 \\
303\end{array}$ & $\begin{array}{l}- \\
- \\
- \\
- \\
-\end{array}$ & $\begin{array}{l}2 \\
0 \\
0 \\
0 \\
0 \\
0 \\
0 \\
0\end{array}$ & $\begin{array}{r}15 \\
1 \\
3 \\
9 \\
6 \\
2 \\
3 \\
0\end{array}$ & $\begin{array}{r}133 \\
1 \\
14 \\
71 \\
32 \\
8 \\
7 \\
-\end{array}$ & $\begin{array}{r}99 \\
13 \\
40 \\
30 \\
14 \\
2 \\
-\end{array}$ \\
\hline $\begin{array}{l}\text { S. Atlantic } \\
\text { Delaware } \\
\text { District of Columbia } \\
\text { Florida } \\
\text { Georgia } \\
\text { Maryland }^{\dagger} \\
\text { North Carolina } \text { South Carolina }^{\dagger} \\
\text { Virginia }^{\dagger} \\
\text { West Virginia }^{\text {West }}\end{array}$ & $\begin{array}{l}38 \\
- \\
36 \\
-2 \\
\text { N } \\
- \\
-\end{array}$ & $\begin{array}{r}50 \\
1 \\
1 \\
19 \\
11 \\
3 \\
0 \\
1 \\
9 \\
0\end{array}$ & $\begin{array}{r}95 \\
4 \\
4 \\
44 \\
26 \\
11 \\
0 \\
7 \\
50 \\
6\end{array}$ & $\begin{array}{r}2,284 \\
35 \\
55 \\
986 \\
492 \\
186 \\
\mathrm{~N} \\
89 \\
415 \\
26\end{array}$ & $\begin{array}{r}2,407 \\
50 \\
49 \\
854 \\
643 \\
190 \\
\mathrm{~N} \\
98 \\
482 \\
41\end{array}$ & $\begin{array}{r}941 \\
24 \\
17 \\
316 \\
4 \\
50 \\
348 \\
110 \\
69 \\
3\end{array}$ & $\begin{array}{r}1,592 \\
27 \\
35 \\
450 \\
313 \\
126 \\
310 \\
141 \\
132 \\
17\end{array}$ & $\begin{array}{r}2,334 \\
44 \\
61 \\
548 \\
1,014 \\
186 \\
766 \\
704 \\
288 \\
42\end{array}$ & $\begin{array}{r}71,548 \\
1,287 \\
1,460 \\
19,960 \\
13,795 \\
5,564 \\
15,286 \\
7,607 \\
5,764 \\
825\end{array}$ & $\begin{array}{r}67,257 \\
771 \\
1,835 \\
17,242 \\
12,833 \\
6,066 \\
13,358 \\
7,287 \\
7,248 \\
617\end{array}$ & $\begin{array}{l}\frac{5}{-} \\
\frac{4}{1} \\
- \\
- \\
-\end{array}$ & $\begin{array}{r}10 \\
0 \\
0 \\
3 \\
2 \\
1 \\
0 \\
0 \\
1 \\
0\end{array}$ & $\begin{array}{r}24 \\
1 \\
2 \\
9 \\
6 \\
5 \\
9 \\
3 \\
8 \\
4\end{array}$ & $\begin{array}{r}459 \\
1 \\
7 \\
150 \\
87 \\
60 \\
49 \\
29 \\
57 \\
19\end{array}$ & $\begin{array}{r}455 \\
8 \\
113 \\
96 \\
64 \\
71 \\
32 \\
46 \\
25\end{array}$ \\
\hline $\begin{array}{l}\text { E.S. Central } \\
\text { Alabama }^{\dagger} \\
\text { Kentucky } \\
\text { Mississippi } \\
\text { Tennessee }^{\dagger}\end{array}$ & $\begin{array}{r}18 \\
17 \\
\mathrm{~N} \\
1\end{array}$ & $\begin{array}{l}8 \\
5 \\
0 \\
0 \\
4\end{array}$ & $\begin{array}{r}41 \\
29 \\
0 \\
0 \\
12\end{array}$ & $\begin{array}{r}449 \\
251 \\
N \\
198\end{array}$ & $\begin{array}{r}369 \\
172 \\
\mathrm{~N} \\
197\end{array}$ & $\begin{array}{r}497 \\
10 \\
168 \\
137 \\
182\end{array}$ & $\begin{array}{r}558 \\
185 \\
55 \\
143 \\
193\end{array}$ & $\begin{array}{l}866 \\
311 \\
132 \\
436 \\
237\end{array}$ & $\begin{array}{r}25,867 \\
8,248 \\
2,648 \\
6,477 \\
8,494\end{array}$ & $\begin{array}{r}24,234 \\
7,929 \\
2,674 \\
6,148 \\
7,483\end{array}$ & $\begin{array}{l}- \\
- \\
-\end{array}$ & $\begin{array}{l}2 \\
0 \\
0 \\
0 \\
1\end{array}$ & $\begin{array}{l}7 \\
5 \\
1 \\
1 \\
4\end{array}$ & $\begin{array}{r}89 \\
21 \\
4 \\
3 \\
61\end{array}$ & $\begin{array}{r}106 \\
17 \\
12 \\
77\end{array}$ \\
\hline $\begin{array}{l}\text { W.S. Central } \\
\text { Arkansas } \\
\text { Louisiana } \\
\text { Oklahoma } \\
\text { Texas }^{\dagger}\end{array}$ & $\begin{array}{r}8 \\
5 \\
-3 \\
\mathrm{~N}\end{array}$ & $\begin{array}{l}6 \\
2 \\
0 \\
2 \\
0\end{array}$ & $\begin{array}{r}31 \\
8 \\
5 \\
24 \\
0\end{array}$ & $\begin{array}{r}267 \\
121 \\
29 \\
117 \\
\mathrm{~N}\end{array}$ & $\begin{array}{r}294 \\
76 \\
57 \\
161 \\
\mathrm{~N}\end{array}$ & $\begin{array}{r}469 \\
71 \\
46 \\
124 \\
228\end{array}$ & $\begin{array}{r}913 \\
81 \\
158 \\
79 \\
567\end{array}$ & $\begin{array}{r}1,430 \\
142 \\
354 \\
764 \\
915\end{array}$ & $\begin{array}{r}41,253 \\
3,715 \\
7,241 \\
4,044 \\
26,253\end{array}$ & $\begin{array}{r}39,236 \\
3,918 \\
8,293 \\
4,068 \\
22,957\end{array}$ & $\begin{array}{l}- \\
- \\
-\end{array}$ & $\begin{array}{l}1 \\
0 \\
0 \\
1 \\
0\end{array}$ & $\begin{array}{r}15 \\
2 \\
3 \\
14 \\
1\end{array}$ & $\begin{array}{r}57 \\
7 \\
10 \\
40 \\
-\end{array}$ & $\begin{array}{r}102 \\
7 \\
33 \\
55 \\
7\end{array}$ \\
\hline $\begin{array}{l}\text { Mountain } \\
\text { Arizona } \\
\text { Colorado } \\
\text { Idaho }^{\dagger} \\
\text { Montana }^{\dagger} \\
\text { Nevada }^{\dagger} \\
\text { New Mexico } \\
\text { Utah } \\
\text { Wyoming }\end{array}$ & $\begin{array}{l}12 \\
7 \\
2 \\
- \\
- \\
- \\
3\end{array}$ & $\begin{array}{r}30 \\
3 \\
9 \\
3 \\
2 \\
2 \\
1 \\
7 \\
1\end{array}$ & $\begin{array}{r}66 \\
36 \\
33 \\
12 \\
11 \\
8 \\
6 \\
19 \\
4\end{array}$ & $\begin{array}{r}1,436 \\
137 \\
479 \\
159 \\
94 \\
85 \\
57 \\
390 \\
35\end{array}$ & $\begin{array}{r}1,352 \\
131 \\
470 \\
137 \\
65 \\
103 \\
81 \\
341 \\
24\end{array}$ & $\begin{array}{r}164 \\
73 \\
40 \\
- \\
- \\
- \\
13 \\
\end{array}$ & $\begin{array}{r}220 \\
92 \\
42 \\
2 \\
3 \\
25 \\
31 \\
17 \\
2\end{array}$ & $\begin{array}{r}552 \\
201 \\
90 \\
15 \\
20 \\
194 \\
65 \\
25 \\
6\end{array}$ & $\begin{array}{r}10,074 \\
4,102 \\
1,933 \\
139 \\
168 \\
1,415 \\
1,477 \\
738 \\
102\end{array}$ & $\begin{array}{r}11,579 \\
4,187 \\
2,753 \\
96 \\
133 \\
2,404 \\
1,322 \\
613 \\
71\end{array}$ & $\begin{array}{l}1 \\
- \\
-1 \\
- \\
- \\
-\end{array}$ & $\begin{array}{l}4 \\
1 \\
1 \\
0 \\
0 \\
0 \\
0 \\
0 \\
0\end{array}$ & $\begin{array}{l}8 \\
7 \\
4 \\
1 \\
0 \\
1 \\
4 \\
4 \\
1\end{array}$ & $\begin{array}{r}167 \\
77 \\
43 \\
5 \\
-1 \\
1 \\
22 \\
16 \\
3\end{array}$ & $\begin{array}{r}196 \\
97 \\
39 \\
5 \\
14 \\
24 \\
9 \\
8\end{array}$ \\
\hline $\begin{array}{l}\text { Pacific } \\
\text { Alaska } \\
\text { California } \\
\text { Hawaii } \\
\text { Oregon }^{\dagger} \\
\text { Washington }\end{array}$ & $\begin{array}{r}36 \\
2 \\
34 \\
- \\
- \\
-\end{array}$ & $\begin{array}{r}57 \\
1 \\
41 \\
1 \\
7 \\
6\end{array}$ & $\begin{array}{r}202 \\
17 \\
105 \\
3 \\
14 \\
90\end{array}$ & $\begin{array}{r}2,611 \\
95 \\
1,849 \\
40 \\
322 \\
305\end{array}$ & $\begin{array}{r}2,891 \\
99 \\
2,058 \\
57 \\
371 \\
306\end{array}$ & $\begin{array}{r}411 \\
11 \\
264 \\
1 \\
20 \\
115\end{array}$ & $\begin{array}{r}796 \\
11 \\
656 \\
18 \\
28 \\
74\end{array}$ & $\begin{array}{r}963 \\
24 \\
830 \\
29 \\
49 \\
142\end{array}$ & $\begin{array}{r}34,485 \\
493 \\
28,371 \\
773 \\
1,164 \\
3,684\end{array}$ & $\begin{array}{r}35,492 \\
508 \\
29,547 \\
893 \\
1,333 \\
3,211\end{array}$ & $\begin{array}{l}\frac{4}{4} \\
- \\
-\end{array}$ & $\begin{array}{l}2 \\
0 \\
0 \\
0 \\
1 \\
0\end{array}$ & $\begin{array}{r}15 \\
2 \\
9 \\
1 \\
6 \\
4\end{array}$ & $\begin{array}{r}100 \\
9 \\
27 \\
15 \\
47 \\
2\end{array}$ & $\begin{array}{r}130 \\
27 \\
52 \\
9 \\
42 \\
-\end{array}$ \\
\hline $\begin{array}{l}\text { American Samoa } \\
\text { C.N.M.I. } \\
\text { Guam } \\
\text { Puerto Rico } \\
\text { U.S. Virgin Islands }\end{array}$ & $\begin{array}{l}U \\
U \\
- \\
-\end{array}$ & $\begin{array}{l}0 \\
0 \\
0 \\
1 \\
0\end{array}$ & $\begin{array}{r}0 \\
0 \\
0 \\
12 \\
0\end{array}$ & $\frac{U}{\frac{U}{68}}$ & $\begin{array}{r}U \\
U \\
11 \\
238 \\
-\end{array}$ & $\frac{U}{U} \frac{U}{10}$ & $\begin{array}{l}0 \\
0 \\
1 \\
5 \\
0\end{array}$ & $\begin{array}{r}2 \\
0 \\
15 \\
16 \\
5\end{array}$ & $\begin{array}{r}U \\
\begin{array}{r}U \\
239 \\
30\end{array}\end{array}$ & $\begin{array}{r}U \\
U \\
80 \\
316 \\
45\end{array}$ & $\begin{array}{l}U \\
U \\
- \\
-\end{array}$ & $\begin{array}{l}0 \\
0 \\
0 \\
0 \\
0\end{array}$ & $\begin{array}{l}0 \\
0 \\
1 \\
0 \\
0\end{array}$ & $\begin{array}{l}U \\
U \\
- \\
-\end{array}$ & $\begin{array}{r}U \\
U \\
13 \\
4 \\
-\end{array}$ \\
\hline
\end{tabular}

C.N.M.I.: Commonwealth of Northern Mariana Islands.

U: Unavailable. ․: No reported cases. N: Not notifiable. Cum: Cumulative year-to-date counts. Med: Median. Max: Maximum.

* Incidence data for reporting year 2006 is provisional.

${ }^{\dagger}$ Contains data reported through the National Electronic Disease Surveillance System (NEDSS). 
TABLE II. (Continued) Provisional cases of selected notifiable diseases, United States, weeks ending November 11, 2006, and November 12, 2005 (45th Week)*

\begin{tabular}{|c|c|c|c|c|c|c|c|c|c|c|c|c|c|c|c|}
\hline \multirow[b]{4}{*}{ Reporting area } & \multicolumn{10}{|c|}{ Hepatitis (viral, acute), by type } & \multirow{2}{*}{\multicolumn{5}{|c|}{ Legionellosis }} \\
\hline & \multicolumn{5}{|c|}{$\mathbf{A}$} & \multicolumn{5}{|c|}{ B } & & & & & \\
\hline & \multirow{2}{*}{$\begin{array}{c}\text { Current } \\
\text { week }\end{array}$} & \multicolumn{2}{|c|}{$\begin{array}{l}\text { Previous } \\
52 \text { weeks }\end{array}$} & \multirow{2}{*}{$\begin{array}{l}\text { Cum } \\
2006 \\
\end{array}$} & \multirow{2}{*}{$\begin{array}{l}\text { Cum } \\
2005 \\
\end{array}$} & \multirow{2}{*}{$\begin{array}{c}\text { Current } \\
\text { week }\end{array}$} & \multicolumn{2}{|c|}{$\begin{array}{l}\text { Previous } \\
52 \text { weeks }\end{array}$} & \multirow{2}{*}{$\begin{array}{l}\text { Cum } \\
2006 \\
\end{array}$} & \multirow{2}{*}{$\begin{array}{l}\text { Cum } \\
2005 \\
\end{array}$} & \multirow{2}{*}{$\begin{array}{c}\text { Current } \\
\text { week }\end{array}$} & \multicolumn{2}{|c|}{$\begin{array}{l}\text { Previous } \\
52 \text { weeks }\end{array}$} & \multirow{2}{*}{$\begin{array}{c}\text { Cum } \\
2006 \\
\end{array}$} & \multirow{2}{*}{$\begin{array}{l}\text { Cum } \\
2005 \\
\end{array}$} \\
\hline & & Med & $\operatorname{Max}$ & & & & Med & $\operatorname{Max}$ & & & & Med & Max & & \\
\hline United States & 12 & 62 & 245 & 2,788 & 3,684 & 24 & 84 & 574 & 3,486 & 4,207 & 31 & 43 & 127 & 2,073 & 1,917 \\
\hline $\begin{array}{l}\text { New England } \\
\text { Connecticut } \\
\text { Maine }^{\dagger} \\
\text { Massachusetts } \\
\text { New Hampshire } \\
\text { Rhode Island } \\
\text { Vermont }^{\dagger}\end{array}$ & $\begin{array}{l}- \\
- \\
- \\
-\end{array}$ & $\begin{array}{l}3 \\
1 \\
0 \\
1 \\
0 \\
0 \\
0\end{array}$ & $\begin{array}{r}20 \\
2 \\
2 \\
6 \\
16 \\
4 \\
2\end{array}$ & $\begin{array}{r}152 \\
37 \\
6 \\
51 \\
37 \\
12 \\
9\end{array}$ & $\begin{array}{r}426 \\
47 \\
4 \\
274 \\
80 \\
15 \\
6\end{array}$ & $\begin{array}{l}- \\
- \\
- \\
-\end{array}$ & $\begin{array}{l}2 \\
1 \\
0 \\
0 \\
0 \\
0 \\
0\end{array}$ & $\begin{array}{l}8 \\
3 \\
2 \\
5 \\
2 \\
4 \\
1\end{array}$ & $\begin{array}{r}85 \\
29 \\
18 \\
14 \\
13 \\
9 \\
2\end{array}$ & $\begin{array}{r}135 \\
42 \\
12 \\
46 \\
27 \\
3 \\
5\end{array}$ & $\begin{array}{l}1 \\
1 \\
- \\
- \\
- \\
-\end{array}$ & $\begin{array}{l}2 \\
0 \\
0 \\
0 \\
0 \\
0 \\
0\end{array}$ & $\begin{array}{r}12 \\
9 \\
2 \\
4 \\
1 \\
10 \\
2\end{array}$ & $\begin{array}{r}110 \\
46 \\
8 \\
27 \\
1 \\
21 \\
7\end{array}$ & $\begin{array}{r}142 \\
33 \\
7 \\
63 \\
9 \\
21 \\
9\end{array}$ \\
\hline $\begin{array}{l}\text { Mid. Atlantic } \\
\text { New Jersey } \\
\text { New York (Upstate) } \\
\text { New York City } \\
\text { Pennsylvania }\end{array}$ & $\begin{array}{l}- \\
- \\
-\end{array}$ & $\begin{array}{l}7 \\
2 \\
1 \\
2 \\
1\end{array}$ & $\begin{array}{r}17 \\
6 \\
14 \\
10 \\
5\end{array}$ & $\begin{array}{r}316 \\
71 \\
81 \\
107 \\
57\end{array}$ & $\begin{array}{r}582 \\
132 \\
86 \\
273 \\
91\end{array}$ & $\begin{array}{l}- \\
- \\
-\end{array}$ & $\begin{array}{l}8 \\
2 \\
1 \\
2 \\
3\end{array}$ & $\begin{array}{r}55 \\
8 \\
43 \\
5 \\
9\end{array}$ & $\begin{array}{r}354 \\
85 \\
53 \\
75 \\
141\end{array}$ & $\begin{array}{r}577 \\
211 \\
52 \\
118 \\
196\end{array}$ & $\frac{\frac{14}{10}}{4}$ & $\begin{array}{r}14 \\
2 \\
6 \\
2 \\
5\end{array}$ & $\begin{array}{l}47 \\
10 \\
30 \\
12 \\
18\end{array}$ & $\begin{array}{r}787 \\
95 \\
297 \\
114 \\
281\end{array}$ & $\begin{array}{l}664 \\
111 \\
164 \\
109 \\
280\end{array}$ \\
\hline $\begin{array}{l}\text { E.N. Central } \\
\text { Illinois } \\
\text { Indiana } \\
\text { Michigan } \\
\text { Ohio } \\
\text { Wisconsin }\end{array}$ & $\begin{array}{l}\frac{2}{1} \\
\frac{1}{-}\end{array}$ & $\begin{array}{l}6 \\
1 \\
0 \\
2 \\
0 \\
1\end{array}$ & $\begin{array}{r}13 \\
4 \\
5 \\
8 \\
4 \\
4\end{array}$ & $\begin{array}{r}269 \\
61 \\
29 \\
94 \\
48 \\
37\end{array}$ & $\begin{array}{r}331 \\
118 \\
19 \\
102 \\
47 \\
45\end{array}$ & $\begin{array}{l}\frac{1}{-} \\
-1 \\
-\end{array}$ & $\begin{array}{l}8 \\
1 \\
0 \\
3 \\
2 \\
0\end{array}$ & $\begin{array}{r}24 \\
7 \\
17 \\
6 \\
10 \\
2\end{array}$ & $\begin{array}{r}346 \\
60 \\
47 \\
120 \\
111 \\
8\end{array}$ & $\begin{array}{r}500 \\
143 \\
33 \\
164 \\
116 \\
44\end{array}$ & $\begin{array}{l}10 \\
3 \\
1 \\
6 \\
-\end{array}$ & $\begin{array}{l}8 \\
0 \\
0 \\
2 \\
3 \\
0\end{array}$ & $\begin{array}{r}25 \\
4 \\
3 \\
9 \\
19 \\
5\end{array}$ & $\begin{array}{r}409 \\
21 \\
31 \\
119 \\
203 \\
35\end{array}$ & $\begin{array}{r}392 \\
53 \\
27 \\
107 \\
173 \\
32\end{array}$ \\
\hline $\begin{array}{l}\text { W.N. Central } \\
\text { lowa } \\
\text { Kansas } \\
\text { Minnesota } \\
\text { Missouri } \\
\text { Nebraska }^{\dagger} \\
\text { North Dakota } \\
\text { South Dakota }\end{array}$ & $\begin{array}{l}1 \\
- \\
- \\
- \\
-\end{array}$ & $\begin{array}{l}2 \\
0 \\
0 \\
0 \\
1 \\
0 \\
0 \\
0\end{array}$ & $\begin{array}{r}30 \\
2 \\
5 \\
29 \\
3 \\
3 \\
2 \\
3\end{array}$ & $\begin{array}{r}117 \\
8 \\
26 \\
16 \\
42 \\
17 \\
\frac{1}{8}\end{array}$ & $\begin{array}{r}82 \\
19 \\
16 \\
3 \\
30 \\
14 \\
-\end{array}$ & $\begin{array}{l}1 \\
- \\
- \\
-1 \\
-\end{array}$ & $\begin{array}{l}4 \\
0 \\
0 \\
0 \\
2 \\
0 \\
0 \\
0\end{array}$ & $\begin{array}{r}22 \\
3 \\
2 \\
13 \\
7 \\
2 \\
0 \\
1\end{array}$ & $\begin{array}{r}145 \\
15 \\
10 \\
23 \\
77 \\
19 \\
-1\end{array}$ & $\begin{array}{r}241 \\
25 \\
27 \\
29 \\
129 \\
24 \\
\frac{7}{7}\end{array}$ & $\begin{array}{l}1 \\
- \\
-1 \\
- \\
- \\
-\end{array}$ & $\begin{array}{l}1 \\
0 \\
0 \\
0 \\
0 \\
0 \\
0 \\
0\end{array}$ & $\begin{array}{r}15 \\
3 \\
2 \\
11 \\
3 \\
2 \\
1 \\
1\end{array}$ & $\begin{array}{r}70 \\
10 \\
5 \\
24 \\
19 \\
8 \\
4\end{array}$ & $\begin{array}{r}90 \\
7 \\
3 \\
26 \\
27 \\
4 \\
2 \\
21\end{array}$ \\
\hline $\begin{array}{l}\text { S. Atlantic } \\
\text { Delaware } \\
\text { District of Columbia } \\
\text { Florida } \\
\text { Georgia } \\
\text { Maryland }^{\dagger} \\
\text { North Carolina } \\
\text { South Carolina }^{\dagger} \\
\text { Virginia }^{\dagger} \\
\text { West Virginia }\end{array}$ & $\begin{array}{l}2 \\
- \\
- \\
- \\
- \\
-\end{array}$ & $\begin{array}{r}11 \\
0 \\
0 \\
4 \\
1 \\
1 \\
0 \\
0 \\
1 \\
0\end{array}$ & $\begin{array}{r}29 \\
2 \\
2 \\
13 \\
6 \\
6 \\
20 \\
3 \\
11 \\
3\end{array}$ & $\begin{array}{r}487 \\
10 \\
7 \\
189 \\
55 \\
59 \\
84 \\
23 \\
54 \\
6\end{array}$ & $\begin{array}{r}644 \\
6 \\
4 \\
256 \\
114 \\
66 \\
81 \\
37 \\
76 \\
4\end{array}$ & $\begin{array}{l}8 \\
- \\
6 \\
1 \\
-1 \\
- \\
-\end{array}$ & $\begin{array}{r}24 \\
1 \\
0 \\
8 \\
3 \\
3 \\
0 \\
2 \\
1 \\
0\end{array}$ & $\begin{array}{r}66 \\
4 \\
2 \\
19 \\
19 \\
9 \\
10 \\
23 \\
7 \\
18 \\
18\end{array}$ & $\begin{array}{r}1,009 \\
41 \\
7 \\
364 \\
142 \\
138 \\
143 \\
72 \\
53 \\
49\end{array}$ & $\begin{array}{r}1,214 \\
28 \\
11 \\
417 \\
181 \\
135 \\
150 \\
135 \\
122 \\
35\end{array}$ & $\begin{array}{l}5 \\
- \\
2 \\
-1 \\
2 \\
- \\
-\end{array}$ & $\begin{array}{l}8 \\
0 \\
0 \\
3 \\
0 \\
1 \\
0 \\
0 \\
1 \\
0\end{array}$ & $\begin{array}{r}19 \\
2 \\
5 \\
9 \\
4 \\
7 \\
5 \\
1 \\
7 \\
3\end{array}$ & $\begin{array}{r}379 \\
10 \\
27 \\
143 \\
18 \\
77 \\
33 \\
4 \\
54 \\
13\end{array}$ & $\begin{array}{r}358 \\
16 \\
11 \\
101 \\
33 \\
99 \\
27 \\
14 \\
39 \\
18\end{array}$ \\
\hline $\begin{array}{l}\text { E.S. Central } \\
\text { Alabama }^{\dagger} \\
\text { Kentucky } \\
\text { Mississippi } \\
\text { Tennessee }^{\dagger}\end{array}$ & $\begin{array}{r}1 \\
1 \\
- \\
-\end{array}$ & $\begin{array}{l}2 \\
0 \\
0 \\
0 \\
1\end{array}$ & $\begin{array}{l}8 \\
3 \\
5 \\
1 \\
5\end{array}$ & $\begin{array}{r}114 \\
17 \\
31 \\
7 \\
59\end{array}$ & $\begin{array}{r}227 \\
42 \\
24 \\
18 \\
143\end{array}$ & $\begin{array}{r}10 \\
9 \\
- \\
-1\end{array}$ & $\begin{array}{l}6 \\
2 \\
1 \\
0 \\
2\end{array}$ & $\begin{array}{r}16 \\
8 \\
5 \\
2 \\
7\end{array}$ & $\begin{array}{r}292 \\
100 \\
61 \\
13 \\
118\end{array}$ & $\begin{array}{r}324 \\
80 \\
62 \\
46 \\
136\end{array}$ & $\begin{array}{l}- \\
- \\
-\end{array}$ & $\begin{array}{l}1 \\
0 \\
0 \\
0 \\
1\end{array}$ & $\begin{array}{l}9 \\
2 \\
4 \\
1 \\
7\end{array}$ & $\begin{array}{r}83 \\
10 \\
32 \\
1 \\
40\end{array}$ & $\begin{array}{r}76 \\
13 \\
26 \\
3 \\
34\end{array}$ \\
\hline $\begin{array}{l}\text { W.S. Central } \\
\text { Arkansas } \\
\text { Louisiana } \\
\text { Oklahoma } \\
\text { Texas }^{\dagger}\end{array}$ & $\begin{array}{l}- \\
- \\
-\end{array}$ & $\begin{array}{l}3 \\
0 \\
0 \\
0 \\
1\end{array}$ & $\begin{array}{r}77 \\
9 \\
4 \\
2 \\
73\end{array}$ & $\begin{array}{r}150 \\
37 \\
19 \\
6 \\
88\end{array}$ & $\begin{array}{r}418 \\
18 \\
59 \\
4 \\
337\end{array}$ & $\frac{2}{-}$ & $\begin{array}{r}14 \\
1 \\
0 \\
0 \\
11\end{array}$ & $\begin{array}{r}315 \\
3 \\
5 \\
17 \\
295\end{array}$ & $\begin{array}{r}623 \\
41 \\
31 \\
60 \\
491\end{array}$ & $\begin{array}{r}548 \\
62 \\
64 \\
39 \\
383\end{array}$ & $\begin{array}{l}- \\
- \\
-\end{array}$ & $\begin{array}{l}0 \\
0 \\
0 \\
0 \\
0\end{array}$ & $\begin{array}{r}32 \\
3 \\
2 \\
3 \\
26\end{array}$ & $\begin{array}{r}43 \\
3 \\
4 \\
1 \\
35\end{array}$ & $\begin{array}{r}42 \\
6 \\
2 \\
7 \\
7 \\
27\end{array}$ \\
\hline $\begin{array}{l}\text { Mountain } \\
\text { Arizona } \\
\text { Colorado } \\
\text { Idaho }^{\dagger} \\
\text { Montana }^{\dagger} \\
\text { Nevada }^{\dagger} \\
\text { New Mexico }^{\dagger} \\
\text { Utah } \\
\text { Wyoming }\end{array}$ & $\begin{array}{l}1 \\
1 \\
- \\
- \\
- \\
- \\
-\end{array}$ & $\begin{array}{l}5 \\
2 \\
1 \\
0 \\
0 \\
0 \\
0 \\
0 \\
0\end{array}$ & $\begin{array}{r}17 \\
16 \\
4 \\
2 \\
3 \\
2 \\
3 \\
2 \\
1\end{array}$ & $\begin{array}{r}232 \\
142 \\
33 \\
9 \\
9 \\
11 \\
12 \\
13 \\
3\end{array}$ & $\begin{array}{r}292 \\
162 \\
37 \\
21 \\
8 \\
20 \\
24 \\
19 \\
1\end{array}$ & $\begin{array}{l}1 \\
-1 \\
- \\
- \\
- \\
-\end{array}$ & $\begin{array}{l}3 \\
0 \\
1 \\
0 \\
0 \\
1 \\
0 \\
0 \\
0\end{array}$ & $\begin{array}{r}16 \\
3 \\
5 \\
2 \\
7 \\
5 \\
2 \\
5 \\
1\end{array}$ & $\begin{array}{r}152 \\
35 \\
31 \\
11 \\
30 \\
18 \\
27 \\
-\end{array}$ & $\begin{array}{r}170 \\
52 \\
15 \\
3 \\
46 \\
18 \\
34 \\
2\end{array}$ & $\begin{array}{l}- \\
- \\
- \\
- \\
- \\
-\end{array}$ & $\begin{array}{l}2 \\
1 \\
0 \\
0 \\
0 \\
0 \\
0 \\
0 \\
0\end{array}$ & $\begin{array}{l}8 \\
5 \\
2 \\
3 \\
1 \\
2 \\
1 \\
6 \\
0\end{array}$ & $\begin{array}{r}114 \\
38 \\
22 \\
11 \\
5 \\
8 \\
5 \\
25 \\
-\end{array}$ & $\begin{array}{r}89 \\
22 \\
19 \\
4 \\
5 \\
19 \\
3 \\
13 \\
4\end{array}$ \\
\hline $\begin{array}{l}\text { Pacific } \\
\text { Alaska } \\
\text { California } \\
\text { Hawaii } \\
\text { Oregon }^{\dagger} \\
\text { Washington }^{\text {Washing }}\end{array}$ & $\begin{array}{l}5 \\
5 \\
- \\
-\end{array}$ & $\begin{array}{r}20 \\
0 \\
15 \\
0 \\
0 \\
1\end{array}$ & $\begin{array}{r}163 \\
0 \\
162 \\
2 \\
5 \\
13\end{array}$ & $\begin{array}{r}951 \\
-58 \\
10 \\
39 \\
44\end{array}$ & $\begin{array}{r}682 \\
4 \\
571 \\
22 \\
42 \\
43\end{array}$ & $\begin{array}{l}\frac{1}{1} \\
- \\
-\end{array}$ & $\begin{array}{r}10 \\
0 \\
8 \\
0 \\
1 \\
0\end{array}$ & $\begin{array}{r}61 \\
3 \\
41 \\
1 \\
5 \\
18\end{array}$ & $\begin{array}{r}480 \\
9 \\
364 \\
6 \\
57 \\
44\end{array}$ & $\begin{array}{r}498 \\
7 \\
334 \\
7 \\
92 \\
58\end{array}$ & $\begin{array}{l}- \\
- \\
\frac{N}{-}\end{array}$ & $\begin{array}{l}2 \\
0 \\
2 \\
0 \\
0 \\
0\end{array}$ & $\begin{array}{l}9 \\
1 \\
9 \\
0 \\
0 \\
0\end{array}$ & $\begin{array}{l}\frac{78}{78} \\
\frac{N}{N}\end{array}$ & $\begin{array}{r}64 \\
1 \\
60 \\
3 \\
\mathrm{~N} \\
-\end{array}$ \\
\hline $\begin{array}{l}\text { American Samoa } \\
\text { C.N.M.I. } \\
\text { Guam } \\
\text { Puerto Rico } \\
\text { U.S. Virgin Islands }\end{array}$ & $\begin{array}{l}U \\
U \\
- \\
-\end{array}$ & $\begin{array}{l}0 \\
0 \\
0 \\
0 \\
0\end{array}$ & $\begin{array}{l}0 \\
0 \\
0 \\
5 \\
0\end{array}$ & $\frac{U}{\frac{U}{23}}$ & $\begin{array}{r}1 \\
U \\
2 \\
60 \\
\end{array}$ & $\begin{array}{l}U \\
U \\
- \\
-\end{array}$ & $\begin{array}{l}0 \\
0 \\
0 \\
0 \\
0\end{array}$ & $\begin{array}{l}0 \\
0 \\
0 \\
8 \\
0\end{array}$ & $\frac{U}{\frac{U}{25}}$ & $\begin{array}{l}\bar{U} \\
18 \\
47 \\
-\end{array}$ & $\begin{array}{l}U \\
U \\
- \\
-\end{array}$ & $\begin{array}{l}0 \\
0 \\
0 \\
0 \\
0\end{array}$ & $\begin{array}{l}0 \\
0 \\
0 \\
1 \\
0\end{array}$ & $\begin{array}{l}U \\
\frac{U}{1} \\
-\end{array}$ & $\begin{array}{l}U \\
U \\
- \\
-\end{array}$ \\
\hline
\end{tabular}

C.N.M.I.: Commonwealth of Northern Mariana Islands

U: Unavailable. ․: No reported cases. N: Not notifiable. Cum: Cumulative year-to-date counts.

* Incidence data for reporting year 2006 is provisional.

Med: Median. Max: Maximum.

${ }^{\dagger}$ Contains data reported through the National Electronic Disease Surveillance System (NEDSS). 
TABLE II. (Continued) Provisional cases of selected notifiable diseases, United States, weeks ending November 11, 2006, and November 12, 2005 (45th Week)*

\begin{tabular}{|c|c|c|c|c|c|c|c|c|c|c|}
\hline \multirow[b]{4}{*}{ Reporting area } & \multicolumn{5}{|c|}{ Lyme disease } & \multicolumn{5}{|c|}{ Malaria } \\
\hline & \multirow{3}{*}{$\begin{array}{c}\text { Current } \\
\text { week }\end{array}$} & \multirow{2}{*}{\multicolumn{2}{|c|}{$\begin{array}{l}\text { Previous } \\
52 \text { weeks }\end{array}$}} & \multirow{3}{*}{$\begin{array}{l}\text { Cum } \\
2006 \\
\end{array}$} & \multirow{3}{*}{$\begin{array}{l}\text { Cum } \\
2005 \\
\end{array}$} & \multirow{3}{*}{$\begin{array}{c}\text { Current } \\
\text { week }\end{array}$} & \multirow{2}{*}{\multicolumn{2}{|c|}{$\begin{array}{l}\text { Previous } \\
52 \text { weeks }\end{array}$}} & \multirow{3}{*}{$\begin{array}{l}\text { Cum } \\
2006 \\
\end{array}$} & \multirow{3}{*}{$\begin{array}{l}\text { Cum } \\
2005 \\
\end{array}$} \\
\hline & & & & & & & & & & \\
\hline & & Med & Max & & & & Med & Max & & \\
\hline United States & 125 & 235 & 2,153 & 15,173 & 19,585 & 12 & 25 & 125 & 1,098 & 1,229 \\
\hline $\begin{array}{l}\text { New England } \\
\text { Connecticut } \\
\text { Maine }^{\dagger} \\
\text { Massachusetts } \\
\text { New Hampshire } \\
\text { Rhode Island } \\
\text { Vermont }^{\dagger}\end{array}$ & $\begin{array}{r}81 \\
10 \\
- \\
7 \\
62 \\
2\end{array}$ & $\begin{array}{r}30 \\
13 \\
1 \\
1 \\
5 \\
0 \\
1\end{array}$ & $\begin{array}{r}780 \\
753 \\
34 \\
23 \\
90 \\
30 \\
14\end{array}$ & $\begin{array}{r}2,573 \\
1,623 \\
220 \\
33 \\
514 \\
93 \\
90\end{array}$ & $\begin{array}{r}3,551 \\
750 \\
236 \\
2,260 \\
218 \\
37 \\
50\end{array}$ & $\begin{array}{l}- \\
- \\
- \\
-\end{array}$ & $\begin{array}{l}1 \\
0 \\
0 \\
0 \\
0 \\
0 \\
0\end{array}$ & $\begin{array}{r}11 \\
3 \\
1 \\
3 \\
3 \\
8 \\
1\end{array}$ & $\begin{array}{r}45 \\
11 \\
4 \\
19 \\
9 \\
1 \\
1\end{array}$ & $\begin{array}{r}66 \\
17 \\
5 \\
36 \\
5 \\
2 \\
1\end{array}$ \\
\hline $\begin{array}{l}\text { Mid. Atlantic } \\
\text { New Jersey } \\
\text { New York (Upstate) } \\
\text { New York City } \\
\text { Pennsylvania }\end{array}$ & $\begin{array}{r}28 \\
3 \\
22 \\
-3\end{array}$ & $\begin{array}{r}137 \\
22 \\
64 \\
0 \\
37\end{array}$ & $\begin{array}{r}1,176 \\
172 \\
1,150 \\
18 \\
234\end{array}$ & $\begin{array}{r}8,618 \\
1,848 \\
3,644 \\
115 \\
3,011\end{array}$ & $\begin{array}{r}11,196 \\
3,249 \\
3,529 \\
376 \\
4,042\end{array}$ & $\frac{2}{\frac{1}{1}}$ & $\begin{array}{l}5 \\
0 \\
1 \\
2 \\
1\end{array}$ & $\begin{array}{r}13 \\
3 \\
11 \\
9 \\
4\end{array}$ & $\begin{array}{r}236 \\
28 \\
42 \\
125 \\
41\end{array}$ & $\begin{array}{r}326 \\
72 \\
47 \\
174 \\
33\end{array}$ \\
\hline $\begin{array}{l}\text { E.N. Central } \\
\text { Illinois } \\
\text { Indiana } \\
\text { Michigan } \\
\text { Ohio } \\
\text { Wisconsin }\end{array}$ & $\begin{array}{l}- \\
- \\
- \\
-\end{array}$ & $\begin{array}{r}10 \\
0 \\
0 \\
1 \\
1 \\
9\end{array}$ & $\begin{array}{r}146 \\
2 \\
3 \\
6 \\
5 \\
141\end{array}$ & $\begin{array}{r}1,346 \\
17 \\
49 \\
39 \\
1,241\end{array}$ & $\begin{array}{r}1,682 \\
124 \\
30 \\
54 \\
53 \\
1,421\end{array}$ & $\begin{array}{l}- \\
- \\
- \\
-\end{array}$ & $\begin{array}{l}2 \\
1 \\
0 \\
0 \\
0 \\
0\end{array}$ & $\begin{array}{l}7 \\
4 \\
3 \\
2 \\
3 \\
3\end{array}$ & $\begin{array}{r}107 \\
44 \\
9 \\
16 \\
27 \\
11\end{array}$ & $\begin{array}{r}132 \\
70 \\
6 \\
21 \\
24 \\
11\end{array}$ \\
\hline $\begin{array}{l}\text { W.N. Central } \\
\text { lowa } \\
\text { Kansas } \\
\text { Minnesota } \\
\text { Missouri } \\
\text { Nebraska } \\
\text { North Dakota } \\
\text { South Dakota }\end{array}$ & $\begin{array}{l}- \\
- \\
- \\
- \\
-\end{array}$ & $\begin{array}{l}6 \\
0 \\
0 \\
4 \\
0 \\
0 \\
0 \\
0\end{array}$ & $\begin{array}{r}169 \\
8 \\
2 \\
167 \\
2 \\
2 \\
3 \\
1\end{array}$ & $\begin{array}{r}715 \\
83 \\
4 \\
606 \\
10 \\
11 \\
1\end{array}$ & $\begin{array}{r}831 \\
91 \\
3 \\
718 \\
14 \\
3 \\
2\end{array}$ & $\begin{array}{l}3 \\
- \\
- \\
- \\
-\end{array}$ & $\begin{array}{l}0 \\
0 \\
0 \\
0 \\
0 \\
0 \\
0 \\
0\end{array}$ & $\begin{array}{r}32 \\
1 \\
2 \\
30 \\
1 \\
1 \\
1 \\
1\end{array}$ & $\begin{array}{r}50 \\
2 \\
7 \\
29 \\
6 \\
4 \\
1 \\
1\end{array}$ & $\begin{array}{r}45 \\
8 \\
6 \\
11 \\
17 \\
3 \\
- \\
-\end{array}$ \\
\hline $\begin{array}{l}\text { S. Atlantic } \\
\text { Delaware } \\
\text { District of Columbia } \\
\text { Florida } \\
\text { Georgia } \\
\text { Maryland }^{\dagger} \\
\text { North Carolina } \\
\text { South Carolina }^{\dagger} \\
\text { Virginia }^{\dagger} \\
\text { West Virginia }^{\text {West }}\end{array}$ & $\begin{array}{r}10 \\
1 \\
-4 \\
- \\
3 \\
- \\
-\end{array}$ & $\begin{array}{r}28 \\
8 \\
0 \\
1 \\
0 \\
13 \\
0 \\
0 \\
3 \\
0\end{array}$ & $\begin{array}{r}112 \\
28 \\
7 \\
5 \\
1 \\
69 \\
4 \\
2 \\
25 \\
44\end{array}$ & $\begin{array}{r}1,631 \\
437 \\
55 \\
42 \\
6 \\
789 \\
29 \\
18 \\
242 \\
13\end{array}$ & $\begin{array}{r}2,088 \\
610 \\
8 \\
39 \\
6 \\
1,116 \\
44 \\
19 \\
230 \\
16\end{array}$ & $\begin{array}{l}4 \\
- \\
-1 \\
-3 \\
- \\
-\end{array}$ & $\begin{array}{l}7 \\
0 \\
0 \\
1 \\
1 \\
1 \\
0 \\
0 \\
1 \\
0\end{array}$ & $\begin{array}{r}15 \\
1 \\
2 \\
6 \\
6 \\
5 \\
8 \\
2 \\
9 \\
1\end{array}$ & $\begin{array}{r}290 \\
5 \\
3 \\
56 \\
75 \\
64 \\
28 \\
9 \\
48 \\
2\end{array}$ & $\begin{array}{r}269 \\
3 \\
8 \\
47 \\
47 \\
94 \\
30 \\
8 \\
29 \\
3\end{array}$ \\
\hline $\begin{array}{l}\text { E.S. Central } \\
\text { Alabama }^{\dagger} \\
\text { Kentucky } \\
\text { Mississippi } \\
\text { Tennessee }^{\dagger}\end{array}$ & $\begin{array}{l}3 \\
3 \\
- \\
-\end{array}$ & $\begin{array}{l}0 \\
0 \\
0 \\
0 \\
0\end{array}$ & $\begin{array}{l}3 \\
1 \\
2 \\
0 \\
2\end{array}$ & $\begin{array}{r}27 \\
10 \\
7 \\
10\end{array}$ & $\begin{array}{r}33 \\
3 \\
5 \\
25\end{array}$ & $\begin{array}{l}- \\
- \\
-\end{array}$ & $\begin{array}{l}0 \\
0 \\
0 \\
0 \\
0\end{array}$ & $\begin{array}{l}3 \\
2 \\
1 \\
1 \\
2\end{array}$ & $\begin{array}{r}21 \\
9 \\
3 \\
4 \\
5\end{array}$ & $\begin{array}{r}28 \\
5 \\
10 \\
13\end{array}$ \\
\hline $\begin{array}{l}\text { W.S. Central } \\
\text { Arkansas } \\
\text { Louisiana } \\
\text { Oklahoma } \\
\text { Texas }^{\dagger}\end{array}$ & $\begin{array}{l}- \\
- \\
-\end{array}$ & $\begin{array}{l}0 \\
0 \\
0 \\
0 \\
0\end{array}$ & $\begin{array}{l}3 \\
1 \\
0 \\
0 \\
3\end{array}$ & $\frac{17}{-}$ & $\begin{array}{r}74 \\
4 \\
3 \\
67\end{array}$ & $\begin{array}{l}- \\
- \\
-\end{array}$ & $\begin{array}{l}2 \\
0 \\
0 \\
0 \\
1\end{array}$ & $\begin{array}{r}31 \\
1 \\
1 \\
2 \\
29\end{array}$ & $\begin{array}{r}78 \\
2 \\
4 \\
7 \\
65\end{array}$ & $\begin{array}{r}114 \\
6 \\
5 \\
10 \\
93\end{array}$ \\
\hline $\begin{array}{l}\text { Mountain } \\
\text { Arizona } \\
\text { Colorado } \\
\text { Idaho }^{\dagger} \\
\text { Montana }^{\dagger} \\
\text { Nevada }^{\dagger} \\
\text { New Mexico }^{\dagger} \\
\text { Utah } \\
\text { Wyoming }\end{array}$ & $\begin{array}{l}- \\
- \\
- \\
- \\
- \\
-\end{array}$ & $\begin{array}{l}0 \\
0 \\
0 \\
0 \\
0 \\
0 \\
0 \\
0 \\
0\end{array}$ & $\begin{array}{l}4 \\
2 \\
1 \\
2 \\
0 \\
1 \\
1 \\
1 \\
1\end{array}$ & $\begin{array}{r}28 \\
7 \\
5 \\
5 \\
- \\
2 \\
2 \\
6 \\
1\end{array}$ & $\begin{array}{r}21 \\
8 \\
2 \\
\\
3 \\
3 \\
2 \\
3\end{array}$ & $\begin{array}{l}1 \\
-1 \\
- \\
- \\
- \\
-\end{array}$ & $\begin{array}{l}1 \\
0 \\
0 \\
0 \\
0 \\
0 \\
0 \\
0 \\
0\end{array}$ & $\begin{array}{l}9 \\
9 \\
1 \\
1 \\
1 \\
1 \\
1 \\
2 \\
0\end{array}$ & $\begin{array}{r}63 \\
22 \\
13 \\
1 \\
2 \\
4 \\
4 \\
17 \\
-\end{array}$ & $\begin{array}{l}52 \\
13 \\
24 \\
- \\
3 \\
3 \\
7 \\
2\end{array}$ \\
\hline $\begin{array}{l}\text { Pacific } \\
\text { Alaska } \\
\text { California } \\
\text { Hawaii } \\
\text { Oregon }^{\dagger} \\
\text { Washington }^{\dagger}\end{array}$ & $\begin{array}{l}3 \\
3 \\
N \\
- \\
-\end{array}$ & $\begin{array}{l}4 \\
0 \\
4 \\
0 \\
0 \\
0\end{array}$ & $\begin{array}{r}16 \\
1 \\
15 \\
0 \\
2 \\
3\end{array}$ & $\begin{array}{r}218 \\
3 \\
202 \\
N \\
10 \\
3\end{array}$ & $\begin{array}{r}109 \\
4 \\
76 \\
N \\
20 \\
9\end{array}$ & $\begin{array}{l}\frac{2}{2} \\
- \\
-\end{array}$ & $\begin{array}{l}4 \\
0 \\
3 \\
0 \\
0 \\
0\end{array}$ & $\begin{array}{r}13 \\
4 \\
10 \\
2 \\
1 \\
5\end{array}$ & $\begin{array}{r}208 \\
23 \\
140 \\
4 \\
9 \\
32\end{array}$ & $\begin{array}{r}197 \\
5 \\
147 \\
17 \\
12 \\
16\end{array}$ \\
\hline $\begin{array}{l}\text { American Samoa } \\
\text { C.N.M.I. } \\
\text { Guam } \\
\text { Puerto Rico } \\
\text { U.S. Virgin Islands }\end{array}$ & $\frac{U}{\mathrm{U}}$ & $\begin{array}{l}0 \\
0 \\
0 \\
0 \\
0\end{array}$ & $\begin{array}{l}0 \\
0 \\
0 \\
0 \\
0\end{array}$ & $\begin{array}{l}U \\
\frac{U}{N} \\
\end{array}$ & $\begin{array}{l}U \\
\frac{U}{N} \\
\end{array}$ & $\begin{array}{l}U \\
U \\
- \\
-\end{array}$ & $\begin{array}{l}0 \\
0 \\
0 \\
0 \\
0\end{array}$ & $\begin{array}{l}0 \\
0 \\
0 \\
0 \\
0\end{array}$ & $\begin{array}{l}U \\
U \\
- \\
-\end{array}$ & $\frac{U}{U}$ \\
\hline
\end{tabular}

C.N.M.I: Commonwealth of Northern Mariana Islands.

* Incidence data for reporting year 2006 is provisional.

Cum: Cumulative year-to-date counts. Med: Median. Max: Maximum.

Contains data reported through the National Electronic Disease Surveillance System (NEDSS). 
TABLE II. (Continued) Provisional cases of selected notifiable diseases, United States, weeks ending November 11, 2006, and November 12, 2005 (45th Week)*

\begin{tabular}{|c|c|c|c|c|c|c|c|c|c|c|c|c|c|c|c|}
\hline \multirow[b]{4}{*}{ Reporting area } & \multicolumn{10}{|c|}{ Meningococcal disease, invasive } & \multirow{2}{*}{\multicolumn{5}{|c|}{ Pertussis }} \\
\hline & \multicolumn{5}{|c|}{ All serogroups } & \multicolumn{5}{|c|}{ Serogroup unknown } & & & & & \\
\hline & \multirow{2}{*}{$\begin{array}{c}\text { Current } \\
\text { week }\end{array}$} & \multicolumn{2}{|c|}{$\begin{array}{c}\text { Previous } \\
52 \text { weeks } \\
\end{array}$} & \multirow{2}{*}{$\begin{array}{l}\text { Cum } \\
2006 \\
\end{array}$} & \multirow{2}{*}{$\begin{array}{l}\text { Cum } \\
2005 \\
\end{array}$} & \multirow{2}{*}{$\begin{array}{c}\text { Current } \\
\text { week }\end{array}$} & \multicolumn{2}{|c|}{$\begin{array}{l}\text { Previous } \\
52 \text { weeks } \\
\end{array}$} & \multirow{2}{*}{$\begin{array}{l}\text { Cum } \\
2006 \\
\end{array}$} & \multirow{2}{*}{$\begin{array}{l}\text { Cum } \\
2005 \\
\end{array}$} & \multirow{2}{*}{$\begin{array}{c}\text { Current } \\
\text { week }\end{array}$} & \multicolumn{2}{|c|}{$\begin{array}{l}\text { Previous } \\
52 \text { weeks } \\
\end{array}$} & \multirow{2}{*}{$\begin{array}{l}\text { Cum } \\
2006 \\
\end{array}$} & \multirow{2}{*}{$\begin{array}{l}\text { Cum } \\
2005 \\
\end{array}$} \\
\hline & & Med & Max & & & & Med & Max & & & & Med & Max & & \\
\hline United States & 11 & 19 & 85 & 869 & 1,047 & 7 & 12 & 58 & 579 & 647 & 93 & 258 & 2,877 & 11,035 & 19,933 \\
\hline $\begin{array}{l}\text { New England } \\
\text { Connecticut } \\
\text { Maine }^{\dagger} \\
\text { Massachusetts } \\
\text { New Hampshire } \\
\text { Rhode Island } \\
\text { Vermont }^{\dagger}\end{array}$ & $\begin{array}{l}1 \\
1 \\
- \\
- \\
-\end{array}$ & $\begin{array}{l}1 \\
0 \\
0 \\
0 \\
0 \\
0 \\
0\end{array}$ & $\begin{array}{l}3 \\
2 \\
1 \\
2 \\
2 \\
1 \\
1\end{array}$ & $\begin{array}{r}41 \\
10 \\
6 \\
15 \\
6 \\
6 \\
2 \\
2\end{array}$ & $\begin{array}{r}64 \\
12 \\
2 \\
30 \\
12 \\
3 \\
5\end{array}$ & $\begin{array}{l}1 \\
1 \\
- \\
- \\
- \\
-\end{array}$ & $\begin{array}{l}0 \\
0 \\
0 \\
0 \\
0 \\
0 \\
0\end{array}$ & $\begin{array}{l}2 \\
2 \\
1 \\
2 \\
2 \\
0 \\
0\end{array}$ & $\begin{array}{r}28 \\
3 \\
4 \\
15 \\
6 \\
- \\
-\end{array}$ & $\begin{array}{r}22 \\
1 \\
2 \\
5 \\
12 \\
2\end{array}$ & $\begin{array}{l}\frac{3}{-} \\
\frac{1}{2} \\
2\end{array}$ & $\begin{array}{r}26 \\
1 \\
1 \\
17 \\
2 \\
0 \\
1\end{array}$ & $\begin{array}{r}83 \\
5 \\
11 \\
43 \\
36 \\
17 \\
14\end{array}$ & $\begin{array}{r}1,007 \\
37 \\
73 \\
594 \\
153 \\
49 \\
101\end{array}$ & $\begin{array}{r}1,260 \\
62 \\
47 \\
955 \\
80 \\
36 \\
80\end{array}$ \\
\hline $\begin{array}{l}\text { Mid. Atlantic } \\
\text { New Jersey } \\
\text { New York (Upstate) } \\
\text { New York City } \\
\text { Pennsylvania }\end{array}$ & $\begin{array}{l}- \\
- \\
-\end{array}$ & $\begin{array}{l}2 \\
0 \\
0 \\
1 \\
0\end{array}$ & $\begin{array}{r}13 \\
1 \\
7 \\
4 \\
5\end{array}$ & $\begin{array}{l}91 \\
- \\
53 \\
38\end{array}$ & $\begin{array}{r}133 \\
31 \\
34 \\
23 \\
45\end{array}$ & $\begin{array}{l}- \\
- \\
-\end{array}$ & $\begin{array}{l}1 \\
0 \\
0 \\
1 \\
0\end{array}$ & $\begin{array}{r}11 \\
1 \\
5 \\
4 \\
5\end{array}$ & $\begin{array}{l}87 \\
- \\
53 \\
34\end{array}$ & $\begin{array}{r}103 \\
31 \\
12 \\
23 \\
37\end{array}$ & $\frac{21}{\frac{14}{7}}$ & $\begin{array}{r}35 \\
4 \\
15 \\
1 \\
13\end{array}$ & $\begin{array}{r}137 \\
13 \\
123 \\
8 \\
26\end{array}$ & $\begin{array}{r}1,598 \\
184 \\
738 \\
64 \\
612\end{array}$ & $\begin{array}{r}1,151 \\
162 \\
443 \\
96 \\
450\end{array}$ \\
\hline $\begin{array}{l}\text { E.N. Central } \\
\text { Illinois } \\
\text { Indiana } \\
\text { Michigan } \\
\text { Ohio } \\
\text { Wisconsin }\end{array}$ & $\begin{array}{l}3 \\
1 \\
1 \\
1 \\
-\end{array}$ & $\begin{array}{l}2 \\
0 \\
0 \\
0 \\
1 \\
0\end{array}$ & $\begin{array}{r}11 \\
4 \\
5 \\
3 \\
5 \\
2\end{array}$ & $\begin{array}{r}104 \\
18 \\
21 \\
20 \\
42 \\
3\end{array}$ & $\begin{array}{r}138 \\
31 \\
18 \\
31 \\
36 \\
22\end{array}$ & $\begin{array}{l}2 \\
1 \\
1 \\
-\end{array}$ & $\begin{array}{l}1 \\
0 \\
0 \\
0 \\
1 \\
0\end{array}$ & $\begin{array}{l}6 \\
4 \\
1 \\
1 \\
4 \\
2\end{array}$ & $\begin{array}{r}72 \\
18 \\
8 \\
9 \\
34 \\
3\end{array}$ & $\begin{array}{r}111 \\
31 \\
8 \\
18 \\
32 \\
22\end{array}$ & $\begin{array}{r}24 \\
4 \\
7 \\
13 \\
-\end{array}$ & $\begin{array}{r}39 \\
6 \\
4 \\
9 \\
12 \\
4\end{array}$ & $\begin{array}{r}133 \\
23 \\
75 \\
37 \\
30 \\
21\end{array}$ & $\begin{array}{r}1,638 \\
231 \\
213 \\
506 \\
524 \\
164\end{array}$ & $\begin{array}{r}3,393 \\
816 \\
289 \\
274 \\
1,011 \\
1,003\end{array}$ \\
\hline $\begin{array}{l}\text { W.N. Central } \\
\text { lowa } \\
\text { Kansas } \\
\text { Minnesota } \\
\text { Missouri } \\
\text { Nebraska }^{\dagger} \\
\text { North Dakota } \\
\text { South Dakota }\end{array}$ & $\begin{array}{l}- \\
- \\
- \\
- \\
-\end{array}$ & $\begin{array}{l}1 \\
0 \\
0 \\
0 \\
0 \\
0 \\
0 \\
0\end{array}$ & $\begin{array}{l}4 \\
2 \\
1 \\
2 \\
2 \\
2 \\
2 \\
1 \\
1\end{array}$ & $\begin{array}{r}55 \\
17 \\
2 \\
13 \\
14 \\
6 \\
1 \\
2\end{array}$ & $\begin{array}{r}71 \\
15 \\
9 \\
13 \\
25 \\
5 \\
4\end{array}$ & $\begin{array}{l}- \\
- \\
- \\
- \\
-\end{array}$ & $\begin{array}{l}0 \\
0 \\
0 \\
0 \\
0 \\
0 \\
0 \\
0\end{array}$ & $\begin{array}{l}3 \\
1 \\
1 \\
1 \\
1 \\
1 \\
1 \\
0\end{array}$ & $\begin{array}{r}18 \\
5 \\
2 \\
4 \\
2 \\
4 \\
1 \\
-\end{array}$ & $\begin{array}{r}29 \\
1 \\
9 \\
5 \\
11 \\
3 \\
-\end{array}$ & $\begin{array}{l}\frac{9}{5} \\
-1 \\
3 \\
-\end{array}$ & $\begin{array}{r}24 \\
5 \\
6 \\
0 \\
6 \\
2 \\
0 \\
0\end{array}$ & $\begin{array}{r}552 \\
40 \\
25 \\
485 \\
42 \\
9 \\
25 \\
4\end{array}$ & $\begin{array}{r}1,049 \\
226 \\
274 \\
161 \\
258 \\
84 \\
26 \\
20\end{array}$ & $\begin{array}{r}3,352 \\
951 \\
419 \\
966 \\
448 \\
262 \\
131 \\
175\end{array}$ \\
\hline $\begin{array}{l}\text { S. Atlantic } \\
\text { Delaware } \\
\text { District of Columbia } \\
\text { Florida } \\
\text { Georgia } \\
\text { Maryland }^{\dagger} \\
\text { North Carolina }^{-} \text {South Carolina }^{\dagger} \\
\text { Virginia }^{\dagger} \\
\text { West Virginia }^{\text {West }}\end{array}$ & $\begin{array}{l}2 \\
- \\
2 \\
- \\
- \\
-\end{array}$ & $\begin{array}{l}4 \\
0 \\
0 \\
1 \\
0 \\
0 \\
0 \\
0 \\
0 \\
0\end{array}$ & $\begin{array}{r}14 \\
1 \\
1 \\
6 \\
3 \\
2 \\
11 \\
2 \\
4 \\
2\end{array}$ & $\begin{array}{r}162 \\
4 \\
1 \\
65 \\
14 \\
12 \\
24 \\
18 \\
16 \\
8\end{array}$ & $\begin{array}{r}195 \\
4 \\
5 \\
72 \\
15 \\
21 \\
29 \\
13 \\
30 \\
6\end{array}$ & $\begin{array}{l}2 \\
- \\
2 \\
- \\
- \\
- \\
-\end{array}$ & $\begin{array}{l}1 \\
0 \\
0 \\
0 \\
0 \\
0 \\
0 \\
0 \\
0 \\
0\end{array}$ & $\begin{array}{l}7 \\
1 \\
1 \\
5 \\
3 \\
1 \\
3 \\
2 \\
3 \\
0\end{array}$ & $\begin{array}{r}67 \\
4 \\
1 \\
24 \\
14 \\
2 \\
7 \\
8 \\
7 \\
-\end{array}$ & $\begin{array}{r}87 \\
4 \\
4 \\
29 \\
15 \\
4 \\
7 \\
8 \\
14 \\
2\end{array}$ & $\begin{array}{l}\frac{10}{-} \\
\frac{2}{1} \\
\frac{1}{6} \\
-1\end{array}$ & $\begin{array}{r}19 \\
0 \\
0 \\
4 \\
0 \\
3 \\
0 \\
3 \\
2 \\
0\end{array}$ & $\begin{array}{r}46 \\
1 \\
3 \\
9 \\
3 \\
9 \\
22 \\
11 \\
27 \\
9\end{array}$ & $\begin{array}{r}892 \\
3 \\
6 \\
191 \\
19 \\
114 \\
177 \\
156 \\
183 \\
43\end{array}$ & $\begin{array}{r}1,264 \\
15 \\
7 \\
185 \\
45 \\
181 \\
98 \\
374 \\
315 \\
44\end{array}$ \\
\hline $\begin{array}{l}\text { E.S. Central } \\
\text { Alabama }^{\dagger} \\
\text { Kentucky } \\
\text { Mississippi } \\
\text { Tennessee }^{\dagger}\end{array}$ & $\begin{array}{l}- \\
- \\
-\end{array}$ & $\begin{array}{l}1 \\
0 \\
0 \\
0 \\
0\end{array}$ & $\begin{array}{l}4 \\
1 \\
2 \\
1 \\
2\end{array}$ & $\begin{array}{r}36 \\
6 \\
8 \\
3 \\
19\end{array}$ & $\begin{array}{r}52 \\
5 \\
17 \\
6 \\
24\end{array}$ & $\begin{array}{l}- \\
- \\
-\end{array}$ & $\begin{array}{l}1 \\
0 \\
0 \\
0 \\
0\end{array}$ & $\begin{array}{l}4 \\
1 \\
2 \\
1 \\
2\end{array}$ & $\begin{array}{r}28 \\
4 \\
8 \\
3 \\
13\end{array}$ & $\begin{array}{r}41 \\
3 \\
17 \\
6 \\
15\end{array}$ & $\begin{array}{l}3 \\
2 \\
- \\
1\end{array}$ & $\begin{array}{l}7 \\
1 \\
1 \\
1 \\
3\end{array}$ & $\begin{array}{r}27 \\
18 \\
5 \\
4 \\
10\end{array}$ & $\begin{array}{r}326 \\
94 \\
54 \\
38 \\
140\end{array}$ & $\begin{array}{r}459 \\
75 \\
139 \\
54 \\
191\end{array}$ \\
\hline $\begin{array}{l}\text { W.S. Central } \\
\text { Arkansas } \\
\text { Louisiana } \\
\text { Oklahoma } \\
\text { Texas }^{\dagger}\end{array}$ & $\frac{3}{-}$ & $\begin{array}{l}1 \\
0 \\
0 \\
0 \\
0\end{array}$ & $\begin{array}{r}23 \\
3 \\
2 \\
4 \\
16\end{array}$ & $\begin{array}{r}55 \\
9 \\
6 \\
11 \\
29\end{array}$ & $\begin{array}{l}99 \\
14 \\
29 \\
14 \\
42\end{array}$ & $\begin{array}{l}- \\
- \\
-\end{array}$ & $\begin{array}{l}0 \\
0 \\
0 \\
0 \\
0\end{array}$ & $\begin{array}{l}6 \\
2 \\
1 \\
0 \\
4\end{array}$ & $\begin{array}{r}23 \\
6 \\
3 \\
14\end{array}$ & $\begin{array}{r}24 \\
3 \\
6 \\
2 \\
13\end{array}$ & $\begin{array}{r}4 \\
3 \\
1 \\
-\end{array}$ & $\begin{array}{r}15 \\
2 \\
0 \\
0 \\
13\end{array}$ & $\begin{array}{r}360 \\
21 \\
3 \\
124 \\
215\end{array}$ & $\begin{array}{r}619 \\
70 \\
13 \\
19 \\
517\end{array}$ & $\begin{array}{r}2,104 \\
281 \\
46 \\
1 \\
1,776\end{array}$ \\
\hline $\begin{array}{l}\text { Mountain } \\
\text { Arizona } \\
\text { Colorado } \\
\text { Idaho }^{\dagger} \\
\text { Montana }^{\dagger} \\
\text { Nevada }^{\dagger} \\
\text { New Mexico } \\
\text { Utah } \\
\text { Wyoming }\end{array}$ & $\begin{array}{l}- \\
- \\
- \\
- \\
- \\
-\end{array}$ & $\begin{array}{l}1 \\
0 \\
0 \\
0 \\
0 \\
0 \\
0 \\
0 \\
0\end{array}$ & $\begin{array}{l}5 \\
3 \\
2 \\
1 \\
1 \\
1 \\
1 \\
1 \\
2\end{array}$ & $\begin{array}{r}61 \\
17 \\
19 \\
3 \\
4 \\
3 \\
6 \\
5 \\
4\end{array}$ & $\begin{array}{r}82 \\
31 \\
17 \\
6 \\
-12 \\
5 \\
11 \\
-\end{array}$ & $\begin{array}{l}- \\
- \\
- \\
- \\
- \\
-\end{array}$ & $\begin{array}{l}0 \\
0 \\
0 \\
0 \\
0 \\
0 \\
0 \\
0 \\
0\end{array}$ & $\begin{array}{l}4 \\
3 \\
1 \\
1 \\
1 \\
0 \\
1 \\
0 \\
2\end{array}$ & $\begin{array}{r}30 \\
17 \\
2 \\
2 \\
2 \\
-3 \\
4\end{array}$ & $\begin{array}{l}23 \\
10 \\
5 \\
- \\
2 \\
4 \\
2 \\
-\end{array}$ & $\begin{array}{r}19 \\
10 \\
9 \\
- \\
- \\
- \\
- \\
-\end{array}$ & $\begin{array}{r}56 \\
8 \\
14 \\
2 \\
2 \\
0 \\
2 \\
14 \\
1\end{array}$ & $\begin{array}{r}230 \\
177 \\
40 \\
8 \\
9 \\
9 \\
6 \\
39 \\
8\end{array}$ & $\begin{array}{r}2,254 \\
436 \\
673 \\
81 \\
101 \\
54 \\
79 \\
758 \\
72\end{array}$ & $\begin{array}{r}3,591 \\
872 \\
1,180 \\
189 \\
569 \\
48 \\
169 \\
516 \\
48\end{array}$ \\
\hline $\begin{array}{l}\text { Pacific } \\
\text { Alaska } \\
\text { California } \\
\text { Hawaii } \\
\text { Oregon }^{\dagger} \\
\text { Washington }\end{array}$ & $\begin{array}{l}\frac{2}{2} \\
- \\
-\end{array}$ & $\begin{array}{l}5 \\
0 \\
3 \\
0 \\
1 \\
0\end{array}$ & $\begin{array}{r}29 \\
1 \\
14 \\
1 \\
7 \\
25\end{array}$ & $\begin{array}{r}264 \\
2 \\
165 \\
7 \\
60 \\
30\end{array}$ & $\begin{array}{r}213 \\
3 \\
135 \\
11 \\
45 \\
19\end{array}$ & $\begin{array}{l}\frac{2}{2} \\
- \\
-\end{array}$ & $\begin{array}{l}5 \\
0 \\
3 \\
0 \\
1 \\
0\end{array}$ & $\begin{array}{r}25 \\
1 \\
14 \\
1 \\
1 \\
4 \\
11\end{array}$ & $\begin{array}{r}226 \\
2 \\
165 \\
7 \\
41 \\
11\end{array}$ & $\begin{array}{r}207 \\
3 \\
135 \\
6 \\
45 \\
18\end{array}$ & $\begin{array}{l}- \\
- \\
- \\
-\end{array}$ & $\begin{array}{r}34 \\
1 \\
23 \\
1 \\
2 \\
5\end{array}$ & $\begin{array}{r}1,334 \\
15 \\
1,136 \\
4 \\
8 \\
8 \\
195\end{array}$ & $\begin{array}{r}1,652 \\
63 \\
1,151 \\
70 \\
94 \\
274\end{array}$ & $\begin{array}{r}3,359 \\
129 \\
1,667 \\
155 \\
611 \\
797\end{array}$ \\
\hline $\begin{array}{l}\text { American Samoa } \\
\text { C.N.M.I. } \\
\text { Guam } \\
\text { Puerto Rico } \\
\text { U.S. Virgin Islands }\end{array}$ & $\begin{array}{l}U \\
U \\
- \\
-\end{array}$ & $\begin{array}{l}0 \\
0 \\
0 \\
0 \\
0\end{array}$ & $\begin{array}{l}0 \\
0 \\
0 \\
0 \\
0\end{array}$ & $\begin{array}{l}- \\
- \\
-\end{array}$ & $\begin{array}{l}- \\
1 \\
7 \\
-\end{array}$ & $\begin{array}{l}U \\
U \\
- \\
-\end{array}$ & $\begin{array}{l}0 \\
0 \\
0 \\
0 \\
0\end{array}$ & $\begin{array}{l}0 \\
0 \\
0 \\
0 \\
0\end{array}$ & $\begin{array}{l}U \\
U \\
- \\
-\end{array}$ & $\begin{array}{r}U \\
U \\
1 \\
7 \\
-\end{array}$ & $\begin{array}{l}U \\
U \\
- \\
-\end{array}$ & $\begin{array}{l}0 \\
0 \\
0 \\
0 \\
0\end{array}$ & $\begin{array}{l}0 \\
0 \\
0 \\
1 \\
0\end{array}$ & $\frac{U}{U} \frac{U}{2}$ & $\begin{array}{l}U \\
U \\
2 \\
6 \\
-\end{array}$ \\
\hline
\end{tabular}

C.N.M.I.: Commonwealth of Northern Mariana Islands

$\mathrm{U}$ : Unavailable. ․: No reported cases. $\quad \mathrm{N}$ : Not notifiable. Cum: Cumulative year-to-date counts.

Med: Median

Max: Maximum.

* Incidence data for reporting year 2006 is provisional.

${ }^{\dagger}$ Contains data reported through the National Electronic Disease Surveillance System (NEDSS). 
TABLE II. (Continued) Provisional cases of selected notifiable diseases, United States, weeks ending November 11, 2006, and November 12, 2005 (45th Week)*

\begin{tabular}{|c|c|c|c|c|c|c|c|c|c|c|c|c|c|c|c|}
\hline \multirow[b]{3}{*}{ Reporting area } & \multicolumn{5}{|c|}{ Rabies, animal } & \multicolumn{5}{|c|}{ Rocky Mountain spotted fever } & \multicolumn{5}{|c|}{ Salmonellosis } \\
\hline & \multirow{2}{*}{$\begin{array}{c}\text { Current } \\
\text { week }\end{array}$} & \multicolumn{2}{|c|}{$\begin{array}{l}\text { Previous } \\
52 \text { weeks }\end{array}$} & \multirow{2}{*}{$\begin{array}{l}\text { Cum } \\
2006\end{array}$} & \multirow{2}{*}{$\begin{array}{l}\text { Cum } \\
2005\end{array}$} & \multirow{2}{*}{$\begin{array}{c}\text { Current } \\
\text { week }\end{array}$} & \multicolumn{2}{|c|}{$\begin{array}{l}\text { Previous } \\
52 \text { weeks }\end{array}$} & \multirow{2}{*}{$\begin{array}{l}\text { Cum } \\
2006\end{array}$} & \multirow{2}{*}{$\begin{array}{l}\text { Cum } \\
2005\end{array}$} & \multirow{2}{*}{$\begin{array}{c}\text { Current } \\
\text { week }\end{array}$} & \multicolumn{2}{|c|}{$\begin{array}{l}\text { Previous } \\
52 \text { weeks }\end{array}$} & \multirow{2}{*}{$\begin{array}{l}\text { Cum } \\
2006\end{array}$} & \multirow{2}{*}{$\begin{array}{l}\text { Cum } \\
2005 \\
\end{array}$} \\
\hline & & Med & $\operatorname{Max}$ & & & & Med & Max & & & & Med & $\operatorname{Max}$ & & \\
\hline United States & 37 & 119 & 228 & 5,457 & 5,253 & 47 & 38 & 246 & 1,880 & 1,545 & 499 & 800 & 2,291 & 36,038 & 38,454 \\
\hline $\begin{array}{l}\text { New England } \\
\text { Connecticut } \\
\text { Maine }^{\dagger} \\
\text { Massachusetts } \\
\text { New Hampshire } \\
\text { Rhode Island } \\
\text { Vermont }^{\dagger}\end{array}$ & $\begin{array}{r}9 \\
4 \\
- \\
2 \\
1 \\
2\end{array}$ & $\begin{array}{r}11 \\
3 \\
2 \\
4 \\
0 \\
0 \\
1\end{array}$ & $\begin{array}{r}26 \\
14 \\
8 \\
17 \\
5 \\
3 \\
5\end{array}$ & $\begin{array}{r}594 \\
186 \\
98 \\
178 \\
48 \\
24 \\
60\end{array}$ & $\begin{array}{r}634 \\
183 \\
53 \\
306 \\
12 \\
27 \\
53\end{array}$ & $\begin{array}{l}- \\
\bar{N} \\
- \\
-\end{array}$ & $\begin{array}{l}0 \\
0 \\
0 \\
0 \\
0 \\
0 \\
0\end{array}$ & $\begin{array}{l}2 \\
0 \\
0 \\
1 \\
1 \\
2 \\
0\end{array}$ & $\begin{array}{l}2 \\
N \\
1 \\
1 \\
- \\
-\end{array}$ & $\begin{array}{l}8 \\
N \\
6 \\
1 \\
1 \\
-\end{array}$ & $\begin{array}{l}\frac{4}{-} \\
- \\
- \\
-\end{array}$ & $\begin{array}{r}26 \\
0 \\
2 \\
17 \\
3 \\
0 \\
1\end{array}$ & $\begin{array}{r}447 \\
439 \\
10 \\
53 \\
25 \\
17 \\
6\end{array}$ & $\begin{array}{r}1,661 \\
439 \\
102 \\
782 \\
191 \\
83 \\
64\end{array}$ & $\begin{array}{r}1,941 \\
428 \\
151 \\
1,027 \\
154 \\
95 \\
86\end{array}$ \\
\hline $\begin{array}{l}\text { Mid. Atlantic } \\
\text { New Jersey } \\
\text { New York (Upstate) } \\
\text { New York City } \\
\text { Pennsylvania }\end{array}$ & $\begin{array}{r}6 \\
\mathrm{~N} \\
6 \\
- \\
-\end{array}$ & $\begin{array}{r}27 \\
0 \\
11 \\
0 \\
16\end{array}$ & $\begin{array}{r}61 \\
0 \\
24 \\
5 \\
45\end{array}$ & $\begin{array}{r}1,392 \\
N \\
489 \\
27 \\
876\end{array}$ & $\begin{array}{r}883 \\
N \\
495 \\
26 \\
362\end{array}$ & $\frac{3}{1}$ & $\begin{array}{l}1 \\
0 \\
0 \\
0 \\
1\end{array}$ & $\begin{array}{l}5 \\
1 \\
2 \\
3 \\
3\end{array}$ & $\begin{array}{r}70 \\
7 \\
5 \\
17 \\
41\end{array}$ & $\begin{array}{r}91 \\
27 \\
1 \\
7 \\
56\end{array}$ & $\frac{39}{\frac{31}{8}}$ & $\begin{array}{l}83 \\
14 \\
23 \\
23 \\
29\end{array}$ & $\begin{array}{r}272 \\
48 \\
233 \\
48 \\
67\end{array}$ & $\begin{array}{r}4,449 \\
802 \\
1,127 \\
1,071 \\
1,449\end{array}$ & $\begin{array}{r}4,561 \\
892 \\
1,082 \\
1,081 \\
1,506\end{array}$ \\
\hline $\begin{array}{l}\text { E.N. Central } \\
\text { Illinois } \\
\text { Indiana } \\
\text { Michigan } \\
\text { Ohio } \\
\text { Wisconsin }\end{array}$ & $\begin{array}{l}- \\
- \\
- \\
N\end{array}$ & $\begin{array}{l}2 \\
0 \\
0 \\
1 \\
0 \\
0\end{array}$ & $\begin{array}{r}18 \\
7 \\
2 \\
5 \\
9 \\
0\end{array}$ & $\begin{array}{r}152 \\
46 \\
11 \\
44 \\
51 \\
\mathrm{~N}\end{array}$ & $\begin{array}{r}167 \\
50 \\
11 \\
36 \\
70 \\
\mathrm{~N}\end{array}$ & $\begin{array}{l}\frac{1}{-} \\
\frac{1}{-}\end{array}$ & $\begin{array}{l}0 \\
0 \\
0 \\
0 \\
0 \\
0\end{array}$ & $\begin{array}{l}6 \\
1 \\
1 \\
1 \\
4 \\
1\end{array}$ & $\begin{array}{r}35 \\
3 \\
5 \\
2 \\
24 \\
1\end{array}$ & $\begin{array}{r}41 \\
11 \\
1 \\
6 \\
21 \\
2\end{array}$ & $\begin{array}{r}40 \\
18 \\
1 \\
21 \\
-\end{array}$ & $\begin{array}{r}101 \\
24 \\
15 \\
18 \\
22 \\
16\end{array}$ & $\begin{array}{r}187 \\
51 \\
67 \\
34 \\
56 \\
27\end{array}$ & $\begin{array}{r}4,433 \\
991 \\
774 \\
840 \\
1,105 \\
723\end{array}$ & $\begin{array}{r}5,061 \\
1,663 \\
557 \\
817 \\
1,182 \\
842\end{array}$ \\
\hline $\begin{array}{l}\text { W.N. Central } \\
\text { lowa } \\
\text { Kansas } \\
\text { Minnesota } \\
\text { Missouri } \\
\text { Nebraska }^{\dagger} \\
\text { North Dakota } \\
\text { South Dakota }\end{array}$ & $\begin{array}{l}8 \\
1 \\
1 \\
- \\
6 \\
-\end{array}$ & $\begin{array}{l}5 \\
1 \\
1 \\
1 \\
1 \\
0 \\
0 \\
0\end{array}$ & $\begin{array}{r}20 \\
7 \\
5 \\
6 \\
6 \\
0 \\
7 \\
4\end{array}$ & $\begin{array}{r}274 \\
57 \\
71 \\
39 \\
64 \\
22 \\
21\end{array}$ & $\begin{array}{r}299 \\
74 \\
66 \\
68 \\
29 \\
62\end{array}$ & $\begin{array}{l}1 \\
- \\
- \\
- \\
- \\
-\end{array}$ & $\begin{array}{l}2 \\
0 \\
0 \\
0 \\
2 \\
0 \\
0 \\
0\end{array}$ & $\begin{array}{r}15 \\
1 \\
1 \\
2 \\
10 \\
5 \\
1 \\
0\end{array}$ & $\begin{array}{r}198 \\
5 \\
4 \\
4 \\
161 \\
24 \\
- \\
-\end{array}$ & $\begin{array}{r}147 \\
7 \\
5 \\
2 \\
121 \\
7 \\
-5\end{array}$ & $\begin{array}{r}27 \\
2 \\
3 \\
14 \\
5 \\
3 \\
- \\
-\end{array}$ & $\begin{array}{r}44 \\
8 \\
7 \\
11 \\
14 \\
3 \\
0 \\
3\end{array}$ & $\begin{array}{r}107 \\
21 \\
16 \\
60 \\
35 \\
8 \\
46 \\
7\end{array}$ & $\begin{array}{r}2,306 \\
381 \\
322 \\
639 \\
667 \\
162 \\
27 \\
108\end{array}$ & $\begin{array}{r}2,298 \\
378 \\
328 \\
495 \\
719 \\
199 \\
36 \\
143\end{array}$ \\
\hline $\begin{array}{l}\text { S. Atlantic } \\
\text { Delaware } \\
\text { District of Columbia } \\
\text { Florida } \\
\text { Georgia } \\
\text { Maryland }^{\dagger} \\
\text { North Carolina }^{+} \text {South Carolina }^{\dagger} \\
\text { Virginia }^{\dagger} \\
\text { West Virginia }^{\text {West }}\end{array}$ & $\begin{array}{l}10 \\
- \\
- \\
- \\
\frac{9}{-} \\
1\end{array}$ & $\begin{array}{r}38 \\
0 \\
0 \\
0 \\
4 \\
7 \\
9 \\
3 \\
11 \\
1\end{array}$ & $\begin{array}{r}173 \\
0 \\
0 \\
157 \\
9 \\
13 \\
22 \\
11 \\
27 \\
13\end{array}$ & $\begin{array}{r}1,876 \\
- \\
157 \\
189 \\
300 \\
458 \\
154 \\
523 \\
95\end{array}$ & $\begin{array}{r}1,880 \\
- \\
201 \\
235 \\
341 \\
421 \\
196 \\
434 \\
52\end{array}$ & $\begin{array}{l}41 \\
- \\
- \\
- \\
1 \\
- \\
-\end{array}$ & $\begin{array}{r}16 \\
0 \\
0 \\
0 \\
0 \\
1 \\
14 \\
0 \\
1 \\
0\end{array}$ & $\begin{array}{r}94 \\
3 \\
1 \\
3 \\
5 \\
6 \\
87 \\
5 \\
13 \\
2\end{array}$ & $\begin{array}{r}1,069 \\
18 \\
1 \\
19 \\
40 \\
70 \\
795 \\
32 \\
91 \\
3\end{array}$ & $\begin{array}{r}797 \\
7 \\
2 \\
13 \\
85 \\
66 \\
443 \\
67 \\
107 \\
7\end{array}$ & $\begin{array}{r}201 \\
2 \\
123 \\
33 \\
11 \\
29 \\
3 \\
- \\
-\end{array}$ & $\begin{array}{r}218 \\
2 \\
1 \\
95 \\
29 \\
12 \\
34 \\
18 \\
20 \\
2\end{array}$ & $\begin{array}{r}394 \\
10 \\
4 \\
185 \\
75 \\
29 \\
130 \\
51 \\
57 \\
19\end{array}$ & $\begin{array}{r}9,795 \\
136 \\
56 \\
4,152 \\
1,520 \\
620 \\
1,465 \\
873 \\
849 \\
124\end{array}$ & $\begin{array}{r}11,125 \\
114 \\
52 \\
4,545 \\
1,765 \\
734 \\
1,470 \\
1,271 \\
1,007 \\
167\end{array}$ \\
\hline $\begin{array}{l}\text { E.S. Central } \\
\text { Alabama }^{\dagger} \\
\text { Kentucky } \\
\text { Mississippi } \\
\text { Tennessee }^{\dagger}\end{array}$ & $\begin{array}{r}2 \\
2 \\
- \\
-\end{array}$ & $\begin{array}{l}4 \\
1 \\
0 \\
0 \\
2\end{array}$ & $\begin{array}{r}16 \\
8 \\
4 \\
2 \\
9\end{array}$ & $\begin{array}{r}224 \\
78 \\
27 \\
4 \\
115\end{array}$ & $\begin{array}{r}139 \\
74 \\
16 \\
5 \\
44\end{array}$ & $\begin{array}{r}1 \\
1 \\
- \\
-\end{array}$ & $\begin{array}{l}6 \\
1 \\
0 \\
0 \\
4\end{array}$ & $\begin{array}{r}30 \\
10 \\
1 \\
1 \\
21\end{array}$ & $\begin{array}{r}337 \\
108 \\
3 \\
2 \\
224\end{array}$ & $\begin{array}{r}274 \\
69 \\
3 \\
16 \\
186\end{array}$ & $\begin{array}{r}47 \\
43 \\
1 \\
3\end{array}$ & $\begin{array}{r}50 \\
15 \\
8 \\
12 \\
14\end{array}$ & $\begin{array}{r}149 \\
71 \\
23 \\
42 \\
31\end{array}$ & $\begin{array}{r}2,673 \\
956 \\
383 \\
673 \\
661\end{array}$ & $\begin{array}{r}2,663 \\
646 \\
442 \\
828 \\
747\end{array}$ \\
\hline $\begin{array}{l}\text { W.S. Central } \\
\text { Arkansas } \\
\text { Louisiana } \\
\text { Oklahoma } \\
\text { Texas }^{\dagger}\end{array}$ & $\begin{array}{l}- \\
- \\
-\end{array}$ & $\begin{array}{r}13 \\
0 \\
0 \\
1 \\
10\end{array}$ & $\begin{array}{r}34 \\
4 \\
0 \\
9 \\
29\end{array}$ & $\begin{array}{r}555 \\
26 \\
58 \\
471\end{array}$ & $\begin{array}{r}804 \\
33 \\
71 \\
700\end{array}$ & $\begin{array}{l}- \\
- \\
-\end{array}$ & $\begin{array}{l}1 \\
0 \\
0 \\
0 \\
0\end{array}$ & $\begin{array}{r}161 \\
10 \\
1 \\
154 \\
4\end{array}$ & $\begin{array}{r}112 \\
49 \\
4 \\
35 \\
24\end{array}$ & $\begin{array}{r}158 \\
116 \\
6 \\
7 \\
29\end{array}$ & $\begin{array}{r}24 \\
15 \\
1 \\
8 \\
-\end{array}$ & $\begin{array}{r}80 \\
15 \\
13 \\
8 \\
32\end{array}$ & $\begin{array}{r}922 \\
47 \\
42 \\
48 \\
839\end{array}$ & $\begin{array}{r}3,517 \\
834 \\
719 \\
448 \\
1,516\end{array}$ & $\begin{array}{r}3,845 \\
665 \\
831 \\
364 \\
1,985\end{array}$ \\
\hline $\begin{array}{l}\text { Mountain } \\
\text { Arizona } \\
\text { Colorado } \\
\text { Idaho }^{\dagger} \\
\text { Montana }^{\dagger} \\
\text { Nevada }^{\dagger} \\
\text { New Mexico }^{\dagger} \\
\text { Utah } \\
\text { Wyoming }\end{array}$ & $\begin{array}{l}1 \\
- \\
- \\
- \\
- \\
-\end{array}$ & $\begin{array}{l}3 \\
2 \\
0 \\
0 \\
0 \\
0 \\
0 \\
0 \\
0\end{array}$ & $\begin{array}{r}27 \\
10 \\
0 \\
25 \\
2 \\
1 \\
2 \\
1 \\
2\end{array}$ & $\begin{array}{r}196 \\
128 \\
25 \\
13 \\
2 \\
9 \\
11 \\
8\end{array}$ & $\begin{array}{r}248 \\
159 \\
18 \\
15 \\
14 \\
10 \\
15 \\
17\end{array}$ & $\begin{array}{l}- \\
- \\
- \\
- \\
-\end{array}$ & $\begin{array}{l}1 \\
0 \\
0 \\
0 \\
0 \\
0 \\
0 \\
0 \\
0\end{array}$ & $\begin{array}{l}6 \\
6 \\
1 \\
3 \\
2 \\
0 \\
2 \\
2 \\
1\end{array}$ & $\begin{array}{r}50 \\
12 \\
2 \\
13 \\
2 \\
8 \\
6 \\
7\end{array}$ & $\begin{array}{r}27 \\
13 \\
4 \\
3 \\
1 \\
4 \\
2\end{array}$ & $\begin{array}{l}8 \\
6 \\
2 \\
- \\
- \\
- \\
-\end{array}$ & $\begin{array}{r}53 \\
17 \\
12 \\
3 \\
3 \\
3 \\
4 \\
5 \\
1\end{array}$ & $\begin{array}{r}87 \\
67 \\
30 \\
9 \\
16 \\
20 \\
15 \\
15 \\
4\end{array}$ & $\begin{array}{r}2,227 \\
742 \\
548 \\
156 \\
112 \\
171 \\
212 \\
245 \\
41\end{array}$ & $\begin{array}{r}2,093 \\
585 \\
515 \\
130 \\
99 \\
172 \\
228 \\
284 \\
80\end{array}$ \\
\hline $\begin{array}{l}\text { Pacific } \\
\text { Alaska } \\
\text { California } \\
\text { Hawaii } \\
\text { Oregon }{ }^{\dagger} \\
\text { Washington }^{-}\end{array}$ & $\begin{array}{l}\frac{1}{1} \\
- \\
\end{array}$ & $\begin{array}{l}4 \\
0 \\
3 \\
0 \\
0 \\
0\end{array}$ & $\begin{array}{r}10 \\
4 \\
9 \\
0 \\
4 \\
0\end{array}$ & $\begin{array}{r}194 \\
15 \\
159 \\
20 \\
U\end{array}$ & $\begin{array}{r}199 \\
1 \\
191 \\
7 \\
\end{array}$ & $\begin{array}{l}- \\
- \\
- \\
N\end{array}$ & $\begin{array}{l}0 \\
0 \\
0 \\
0 \\
0 \\
0\end{array}$ & $\begin{array}{l}1 \\
0 \\
1 \\
0 \\
1 \\
0\end{array}$ & $\begin{array}{l}\frac{7}{5} \\
\frac{5}{2} \\
\mathrm{~N}\end{array}$ & $\begin{array}{l}\frac{2}{-} \\
\frac{-}{2} \\
\mathrm{~N}\end{array}$ & $\begin{array}{r}109 \\
106 \\
3 \\
- \\
-\end{array}$ & $\begin{array}{r}109 \\
1 \\
88 \\
5 \\
7 \\
9\end{array}$ & $\begin{array}{r}426 \\
7 \\
292 \\
10 \\
16 \\
124\end{array}$ & $\begin{array}{r}4,977 \\
66 \\
3,917 \\
211 \\
343 \\
440\end{array}$ & $\begin{array}{r}4,867 \\
51 \\
3,719 \\
264 \\
365 \\
468\end{array}$ \\
\hline $\begin{array}{l}\text { American Samoa } \\
\text { C.N.M.I. } \\
\text { Guam } \\
\text { Puerto Rico } \\
\text { U.S. Virgin Islands }\end{array}$ & $\begin{array}{l}U \\
\underline{U} \\
- \\
-\end{array}$ & $\begin{array}{l}0 \\
0 \\
0 \\
1 \\
0\end{array}$ & $\begin{array}{l}0 \\
0 \\
0 \\
6 \\
0\end{array}$ & $\frac{U}{\frac{U}{68}}$ & $\frac{U}{U} \frac{U}{60}$ & $\frac{U}{U} \frac{U}{N}$ & $\begin{array}{l}0 \\
0 \\
0 \\
0 \\
0\end{array}$ & $\begin{array}{l}0 \\
0 \\
0 \\
0 \\
0\end{array}$ & $\frac{U}{U} \frac{U}{-}$ & $\frac{U}{U}$ & $\begin{array}{l}U \\
\underline{U} \\
- \\
-\end{array}$ & $\begin{array}{l}0 \\
0 \\
1 \\
4 \\
0\end{array}$ & $\begin{array}{r}0 \\
0 \\
3 \\
35 \\
0\end{array}$ & $\frac{U}{U} \frac{U}{199}$ & $\begin{array}{r}7 \\
U \\
34 \\
561 \\
-\end{array}$ \\
\hline
\end{tabular}

C.N.M.I.: Commonwealth of Northern Mariana Islands.

U: Unavailable. ․: No reported cases. N: Not notifiable. Cum: Cumulative year-to-date counts. Med: Median. Max: Maximum.

* Incidence data for reporting year 2006 is provisional.

${ }^{\dagger}$ Contains data reported through the National Electronic Disease Surveillance System (NEDSS). 
TABLE II. (Continued) Provisional cases of selected notifiable diseases, United States, weeks ending November 11, 2006, and November 12, 2005 (45th Week)*

\begin{tabular}{|c|c|c|c|c|c|c|c|c|c|c|c|c|c|c|c|}
\hline \multirow[b]{3}{*}{ Reporting area } & \multicolumn{5}{|c|}{ Shiga toxin-producing E. coli (STEC) ${ }^{\dagger}$} & \multicolumn{5}{|c|}{ Shigellosis } & \multicolumn{5}{|c|}{ Streptococcal disease, invasive, group A } \\
\hline & \multirow{2}{*}{$\begin{array}{c}\text { Current } \\
\text { week }\end{array}$} & \multicolumn{2}{|c|}{$\begin{array}{l}\text { Previous } \\
52 \text { weeks } \\
\end{array}$} & \multirow{2}{*}{$\begin{array}{l}\text { Cum } \\
2006\end{array}$} & \multirow{2}{*}{$\begin{array}{l}\text { Cum } \\
2005\end{array}$} & \multirow{2}{*}{$\begin{array}{c}\text { Current } \\
\text { week }\end{array}$} & \multicolumn{2}{|c|}{$\begin{array}{l}\text { Previous } \\
52 \text { weeks } \\
\end{array}$} & \multirow{2}{*}{$\begin{array}{l}\text { Cum } \\
2006\end{array}$} & \multirow{2}{*}{$\begin{array}{l}\text { Cum } \\
2005\end{array}$} & \multirow{2}{*}{$\begin{array}{c}\text { Current } \\
\text { week }\end{array}$} & \multicolumn{2}{|c|}{$\begin{array}{l}\text { Previous } \\
52 \text { weeks } \\
\end{array}$} & \multirow{2}{*}{$\begin{array}{l}\text { Cum } \\
2006\end{array}$} & \multirow{2}{*}{$\begin{array}{l}\text { Cum } \\
2005\end{array}$} \\
\hline & & Med & Max & & & & Med & Max & & & & Med & Max & & \\
\hline United States & 23 & 52 & 297 & 2,405 & 2,883 & 310 & 256 & 1,013 & 11,376 & 13,243 & 41 & 93 & 282 & 4,169 & 3,924 \\
\hline $\begin{array}{l}\text { New England } \\
\text { Connecticut } \\
\text { Maine§ } \\
\text { Massachusetts } \\
\text { New Hampshire } \\
\text { Rhode Island } \\
\text { Vermont }{ }^{\S}\end{array}$ & $\begin{array}{l}- \\
- \\
- \\
-\end{array}$ & $\begin{array}{l}3 \\
0 \\
0 \\
1 \\
0 \\
0 \\
0\end{array}$ & $\begin{array}{r}70 \\
69 \\
8 \\
9 \\
3 \\
2 \\
2\end{array}$ & $\begin{array}{r}232 \\
69 \\
31 \\
82 \\
24 \\
8 \\
2\end{array}$ & $\begin{array}{r}201 \\
52 \\
28 \\
82 \\
15 \\
7 \\
17\end{array}$ & $\begin{array}{l}- \\
- \\
- \\
-\end{array}$ & $\begin{array}{l}3 \\
0 \\
0 \\
2 \\
0 \\
0 \\
0\end{array}$ & $\begin{array}{r}65 \\
59 \\
2 \\
11 \\
4 \\
3 \\
2\end{array}$ & $\begin{array}{r}216 \\
59 \\
3 \\
128 \\
7 \\
13 \\
6\end{array}$ & $\begin{array}{r}288 \\
51 \\
14 \\
174 \\
13 \\
20 \\
16\end{array}$ & $\begin{array}{l}\bar{U} \\
- \\
- \\
-\end{array}$ & $\begin{array}{l}4 \\
0 \\
0 \\
2 \\
0 \\
0 \\
0\end{array}$ & $\begin{array}{r}15 \\
2 \\
2 \\
6 \\
9 \\
3 \\
2\end{array}$ & $\begin{array}{r}182 \\
U \\
17 \\
101 \\
44 \\
7 \\
13\end{array}$ & $\begin{array}{r}257 \\
91 \\
14 \\
116 \\
17 \\
9 \\
10\end{array}$ \\
\hline $\begin{array}{l}\text { Mid. Atlantic } \\
\text { New Jersey } \\
\text { New York (Upstate) } \\
\text { New York City } \\
\text { Pennsylvania }\end{array}$ & $\begin{array}{l}1 \\
- \\
-\end{array}$ & $\begin{array}{l}4 \\
0 \\
0 \\
0 \\
0\end{array}$ & $\begin{array}{r}107 \\
3 \\
103 \\
4 \\
4\end{array}$ & $\begin{array}{r}183 \\
3 \\
12 \\
32 \\
8\end{array}$ & $\begin{array}{r}327 \\
69 \\
124 \\
17 \\
117\end{array}$ & $\frac{1}{-}$ & $\begin{array}{r}16 \\
4 \\
4 \\
5 \\
1\end{array}$ & $\begin{array}{r}72 \\
34 \\
60 \\
12 \\
6\end{array}$ & $\begin{array}{r}738 \\
241 \\
201 \\
219 \\
77\end{array}$ & $\begin{array}{r}1,122 \\
284 \\
240 \\
371 \\
227\end{array}$ & $\frac{8}{\frac{8}{5}}$ & $\begin{array}{r}18 \\
2 \\
4 \\
3 \\
6\end{array}$ & $\begin{array}{r}43 \\
8 \\
32 \\
8 \\
13\end{array}$ & $\begin{array}{l}800 \\
122 \\
268 \\
133 \\
277\end{array}$ & $\begin{array}{l}777 \\
164 \\
217 \\
151 \\
245\end{array}$ \\
\hline $\begin{array}{l}\text { E.N. Central } \\
\text { Illinois } \\
\text { Indiana } \\
\text { Michigan } \\
\text { Ohio } \\
\text { Wisconsin }\end{array}$ & $\begin{array}{l}\frac{5}{-} \\
\frac{5}{-}\end{array}$ & $\begin{array}{r}10 \\
1 \\
1 \\
2 \\
2 \\
2\end{array}$ & $\begin{array}{r}54 \\
7 \\
8 \\
7 \\
18 \\
39\end{array}$ & $\begin{array}{r}554 \\
64 \\
76 \\
80 \\
162 \\
172\end{array}$ & $\begin{array}{r}575 \\
126 \\
64 \\
82 \\
153 \\
150\end{array}$ & $\frac{\frac{16}{10}}{\frac{6}{-}}$ & $\begin{array}{r}20 \\
7 \\
2 \\
3 \\
3 \\
3\end{array}$ & $\begin{array}{r}37 \\
18 \\
18 \\
8 \\
14 \\
9\end{array}$ & $\begin{array}{l}886 \\
307 \\
142 \\
133 \\
172 \\
132\end{array}$ & $\begin{array}{r}1,028 \\
354 \\
153 \\
213 \\
101 \\
207\end{array}$ & $\begin{array}{l}\frac{2}{1} \\
\frac{1}{-}\end{array}$ & $\begin{array}{r}14 \\
3 \\
2 \\
3 \\
4 \\
1\end{array}$ & $\begin{array}{r}43 \\
11 \\
11 \\
12 \\
19 \\
4\end{array}$ & $\begin{array}{r}704 \\
144 \\
101 \\
193 \\
215 \\
51\end{array}$ & $\begin{array}{r}805 \\
270 \\
92 \\
188 \\
171 \\
84\end{array}$ \\
\hline $\begin{array}{l}\text { W.N. Central } \\
\text { lowa } \\
\text { Kansas } \\
\text { Minnesota } \\
\text { Missouri } \\
\text { Nebraska } \\
\text { North Dakota } \\
\text { South Dakota }\end{array}$ & $\begin{array}{l}8 \\
-7 \\
-1 \\
- \\
-\end{array}$ & $\begin{array}{l}8 \\
2 \\
0 \\
3 \\
1 \\
1 \\
0 \\
0\end{array}$ & $\begin{array}{r}32 \\
8 \\
4 \\
27 \\
10 \\
8 \\
15 \\
5\end{array}$ & $\begin{array}{r}476 \\
116 \\
21 \\
218 \\
82 \\
55 \\
40\end{array}$ & $\begin{array}{r}487 \\
94 \\
51 \\
160 \\
89 \\
56 \\
7 \\
30\end{array}$ & $\begin{array}{l}15 \\
- \\
9 \\
4 \\
-2 \\
-\end{array}$ & $\begin{array}{r}35 \\
2 \\
3 \\
2 \\
11 \\
2 \\
0 \\
4\end{array}$ & $\begin{array}{l}77 \\
10 \\
20 \\
23 \\
69 \\
14 \\
18 \\
22\end{array}$ & $\begin{array}{r}1,472 \\
94 \\
128 \\
201 \\
604 \\
118 \\
103 \\
224\end{array}$ & $\begin{array}{r}1,462 \\
90 \\
205 \\
81 \\
877 \\
123 \\
4 \\
82\end{array}$ & $\begin{array}{l}7 \\
\mathrm{~N} \\
-7 \\
- \\
- \\
- \\
-\end{array}$ & $\begin{array}{l}5 \\
0 \\
1 \\
0 \\
1 \\
0 \\
0 \\
0\end{array}$ & $\begin{array}{r}57 \\
0 \\
5 \\
52 \\
5 \\
4 \\
5 \\
3\end{array}$ & $\begin{array}{r}304 \\
\mathrm{~N} \\
52 \\
143 \\
63 \\
27 \\
11 \\
8\end{array}$ & $\begin{array}{r}240 \\
\mathrm{~N} \\
36 \\
90 \\
61 \\
22 \\
10 \\
21\end{array}$ \\
\hline $\begin{array}{l}\text { S. Atlantic } \\
\text { Delaware } \\
\text { District of Columbia } \\
\text { Florida } \\
\text { Georgia } \\
\text { Maryland§ } \\
\text { North Carolina } \\
\text { South Carolina } \\
\text { Virginia } \\
\text { West Virginia }\end{array}$ & $\begin{array}{l}5 \\
-1 \\
2 \\
2 \\
1 \\
- \\
-\end{array}$ & $\begin{array}{l}8 \\
0 \\
0 \\
2 \\
1 \\
1 \\
2 \\
0 \\
0 \\
0\end{array}$ & $\begin{array}{r}39 \\
2 \\
1 \\
29 \\
6 \\
8 \\
7 \\
2 \\
8 \\
5\end{array}$ & $\begin{array}{r}404 \\
7 \\
2 \\
82 \\
79 \\
83 \\
101 \\
8 \\
\frac{1}{12}\end{array}$ & $\begin{array}{r}369 \\
9 \\
1 \\
82 \\
48 \\
70 \\
58 \\
11 \\
87 \\
3\end{array}$ & $\begin{array}{r}118 \\
- \\
52 \\
61 \\
1 \\
4 \\
- \\
-\end{array}$ & $\begin{array}{r}58 \\
0 \\
0 \\
27 \\
19 \\
2 \\
1 \\
1 \\
1 \\
0\end{array}$ & $\begin{array}{r}124 \\
2 \\
2 \\
77 \\
42 \\
10 \\
21 \\
9 \\
9 \\
2\end{array}$ & $\begin{array}{r}2,784 \\
9 \\
15 \\
1,343 \\
1,005 \\
112 \\
143 \\
72 \\
81 \\
4\end{array}$ & $\begin{array}{r}2,095 \\
11 \\
12 \\
1,018 \\
576 \\
92 \\
179 \\
92 \\
114 \\
1\end{array}$ & $\begin{array}{l}16 \\
- \\
8 \\
6 \\
2 \\
- \\
- \\
-\end{array}$ & $\begin{array}{r}22 \\
0 \\
0 \\
5 \\
5 \\
4 \\
0 \\
1 \\
2 \\
0\end{array}$ & $\begin{array}{r}44 \\
2 \\
2 \\
16 \\
12 \\
12 \\
26 \\
6 \\
11 \\
6\end{array}$ & $\begin{array}{r}1,009 \\
10 \\
15 \\
264 \\
205 \\
177 \\
145 \\
54 \\
113 \\
26\end{array}$ & $\begin{array}{r}811 \\
6 \\
10 \\
215 \\
175 \\
157 \\
115 \\
32 \\
79 \\
22\end{array}$ \\
\hline $\begin{array}{l}\text { E.S. Central } \\
\text { Alabama§ } \\
\text { Kentucky } \\
\text { Mississippi } \\
\text { Tennessee }^{\S}\end{array}$ & $\begin{array}{l}-1 \\
- \\
-\end{array}$ & $\begin{array}{l}1 \\
0 \\
1 \\
0 \\
0\end{array}$ & $\begin{array}{r}12 \\
5 \\
12 \\
0 \\
4\end{array}$ & $\begin{array}{l}89 \\
39 \\
89 \\
-24\end{array}$ & $\begin{array}{r}168 \\
28 \\
72 \\
8 \\
60\end{array}$ & $\begin{array}{l}73 \\
71 \\
- \\
2\end{array}$ & $\begin{array}{r}13 \\
3 \\
4 \\
1 \\
2\end{array}$ & $\begin{array}{r}50 \\
31 \\
15 \\
8 \\
12\end{array}$ & $\begin{array}{r}761 \\
333 \\
211 \\
76 \\
141\end{array}$ & $\begin{array}{r}1,095 \\
207 \\
285 \\
85 \\
518\end{array}$ & $\begin{array}{l}2 \\
\mathrm{~N} \\
- \\
2\end{array}$ & $\begin{array}{l}3 \\
0 \\
0 \\
0 \\
3\end{array}$ & $\begin{array}{r}11 \\
0 \\
5 \\
0 \\
9\end{array}$ & $\begin{array}{r}177 \\
N \\
34 \\
\frac{143}{}\end{array}$ & $\begin{array}{r}156 \\
N \\
31 \\
-125\end{array}$ \\
\hline $\begin{array}{l}\text { W.S. Central } \\
\text { Arkansas } \\
\text { Louisiana } \\
\text { Oklahoma } \\
\text { Texas }^{\S}\end{array}$ & $\frac{-}{-}$ & $\begin{array}{l}1 \\
0 \\
0 \\
0 \\
2\end{array}$ & $\begin{array}{r}52 \\
7 \\
1 \\
17 \\
44\end{array}$ & $\begin{array}{l}68 \\
33 \\
-35 \\
97\end{array}$ & $\begin{array}{l}99 \\
12 \\
21 \\
25 \\
41\end{array}$ & $\begin{array}{r}\frac{42}{-} \\
\frac{2}{40}\end{array}$ & $\begin{array}{r}37 \\
2 \\
1 \\
3 \\
29\end{array}$ & $\begin{array}{r}596 \\
9 \\
25 \\
286 \\
308\end{array}$ & $\begin{array}{r}1,553 \\
102 \\
127 \\
119 \\
1,205\end{array}$ & $\begin{array}{r}3,188 \\
56 \\
128 \\
588 \\
2,416\end{array}$ & $\frac{2}{-}$ & $\begin{array}{l}7 \\
0 \\
0 \\
2 \\
4\end{array}$ & $\begin{array}{r}58 \\
5 \\
2 \\
14 \\
43\end{array}$ & $\begin{array}{r}327 \\
25 \\
8 \\
92 \\
202\end{array}$ & $\begin{array}{r}274 \\
19 \\
- \\
101 \\
154\end{array}$ \\
\hline $\begin{array}{l}\text { Mountain } \\
\text { Arizona } \\
\text { Colorado } \\
\text { Idaho } \\
\text { Montana } \\
\text { Nevada }^{\S} \\
\text { New Mexico§ } \\
\text { Utah } \\
\text { Wyoming }\end{array}$ & $\begin{array}{l}3 \\
-2 \\
- \\
- \\
- \\
-\end{array}$ & $\begin{array}{l}5 \\
2 \\
1 \\
1 \\
0 \\
0 \\
0 \\
1 \\
0\end{array}$ & $\begin{array}{r}16 \\
13 \\
8 \\
7 \\
1 \\
5 \\
1 \\
14 \\
3\end{array}$ & $\begin{array}{r}277 \\
109 \\
94 \\
73 \\
-22 \\
4 \\
111 \\
18\end{array}$ & $\begin{array}{r}281 \\
30 \\
73 \\
46 \\
15 \\
21 \\
24 \\
63 \\
9\end{array}$ & $\begin{array}{r}9 \\
1 \\
5 \\
- \\
- \\
- \\
- \\
3\end{array}$ & $\begin{array}{r}23 \\
13 \\
3 \\
0 \\
0 \\
1 \\
2 \\
1 \\
0\end{array}$ & $\begin{array}{r}88 \\
35 \\
16 \\
3 \\
10 \\
20 \\
15 \\
6 \\
8\end{array}$ & $\begin{array}{r}1,231 \\
628 \\
211 \\
14 \\
30 \\
103 \\
152 \\
71 \\
22\end{array}$ & $\begin{array}{r}824 \\
433 \\
148 \\
17 \\
5 \\
55 \\
122 \\
39 \\
5\end{array}$ & $\begin{array}{r}4 \\
-4 \\
- \\
- \\
- \\
- \\
-\end{array}$ & $\begin{array}{r}11 \\
6 \\
3 \\
0 \\
0 \\
0 \\
1 \\
1 \\
0\end{array}$ & $\begin{array}{r}77 \\
57 \\
8 \\
2 \\
0 \\
0 \\
7 \\
7 \\
1\end{array}$ & $\begin{array}{r}567 \\
299 \\
128 \\
8 \\
- \\
66 \\
62 \\
4\end{array}$ & $\begin{array}{r}507 \\
218 \\
157 \\
3 \\
- \\
71 \\
54 \\
4\end{array}$ \\
\hline $\begin{array}{l}\text { Pacific } \\
\text { Alaska } \\
\text { California } \\
\text { Hawaii } \\
\text { Oregon } § \\
\text { Washington }\end{array}$ & $\begin{array}{l}\frac{1}{-} \\
\frac{1}{-}\end{array}$ & $\begin{array}{l}2 \\
0 \\
2 \\
0 \\
2 \\
2\end{array}$ & $\begin{array}{r}50 \\
0 \\
18 \\
2 \\
13 \\
32\end{array}$ & $\begin{array}{r}122 \\
- \\
16 \\
107 \\
106\end{array}$ & $\begin{array}{r}376 \\
127 \\
13 \\
149 \\
87\end{array}$ & $\begin{array}{l}\frac{36}{36} \\
- \\
-\end{array}$ & $\begin{array}{r}38 \\
0 \\
31 \\
1 \\
1 \\
2\end{array}$ & $\begin{array}{r}148 \\
2 \\
104 \\
4 \\
31 \\
43\end{array}$ & $\begin{array}{r}1,735 \\
9 \\
1,440 \\
42 \\
112 \\
132 \\
\end{array}$ & \begin{tabular}{r|}
2,141 \\
11 \\
1,849 \\
31 \\
117 \\
133
\end{tabular} & $\begin{array}{l}- \\
- \\
\bar{N} \\
\mathrm{~N}\end{array}$ & $\begin{array}{l}2 \\
0 \\
0 \\
2 \\
0 \\
0\end{array}$ & $\begin{array}{l}9 \\
0 \\
0 \\
9 \\
0 \\
0\end{array}$ & $\begin{array}{r}99 \\
- \\
99 \\
N \\
N\end{array}$ & $\begin{array}{l}97 \\
- \\
97 \\
N \\
N\end{array}$ \\
\hline $\begin{array}{l}\text { American Samoa } \\
\text { C.N.M.I. } \\
\text { Guam } \\
\text { Puerto Rico } \\
\text { U.S. Virgin Islands }\end{array}$ & $\begin{array}{l}U \\
U \\
- \\
-\end{array}$ & $\begin{array}{l}0 \\
0 \\
0 \\
0 \\
0\end{array}$ & $\begin{array}{l}0 \\
0 \\
0 \\
0 \\
0\end{array}$ & $\begin{array}{l}U \\
U \\
- \\
-\end{array}$ & $\frac{U}{U} \frac{U}{2}$ & $\begin{array}{l}U \\
U \\
- \\
-\end{array}$ & $\begin{array}{l}0 \\
0 \\
0 \\
0 \\
0\end{array}$ & $\begin{array}{l}0 \\
0 \\
3 \\
2 \\
0\end{array}$ & $\frac{U}{U} \frac{U}{12}$ & $\begin{array}{r}7 \\
U \\
16 \\
8 \\
-\end{array}$ & $\begin{array}{l}U \\
\frac{U}{N} \\
-\end{array}$ & $\begin{array}{l}0 \\
0 \\
0 \\
0 \\
0\end{array}$ & $\begin{array}{l}0 \\
0 \\
0 \\
0 \\
0\end{array}$ & $\begin{array}{l}U \\
\frac{U}{N} \\
-\end{array}$ & $\begin{array}{l}U \\
\frac{U}{N} \\
-\end{array}$ \\
\hline
\end{tabular}

C.N.M.I.: Commonwealth of Northern Mariana Islands.

U: Unavailable. $\quad$-: No reported cases. $\quad \mathrm{N}$ : Not notifiable. $\quad$ Cum: Cumulative year-to-date counts.

Med: Median.

Max: Maximum.

* Incidence data for reporting year 2006 is provisional.

† Includes E. coli O157:H7; Shiga toxin positive, serogroup non-0157; and Shiga toxin positive, not serogrouped.

$\S$ Contains data reported through the National Electronic Disease Surveillance System (NEDSS). 
TABLE II. (Continued) Provisional cases of selected notifiable diseases, United States, weeks ending November 11, 2006, and November 12, 2005 (45th Week)*

\begin{tabular}{|c|c|c|c|c|c|c|c|c|c|c|c|c|c|c|c|}
\hline \multirow[b]{3}{*}{ Reporting area } & \multicolumn{5}{|c|}{$\begin{array}{c}\text { Streptococcus pneumoniae, invasive disease } \\
\text { Drug resistant, all ages }\end{array}$} & \multicolumn{5}{|c|}{ Syphilis, primary and secondary } & \multicolumn{5}{|c|}{ Varicella (chickenpox) } \\
\hline & \multirow{2}{*}{$\begin{array}{c}\text { Current } \\
\text { week }\end{array}$} & \multicolumn{2}{|c|}{$\begin{array}{l}\text { Previous } \\
52 \text { weeks }\end{array}$} & \multirow{2}{*}{$\begin{array}{l}\text { Cum } \\
2006\end{array}$} & \multirow{2}{*}{$\begin{array}{l}\text { Cum } \\
2005\end{array}$} & \multirow{2}{*}{$\begin{array}{c}\text { Current } \\
\text { week }\end{array}$} & \multicolumn{2}{|c|}{$\begin{array}{l}\text { Previous } \\
52 \text { weeks }\end{array}$} & \multirow{2}{*}{$\begin{array}{l}\text { Cum } \\
2006\end{array}$} & \multirow{2}{*}{$\begin{array}{l}\text { Cum } \\
2005\end{array}$} & \multirow{2}{*}{$\begin{array}{c}\text { Current } \\
\text { week }\end{array}$} & \multicolumn{2}{|c|}{$\begin{array}{l}\text { Previous } \\
52 \text { weeks }\end{array}$} & \multirow{2}{*}{$\begin{array}{l}\text { Cum } \\
2006\end{array}$} & \multirow{2}{*}{$\begin{array}{l}\text { Cum } \\
2005\end{array}$} \\
\hline & & Med & Max & & & & Med & $\operatorname{Max}$ & & & & Med & $\operatorname{Max}$ & & \\
\hline United States & 33 & 51 & 333 & 2,141 & 2,184 & 88 & 176 & 334 & 7,773 & 7,369 & 469 & 812 & 2,857 & 35,224 & 24,574 \\
\hline $\begin{array}{l}\text { New England } \\
\text { Connecticut } \\
\text { Maine }^{\dagger} \\
\text { Massachusetts } \\
\text { New Hampshire } \\
\text { Rhode Island } \\
\text { Vermont }^{\dagger}\end{array}$ & $\begin{array}{l}1 \\
\frac{U}{-} \\
- \\
-\end{array}$ & $\begin{array}{l}1 \\
0 \\
0 \\
0 \\
0 \\
0 \\
0\end{array}$ & $\begin{array}{r}24 \\
7 \\
2 \\
6 \\
0 \\
11 \\
2\end{array}$ & $\begin{array}{r}32 \\
U \\
8 \\
- \\
10 \\
14\end{array}$ & $\begin{array}{r}197 \\
81 \\
\mathrm{~N} \\
87 \\
18 \\
11\end{array}$ & $\begin{array}{l}1 \\
1 \\
- \\
- \\
-\end{array}$ & $\begin{array}{l}4 \\
0 \\
0 \\
3 \\
0 \\
0 \\
0\end{array}$ & $\begin{array}{r}17 \\
11 \\
2 \\
6 \\
2 \\
2 \\
1\end{array}$ & $\begin{array}{r}174 \\
38 \\
8 \\
106 \\
11 \\
9 \\
2\end{array}$ & $\begin{array}{r}183 \\
42 \\
1 \\
105 \\
13 \\
21 \\
1\end{array}$ & $\frac{21}{\frac{U}{7}}$ & $\begin{array}{r}36 \\
0 \\
4 \\
0 \\
6 \\
0 \\
12\end{array}$ & $\begin{array}{r}144 \\
58 \\
20 \\
54 \\
47 \\
0 \\
50\end{array}$ & $\begin{array}{r}1,284 \\
U \\
151 \\
94 \\
434 \\
605\end{array}$ & $\begin{array}{r}4,569 \\
1,396 \\
267 \\
2,024 \\
279 \\
-603\end{array}$ \\
\hline $\begin{array}{l}\text { Mid. Atlantic } \\
\text { New Jersey } \\
\text { New York (Upstate) } \\
\text { New York City } \\
\text { Pennsylvania }\end{array}$ & $\begin{array}{r}4 \\
N \\
1 \\
U \\
3\end{array}$ & $\begin{array}{l}3 \\
0 \\
1 \\
0 \\
2\end{array}$ & $\begin{array}{r}15 \\
0 \\
10 \\
0 \\
9\end{array}$ & $\begin{array}{r}148 \\
N \\
54 \\
U \\
94\end{array}$ & $\begin{array}{r}182 \\
N \\
71 \\
U \\
111\end{array}$ & $\begin{array}{l}5 \\
2 \\
1 \\
2\end{array}$ & $\begin{array}{r}21 \\
3 \\
3 \\
10 \\
5\end{array}$ & $\begin{array}{r}35 \\
8 \\
14 \\
23 \\
12\end{array}$ & $\begin{array}{l}970 \\
145 \\
132 \\
468 \\
225\end{array}$ & $\begin{array}{r}889 \\
115 \\
69 \\
534 \\
171\end{array}$ & $\frac{60}{-}$ & $\begin{array}{r}102 \\
0 \\
0 \\
0 \\
102\end{array}$ & $\begin{array}{r}183 \\
0 \\
0 \\
0 \\
183\end{array}$ & $\begin{array}{r}\frac{4,128}{-} \\
\frac{-}{4,128}\end{array}$ & $\frac{4,152}{-}$ \\
\hline $\begin{array}{l}\text { E.N. Central } \\
\text { Illinois } \\
\text { Indiana } \\
\text { Michigan } \\
\text { Ohio } \\
\text { Wisconsin }\end{array}$ & $\begin{array}{l}\frac{11}{3} \\
\frac{-}{8} \\
\mathrm{~N}\end{array}$ & $\begin{array}{r}12 \\
0 \\
2 \\
0 \\
6 \\
0\end{array}$ & $\begin{array}{r}41 \\
3 \\
21 \\
4 \\
32 \\
0\end{array}$ & $\begin{array}{r}502 \\
17 \\
140 \\
18 \\
327 \\
\mathrm{~N}\end{array}$ & $\begin{array}{r}546 \\
30 \\
166 \\
38 \\
312 \\
\mathrm{~N}\end{array}$ & $\begin{array}{r}11 \\
1 \\
1 \\
3 \\
4 \\
2\end{array}$ & $\begin{array}{r}17 \\
8 \\
1 \\
2 \\
3 \\
1\end{array}$ & $\begin{array}{r}39 \\
23 \\
4 \\
19 \\
8 \\
4\end{array}$ & $\begin{array}{r}763 \\
355 \\
78 \\
105 \\
167 \\
58\end{array}$ & $\begin{array}{r}796 \\
447 \\
55 \\
72 \\
190 \\
32\end{array}$ & $\begin{array}{r}184 \\
- \\
56 \\
128 \\
-\end{array}$ & $\begin{array}{r}234 \\
1 \\
0 \\
102 \\
122 \\
13\end{array}$ & $\begin{array}{r}587 \\
7 \\
475 \\
174 \\
420 \\
52\end{array}$ & $\begin{array}{r}12,498 \\
68 \\
475 \\
3,851 \\
7,460 \\
644\end{array}$ & $\begin{array}{r}4,916 \\
87 \\
- \\
3,155 \\
1,293 \\
381\end{array}$ \\
\hline $\begin{array}{l}\text { W.N. Central } \\
\text { lowa } \\
\text { Kansas } \\
\text { Minnesota } \\
\text { Missouri } \\
\text { Nebraska } \\
\text { North Dakota } \\
\text { South Dakota }\end{array}$ & $\begin{array}{l}1 \\
N \\
N \\
-1 \\
- \\
-\end{array}$ & $\begin{array}{l}1 \\
0 \\
0 \\
0 \\
1 \\
0 \\
0 \\
0\end{array}$ & $\begin{array}{r}191 \\
0 \\
0 \\
191 \\
3 \\
1 \\
1 \\
1\end{array}$ & $\begin{array}{r}99 \\
\mathrm{~N} \\
\mathrm{~N} \\
60 \\
37 \\
1 \\
-1\end{array}$ & $\begin{array}{r}39 \\
\mathrm{~N} \\
\mathrm{~N} \\
32 \\
2 \\
2 \\
3\end{array}$ & $\begin{array}{l}1 \\
-1 \\
- \\
- \\
-\end{array}$ & $\begin{array}{l}5 \\
0 \\
0 \\
0 \\
3 \\
0 \\
0 \\
0\end{array}$ & $\begin{array}{r}11 \\
2 \\
3 \\
2 \\
8 \\
1 \\
1 \\
3\end{array}$ & $\begin{array}{r}218 \\
16 \\
22 \\
21 \\
143 \\
3 \\
1 \\
12\end{array}$ & $\begin{array}{r}228 \\
8 \\
17 \\
65 \\
132 \\
4 \\
1 \\
1\end{array}$ & $\begin{array}{r}48 \\
N \\
6 \\
- \\
42 \\
- \\
- \\
-\end{array}$ & $\begin{array}{r}27 \\
0 \\
3 \\
0 \\
22 \\
0 \\
0 \\
1\end{array}$ & $\begin{array}{r}98 \\
0 \\
24 \\
0 \\
82 \\
0 \\
0 \\
25 \\
12\end{array}$ & $\begin{array}{r}1,499 \\
\mathrm{~N} \\
284 \\
- \\
1,112 \\
- \\
45 \\
58\end{array}$ & $\begin{array}{r}450 \\
\mathrm{~N} \\
- \\
305 \\
- \\
31 \\
114\end{array}$ \\
\hline $\begin{array}{l}\text { S. Atlantic } \\
\text { Delaware } \\
\text { District of Columbia } \\
\text { Florida } \\
\text { Georgia } \\
\text { Maryland }^{\dagger} \\
\text { North Carolina } \\
\text { South Carolina }^{\dagger} \\
\text { Virginia }^{\dagger} \\
\text { West Virginia }\end{array}$ & $\begin{array}{r}\frac{16}{-} \\
\frac{13}{3} \\
\frac{\mathrm{N}}{\mathrm{N}} \\
-\end{array}$ & $\begin{array}{r}26 \\
0 \\
0 \\
14 \\
8 \\
0 \\
0 \\
0 \\
0 \\
1\end{array}$ & $\begin{array}{r}53 \\
2 \\
3 \\
36 \\
29 \\
0 \\
0 \\
0 \\
0 \\
14\end{array}$ & $\begin{array}{r}1,130 \\
-26 \\
627 \\
379 \\
-\mathrm{N} \\
\frac{\mathrm{N}}{98}\end{array}$ & $\begin{array}{r}913 \\
1 \\
13 \\
492 \\
302 \\
\frac{N}{N} \\
\frac{N}{105}\end{array}$ & $\begin{array}{r}32 \\
\frac{2}{2} \\
\frac{13}{3} \\
\frac{9}{5} \\
-\end{array}$ & $\begin{array}{r}42 \\
0 \\
2 \\
15 \\
7 \\
5 \\
5 \\
1 \\
3 \\
0\end{array}$ & $\begin{array}{r}186 \\
2 \\
9 \\
23 \\
147 \\
19 \\
17 \\
6 \\
17 \\
1\end{array}$ & $\begin{array}{r}1,842 \\
16 \\
112 \\
647 \\
314 \\
252 \\
267 \\
60 \\
169 \\
5\end{array}$ & $\begin{array}{r}1,837 \\
10 \\
98 \\
616 \\
423 \\
261 \\
233 \\
71 \\
122 \\
3\end{array}$ & $\begin{array}{r}29 \\
\frac{-}{-} \\
\frac{1}{1} \\
\frac{8}{17}\end{array}$ & $\begin{array}{r}91 \\
1 \\
0 \\
0 \\
0 \\
0 \\
0 \\
15 \\
33 \\
27\end{array}$ & $\begin{array}{r}860 \\
5 \\
5 \\
0 \\
0 \\
4 \\
0 \\
53 \\
812 \\
70\end{array}$ & $\begin{array}{r}3,773 \\
61 \\
42 \\
- \\
- \\
11 \\
- \\
901 \\
1,441 \\
1,317\end{array}$ & $\begin{array}{r}2,107 \\
28 \\
34 \\
- \\
- \\
- \\
- \\
527 \\
556 \\
962\end{array}$ \\
\hline $\begin{array}{l}\text { E.S. Central } \\
\text { Alabama }^{\dagger} \\
\text { Kentucky } \\
\text { Mississippi } \\
\text { Tennessee }^{\dagger}\end{array}$ & $\begin{array}{l}\bar{N} \\
- \\
-\end{array}$ & $\begin{array}{l}3 \\
0 \\
0 \\
0 \\
3\end{array}$ & $\begin{array}{r}13 \\
0 \\
2 \\
0 \\
13\end{array}$ & $\begin{array}{r}131 \\
\mathrm{~N} \\
- \\
131\end{array}$ & $\begin{array}{r}156 \\
N \\
27 \\
1 \\
128\end{array}$ & $\begin{array}{l}10 \\
2 \\
3 \\
5\end{array}$ & $\begin{array}{r}13 \\
5 \\
1 \\
1 \\
5\end{array}$ & $\begin{array}{r}25 \\
19 \\
8 \\
7 \\
13\end{array}$ & $\begin{array}{r}647 \\
280 \\
63 \\
68 \\
236\end{array}$ & $\begin{array}{r}417 \\
137 \\
46 \\
43 \\
191\end{array}$ & $\frac{-}{\frac{N}{N}}$ & $\begin{array}{l}1 \\
1 \\
0 \\
0 \\
0\end{array}$ & $\begin{array}{r}70 \\
70 \\
0 \\
1 \\
0\end{array}$ & $\begin{array}{r}113 \\
111 \\
\mathrm{~N} \\
2 \\
\mathrm{~N}\end{array}$ & $\begin{array}{r}205 \\
205 \\
\mathrm{~N} \\
\mathrm{~N}\end{array}$ \\
\hline $\begin{array}{l}\text { W.S. Central } \\
\text { Arkansas } \\
\text { Louisiana } \\
\text { Oklahoma } \\
\text { Texas }^{\dagger}\end{array}$ & $\begin{array}{l}- \\
- \\
\mathrm{N} \\
\mathrm{N}\end{array}$ & $\begin{array}{l}0 \\
0 \\
0 \\
0 \\
0\end{array}$ & $\begin{array}{l}5 \\
3 \\
4 \\
0 \\
0\end{array}$ & $\begin{array}{r}20 \\
12 \\
8 \\
\mathrm{~N} \\
\mathrm{~N}\end{array}$ & $\begin{array}{r}104 \\
12 \\
92 \\
\mathrm{~N} \\
\mathrm{~N}\end{array}$ & $\begin{array}{r}21 \\
4 \\
8 \\
1 \\
8\end{array}$ & $\begin{array}{r}29 \\
1 \\
4 \\
1 \\
22\end{array}$ & $\begin{array}{r}52 \\
5 \\
27 \\
6 \\
36\end{array}$ & $\begin{array}{r}1,376 \\
68 \\
255 \\
64 \\
989\end{array}$ & $\begin{array}{r}1,086 \\
45 \\
244 \\
32 \\
765\end{array}$ & $\begin{array}{r}76 \\
5 \\
- \\
71\end{array}$ & $\begin{array}{r}187 \\
9 \\
0 \\
0 \\
170\end{array}$ & $\begin{array}{r}1,757 \\
110 \\
8 \\
0 \\
1,647\end{array}$ & $\begin{array}{r}9,573 \\
739 \\
48 \\
-786\end{array}$ & $\begin{array}{r}5,909 \\
21 \\
119 \\
-769\end{array}$ \\
\hline $\begin{array}{l}\text { Mountain } \\
\text { Arizona } \\
\text { Colorado } \\
\text { Idaho }^{\dagger} \\
\text { Montana }^{\dagger} \\
\text { Nevada }^{\dagger} \\
\text { New Mexico }{ }^{\dagger} \\
\text { Utah } \\
\text { Wyoming }\end{array}$ & $\begin{array}{l}- \\
N \\
N \\
N \\
- \\
- \\
- \\
-\end{array}$ & $\begin{array}{l}1 \\
0 \\
0 \\
0 \\
0 \\
0 \\
0 \\
0 \\
1\end{array}$ & $\begin{array}{l}8 \\
0 \\
0 \\
0 \\
1 \\
0 \\
1 \\
8 \\
4\end{array}$ & $\begin{array}{r}79 \\
N \\
N \\
N \\
- \\
1 \\
36 \\
42\end{array}$ & $\begin{array}{l}47 \\
N \\
N \\
N \\
- \\
- \\
24 \\
23\end{array}$ & $\begin{array}{l}3 \\
2 \\
1 \\
- \\
- \\
- \\
-\end{array}$ & $\begin{array}{l}8 \\
3 \\
1 \\
0 \\
0 \\
1 \\
1 \\
0 \\
0\end{array}$ & $\begin{array}{r}25 \\
16 \\
3 \\
1 \\
1 \\
12 \\
5 \\
2 \\
0\end{array}$ & $\begin{array}{r}360 \\
156 \\
43 \\
2 \\
1 \\
91 \\
58 \\
9 \\
-\end{array}$ & $\begin{array}{r}370 \\
150 \\
42 \\
20 \\
5 \\
97 \\
48 \\
8 \\
-\end{array}$ & $\begin{array}{l}\frac{51}{43} \\
\frac{-}{-} \\
\frac{7}{1}\end{array}$ & $\begin{array}{r}56 \\
0 \\
30 \\
0 \\
0 \\
0 \\
3 \\
13 \\
0\end{array}$ & $\begin{array}{r}138 \\
0 \\
76 \\
0 \\
2 \\
0 \\
34 \\
55 \\
11\end{array}$ & $\begin{array}{r}2,356 \\
1,262 \\
2 \\
- \\
323 \\
716 \\
53\end{array}$ & $\begin{array}{r}2,266 \\
1,580 \\
- \\
- \\
- \\
188 \\
446 \\
52\end{array}$ \\
\hline $\begin{array}{l}\text { Pacific } \\
\text { Alaska } \\
\text { California } \\
\text { Hawaii } \\
\text { Oregon }^{\dagger} \\
\text { Washington }^{-}\end{array}$ & $\begin{array}{l}-\bar{T} \\
\frac{N}{N} \\
N\end{array}$ & $\begin{array}{l}0 \\
0 \\
0 \\
0 \\
0 \\
0\end{array}$ & $\begin{array}{l}0 \\
0 \\
0 \\
0 \\
0 \\
0\end{array}$ & $\begin{array}{l}-\bar{T} \\
\frac{\mathrm{N}}{\mathrm{N}} \\
\mathrm{N}\end{array}$ & $\begin{array}{l}-\bar{T} \\
\frac{\mathrm{N}}{\mathrm{N}} \\
\mathrm{N}\end{array}$ & $\begin{array}{l}\frac{4}{1} \\
-1 \\
1 \\
2\end{array}$ & $\begin{array}{r}34 \\
0 \\
29 \\
0 \\
0 \\
2\end{array}$ & $\begin{array}{r}51 \\
4 \\
41 \\
2 \\
3 \\
10\end{array}$ & $\begin{array}{r}1,423 \\
9 \\
1,225 \\
16 \\
17 \\
156\end{array}$ & $\begin{array}{r}1,563 \\
6 \\
1,390 \\
9 \\
32 \\
126\end{array}$ & $\begin{array}{l}- \\
- \\
N \\
N \\
N\end{array}$ & $\begin{array}{l}0 \\
0 \\
0 \\
0 \\
0 \\
0\end{array}$ & $\begin{array}{l}0 \\
0 \\
0 \\
0 \\
0 \\
0\end{array}$ & $\begin{array}{l}- \\
- \\
N \\
N \\
N\end{array}$ & $\begin{array}{l}- \\
- \\
N \\
N \\
N\end{array}$ \\
\hline $\begin{array}{l}\text { American Samoa } \\
\text { C.N.M.I. } \\
\text { Guam } \\
\text { Puerto Rico } \\
\text { U.S. Virgin Islands }\end{array}$ & $\frac{-}{-}$ & $\begin{array}{l}0 \\
0 \\
0 \\
0 \\
0\end{array}$ & $\begin{array}{l}0 \\
0 \\
0 \\
0 \\
0\end{array}$ & $\frac{-}{-}$ & $\begin{array}{l}- \\
\frac{-}{N} \\
-\end{array}$ & $\begin{array}{l}U \\
\frac{U}{4} \\
-\end{array}$ & $\begin{array}{l}0 \\
0 \\
0 \\
3 \\
0\end{array}$ & $\begin{array}{r}0 \\
0 \\
0 \\
10 \\
0\end{array}$ & $\frac{U}{U} \frac{U}{120}$ & $\begin{array}{r}U \\
U \\
3 \\
193 \\
-\end{array}$ & $\begin{array}{l}U \\
U \\
- \\
-\end{array}$ & $\begin{array}{l}0 \\
0 \\
3 \\
7 \\
0\end{array}$ & $\begin{array}{r}0 \\
0 \\
12 \\
47 \\
0\end{array}$ & $\frac{U}{U} \frac{U}{299}$ & $\begin{array}{r}U \\
U \\
421 \\
616 \\
-\end{array}$ \\
\hline
\end{tabular}

C.N.M.I.: Commonwealth of Northern Mariana Islands.

$\mathrm{U}$ : Unavailable. $\quad-$. No reported cases. $\quad \mathrm{N}$ : Not notifiable.

* Incidence data for reporting year 2006 is provisional.

Cum: Cumulative year-to-date counts. Med: Median. Max: Maximum.

Contains data reported through the National Electronic Disease Surveillance System (NEDSS). 
TABLE II. (Continued) Provisional cases of selected notifiable diseases, United States, weeks ending November 11, 2006, and November 12, 2005 (45th Week)*

\begin{tabular}{|c|c|c|c|c|c|c|c|c|c|c|}
\hline \multirow[b]{4}{*}{ Reporting area } & \multicolumn{10}{|c|}{ West Nile virus disease $^{\dagger}$} \\
\hline & \multicolumn{5}{|c|}{ Neuroinvasive } & \multicolumn{5}{|c|}{ Non-neuroinvasive } \\
\hline & \multirow{2}{*}{$\begin{array}{c}\text { Current } \\
\text { week }\end{array}$} & \multicolumn{2}{|c|}{$\begin{array}{l}\text { Previous } \\
52 \text { weeks }\end{array}$} & \multirow{2}{*}{$\begin{array}{l}\text { Cum } \\
2006\end{array}$} & \multirow{2}{*}{$\begin{array}{l}\text { Cum } \\
2005\end{array}$} & & & $\begin{array}{l}\text { us } \\
\text { ks }\end{array}$ & Cum & Cum \\
\hline & & Med & Max & & & week & Med & Max & 2006 & 2005 \\
\hline United States & - & 1 & 170 & 1,351 & 1,189 & - & 1 & 380 & 2,382 & 1,680 \\
\hline New England & - & 0 & 3 & 9 & 9 & - & 0 & 2 & 3 & 4 \\
\hline Connecticut & - & 0 & 3 & 7 & 4 & - & 0 & 1 & 2 & 2 \\
\hline Maine ${ }^{\S}$ & - & 0 & 0 & - & - & - & 0 & 0 & - & - \\
\hline Massachusetts & - & 0 & 1 & 2 & 4 & - & 0 & 1 & 1 & 2 \\
\hline New Hampshire & - & 0 & 0 & - & - & - & 0 & 0 & - & - \\
\hline Rhode Island & - & 0 & 0 & - & 1 & - & 0 & 0 & - & - \\
\hline Vermont ${ }^{\S}$ & - & 0 & 0 & - & - & - & 0 & 0 & - & - \\
\hline Mid. Atlantic & - & 0 & 6 & 18 & 47 & - & 0 & 3 & 7 & 22 \\
\hline New Jersey & - & 0 & 2 & 2 & 3 & - & 0 & 1 & 2 & 3 \\
\hline New York (Upstate) & - & 0 & 0 & - & 19 & - & 0 & 0 & - & 5 \\
\hline New York City & - & 0 & 4 & 8 & 11 & - & 0 & 2 & 4 & 3 \\
\hline Pennsylvania & - & 0 & 2 & 8 & 14 & - & 0 & 1 & 1 & 11 \\
\hline E.N. Central & - & 0 & 41 & 230 & 258 & - & 0 & 22 & 99 & 156 \\
\hline Illinois & - & 0 & 21 & 117 & 136 & - & 0 & 19 & 70 & 115 \\
\hline Indiana & - & 0 & 7 & 26 & 11 & - & 0 & 2 & 7 & 12 \\
\hline Michigan & - & 0 & 9 & 41 & 54 & - & 0 & 1 & 2 & 8 \\
\hline Ohio & - & 0 & 11 & 35 & 46 & - & 0 & 3 & $1 \overline{1}$ & 15 \\
\hline Wisconsin & - & 0 & 2 & 11 & 11 & - & 0 & 2 & 9 & 6 \\
\hline W.N. Central & - & 0 & 35 & 214 & 169 & - & 0 & 76 & 441 & 463 \\
\hline & - & 0 & 3 & 21 & 14 & - & 0 & 4 & 13 & 23 \\
\hline Kansas & - & 0 & 3 & 17 & 17 & - & 0 & 3 & 13 & $\mathrm{~N}$ \\
\hline Minnesota & - & 0 & 6 & 30 & 18 & - & 0 & 7 & 35 & 27 \\
\hline Missouri & - & 0 & 13 & 47 & 17 & - & 0 & 2 & 12 & 13 \\
\hline Nebraska ${ }^{\S}$ & - & 0 & 8 & 41 & 55 & - & 0 & 35 & 176 & 133 \\
\hline North Dakota & - & 0 & 5 & 20 & 12 & - & 0 & 28 & 117 & 74 \\
\hline South Dakota & - & 0 & 7 & 38 & 36 & - & 0 & 22 & 75 & 193 \\
\hline S. Atlantic & - & 0 & 2 & 13 & 34 & - & 0 & 4 & 7 & 29 \\
\hline Delaware & - & 0 & 0 & - & 1 & - & 0 & 0 & - & 1 \\
\hline District of Columbia & - & 0 & 0 & - & 3 & - & 0 & 1 & 1 & 2 \\
\hline Florida & - & 0 & 1 & 3 & 10 & - & 0 & 0 & - & 11 \\
\hline Georgia & - & 0 & 1 & 2 & 9 & - & 0 & 3 & 5 & 11 \\
\hline Maryland $\$$ & - & 0 & 2 & 7 & 4 & - & 0 & 1 & 1 & 1 \\
\hline North Carolina & - & 0 & 0 & - & 2 & - & 0 & 0 & - & 2 \\
\hline South Carolina ${ }^{\S}$ & - & 0 & 0 & - & 5 & - & 0 & 0 & - & - \\
\hline Virginia ${ }^{\S}$ & - & 0 & 0 & - & - & - & 0 & 0 & - & 1 \\
\hline West Virginia & - & 0 & 1 & 1 & - & $N$ & 0 & 0 & $\mathrm{~N}$ & $N$ \\
\hline E.S. Central & - & 0 & 14 & 106 & 65 & - & 0 & 15 & 92 & 38 \\
\hline Alabama $^{\S}$ & - & 0 & 2 & 7 & 6 & - & 0 & 0 & - & 4 \\
\hline Kentucky & - & 0 & 0 & - & 5 & - & 0 & 1 & 1 & - \\
\hline Mississippi & - & 0 & 10 & 84 & 39 & - & 0 & 15 & 89 & 31 \\
\hline Tennessee ${ }^{\S}$ & - & 0 & 4 & 15 & 15 & - & 0 & 2 & 2 & 3 \\
\hline W.S. Central & - & 0 & 59 & 340 & 156 & - & 0 & 26 & 204 & 149 \\
\hline Arkansas & - & 0 & 4 & 21 & 13 & - & 0 & 2 & 5 & 15 \\
\hline Louisiana & - & 0 & 14 & 88 & - & - & 0 & 9 & 81 & 54 \\
\hline Oklahoma & - & 0 & 6 & 26 & 17 & - & 0 & 4 & 18 & 14 \\
\hline Texas ${ }^{\S}$ & - & 0 & 38 & 205 & 126 & - & 0 & 15 & 100 & 66 \\
\hline Mountain & - & 0 & 61 & 337 & 145 & - & 0 & 222 & 1,300 & 238 \\
\hline Arizona & - & 0 & 9 & 47 & 52 & - & 0 & 12 & 56 & 59 \\
\hline Colorado & - & 0 & 10 & 60 & 21 & - & 0 & 48 & 250 & 85 \\
\hline Idaho§ & - & 0 & 30 & 111 & 3 & - & 0 & 151 & 752 & 10 \\
\hline Montana ${ }^{\S}$ & - & 0 & 3 & 12 & 8 & - & 0 & 7 & 21 & 17 \\
\hline Nevada $\$$ & - & 0 & 9 & 34 & 14 & - & 0 & 13 & 75 & 17 \\
\hline New Mexico§ & - & 0 & 1 & 3 & 20 & - & 0 & 1 & 5 & 13 \\
\hline Utah & - & 0 & 8 & 55 & 21 & - & 0 & 17 & 101 & 31 \\
\hline Wyoming & - & 0 & 7 & 15 & 6 & - & 0 & 8 & 40 & 6 \\
\hline Pacific & - & 0 & 15 & 84 & 306 & - & 0 & 45 & 229 & 581 \\
\hline Alaska & - & 0 & 0 & - & - & - & 0 & 0 & - & - \\
\hline California & - & 0 & 15 & 78 & 305 & - & 0 & 33 & 178 & 575 \\
\hline Hawaii & - & 0 & 0 & - & - & - & 0 & 0 & - & - \\
\hline Oregon ${ }^{\S}$ & - & 0 & 2 & 6 & 1 & - & 0 & 12 & 48 & 6 \\
\hline Washington & - & 0 & 0 & - & - & - & 0 & 2 & 3 & - \\
\hline American Samoa & U & 0 & 0 & U & U & $U$ & 0 & 0 & U & U \\
\hline C.N.M.I. & U & 0 & 0 & $\mathrm{U}$ & U & U & 0 & 0 & U & U \\
\hline Guam & - & 0 & 0 & - & - & - & 0 & 0 & - & - \\
\hline Puerto Rico & - & 0 & 0 & - & - & - & 0 & 0 & - & - \\
\hline U.S. Virgin Islands & - & 0 & 0 & - & - & - & 0 & 0 & - & - \\
\hline
\end{tabular}

C.N.M.I.: Commonwealth of Northern Mariana Islands.

U: Unavailable. - No reported cases. N: Not notifiable. Cum: Cumulative year-to-date counts. Med: Median. Max: Maximum.

* Incidence data for reporting year 2006 is provisional.

Updated weekly from reports to the Division of Vector-Borne Infectious Diseases, National Center for Zoonotic, Vector-Borne, and Enteric Diseases (proposed) (ArboNET Surveillance).

${ }^{\S}$ Contains data reported through the National Electronic Disease Surveillance System (NEDSS). 
TABLE III. Deaths in 122 U.S. cities, ${ }^{*}$ week ending November 11, 2006 (45th Week)

\begin{tabular}{|c|c|c|c|c|c|c|c|c|c|c|c|c|c|c|c|}
\hline \multirow[b]{2}{*}{ Reporting Area } & \multicolumn{6}{|c|}{ All causes, by age (years) } & \multirow[b]{2}{*}{$\begin{array}{l}\text { P\& }^{\dagger} \\
\text { Total }\end{array}$} & \multirow[b]{2}{*}{ Reporting Area } & \multicolumn{6}{|c|}{ All causes, by age (years) } & \multirow[b]{2}{*}{$\begin{array}{l}\text { P\& } I^{\dagger} \\
\text { Tota }\end{array}$} \\
\hline & $\begin{array}{c}\text { All } \\
\text { Ages }\end{array}$ & $\geq 65$ & $45-64$ & $25-44$ & $1-24$ & $<1$ & & & $\begin{array}{c}\text { All } \\
\text { Ages }\end{array}$ & $\geq 65$ & $45-64$ & $25-44$ & $1-24$ & $<1$ & \\
\hline New England & 431 & 294 & 97 & 23 & 6 & 11 & 49 & S. Atlantic & 928 & 559 & 242 & 78 & 32 & 16 & 50 \\
\hline Boston, MA & 125 & 74 & 32 & 7 & 3 & 9 & 12 & Atlanta, GA & 135 & 74 & 39 & 13 & 6 & 3 & 5 \\
\hline Bridgeport, CT & 23 & 18 & 3 & 2 & - & - & 3 & Baltimore, MD & 139 & 76 & 41 & 17 & 2 & 3 & 6 \\
\hline Cambridge, MA & 14 & 13 & 1 & - & - & - & 3 & Charlotte, NC & 105 & 69 & 22 & 9 & 5 & - & 9 \\
\hline Fall River, MA & 24 & 18 & 3 & 3 & - & - & 1 & Jacksonville, FL & 121 & 75 & 30 & 11 & 3 & 2 & 9 \\
\hline Hartford, CT & 35 & 22 & 11 & 1 & 1 & - & 3 & Miami, FL & 38 & 24 & 8 & 4 & 1 & 1 & - \\
\hline Lowell, MA & 24 & 18 & 4 & 1 & 1 & - & 3 & Norfolk, VA & 27 & 17 & 7 & 1 & - & 2 & 2 \\
\hline Lynn, MA & 9 & 6 & 3 & - & - & - & 2 & Richmond, VA & 45 & 25 & 15 & 4 & 1 & - & 5 \\
\hline New Bedford, MA & 21 & 17 & 3 & 1 & - & - & 5 & Savannah, GA & 50 & 36 & 8 & 5 & 1 & - & 2 \\
\hline New Haven, CT & U & U & U & U & $U$ & U & U & St. Petersburg, FL & 14 & 10 & 3 & 1 & - & - & - \\
\hline Providence, RI & 52 & 35 & 13 & 2 & - & 2 & 10 & Tampa, FL & 140 & 88 & 36 & 7 & 8 & - & 9 \\
\hline Somerville, MA & 5 & 2 & 3 & - & - & - & - & Washington, D.C. & 99 & 55 & 29 & 5 & 5 & 5 & 2 \\
\hline Springfield, MA & 29 & 22 & 7 & - & - & - & - & Wilmington, DE & 15 & 10 & 4 & 1 & - & - & 1 \\
\hline Waterbury, CT & 31 & 22 & 6 & 3 & - & - & 4 & E.S. Central & 737 & 466 & 192 & 42 & 18 & 19 & 49 \\
\hline Worcester, MA & 39 & 27 & 8 & 3 & 1 & - & 3 & Birmingham, AL & 96 & 64 & 26 & 4 & - & 2 & 7 \\
\hline Mid. Atlantic & 1,826 & 1,266 & 384 & 109 & 33 & 34 & 105 & Chattanooga, TN & 84 & 48 & 25 & 4 & 5 & 2 & 3 \\
\hline Albany, NY & 56 & 39 & 11 & 3 & 2 & 1 & 5 & Knoxville, TN & 103 & 68 & 27 & 3 & 3 & 2 & 7 \\
\hline Allentown, PA & 26 & 19 & 5 & 1 & 1 & - & 1 & Lexington, $\mathrm{KY}$ & 63 & 39 & 16 & 6 & 1 & 1 & 6 \\
\hline Buffalo, NY & 84 & 51 & 26 & 4 & 2 & 1 & 2 & Memphis, TN & 125 & 79 & 37 & 3 & 2 & 4 & 10 \\
\hline Camden, NJ & 30 & 16 & 9 & 4 & 1 & - & 1 & Mobile, AL & 63 & 41 & 12 & 9 & 1 & - & - \\
\hline Elizabeth, NJ & 13 & 9 & 2 & 2 & - & - & - & Montgomery, AL & 64 & 44 & 13 & 2 & 1 & 4 & 6 \\
\hline Erie, PA & 30 & 24 & 5 & - & - & 1 & 2 & Nashville, TN & 139 & 83 & 36 & 11 & 5 & 4 & 10 \\
\hline Jersey City, NJ & 38 & 23 & 8 & 6 & - & 1 & 1 & W.S. Central & 1,233 & 779 & 300 & 95 & 28 & 31 & 74 \\
\hline New York City, NY & 799 & 557 & 176 & 47 & 11 & 8 & 45 & $\begin{array}{l}\text { W.S. Central } \\
\text { Austin, TX }\end{array}$ & $\begin{array}{r}1,200 \\
67\end{array}$ & 52 & 9 & 5 & - & $\begin{array}{r}01 \\
1\end{array}$ & $\begin{array}{r}74 \\
6\end{array}$ \\
\hline Newark, NJ & 50 & 27 & 16 & 4 & 2 & 1 & 5 & $\begin{array}{l}\text { Austın, IX } \\
\text { Baton Rouge, LA }\end{array}$ & 36 & 22 & 13 & - & - & 1 & 3 \\
\hline Paterson, NJ & 10 & 8 & 1 & - & 1 & - & - & $\begin{array}{l}\text { Baton Rouge, LA } \\
\text { Corpus Christi. TX }\end{array}$ & $\begin{array}{l}50 \\
55\end{array}$ & 40 & 11 & $\overline{2}$ & $\overline{2}$ & - & 7 \\
\hline Philadelphia, PA & 315 & 211 & 53 & 22 & 10 & 19 & 14 & $\begin{array}{l}\text { Corpus Cnristl, IX } \\
\text { Dallas, TX }\end{array}$ & 192 & 104 & 50 & 24 & 6 & 8 & 12 \\
\hline Pittsburgh, $\mathrm{PA}^{\S}$ & 29 & 17 & 8 & 4 & - & - & 2 & $\begin{array}{l}\text { Dallas, IX } \\
\text { EIPaso. TX }\end{array}$ & $\begin{array}{r}192 \\
75\end{array}$ & $\begin{array}{r}104 \\
52\end{array}$ & 20 & $\begin{array}{r}24 \\
2\end{array}$ & $\begin{array}{l}0 \\
1\end{array}$ & $\underline{0}$ & $\begin{array}{r}12 \\
6\end{array}$ \\
\hline Reading, PA & 30 & 24 & 5 & 1 & - & - & - & El Paso, IX & 113 & $\begin{array}{l}52 \\
81\end{array}$ & 20 & $\begin{array}{l}2 \\
6\end{array}$ & - & - & $\begin{array}{l}6 \\
7\end{array}$ \\
\hline Rochester, NY & 106 & 83 & 17 & 3 & 1 & 2 & 10 & $\begin{array}{l}\text { Fort Worth, TX } \\
\text { Houston TX }\end{array}$ & 323 & 191 & $\begin{array}{l}20 \\
86\end{array}$ & $\begin{array}{r}0 \\
27\end{array}$ & $\overline{12}$ & 7 & 11 \\
\hline Schenectady, NY & 18 & 17 & 1 & - & - & - & 2 & Houston, TX & $\begin{array}{r}323 \\
64\end{array}$ & 41 & $\begin{array}{l}86 \\
16\end{array}$ & $\begin{array}{r}27 \\
3\end{array}$ & $\begin{array}{r}12 \\
2\end{array}$ & 2 & $\begin{array}{r}11 \\
1\end{array}$ \\
\hline Scranton, PA & 38 & 26 & 11 & 1 & - & - & 6 & $\begin{array}{l}\text { Little Rock, AR } \\
\text { New Orleans }\end{array}$ & $\begin{array}{r}64 \\
U\end{array}$ & 41 & 10 & 3 & U & 2 & $\begin{array}{l}1 \\
\text { U }\end{array}$ \\
\hline Syracuse, NY & 95 & 74 & 17 & 3 & 1 & - & 6 & New Urleans, LA" & 165 & 107 & 32 & U & U & $\begin{array}{l}8 \\
8\end{array}$ & 11 \\
\hline Trenton, NJ & 20 & 9 & 8 & 2 & 1 & - & 1 & $\begin{array}{l}\text { San Antonio, IX } \\
\text { Shreveport IA }\end{array}$ & $\begin{array}{r}165 \\
45\end{array}$ & $\begin{array}{r}107 \\
32\end{array}$ & $\begin{array}{r}32 \\
8\end{array}$ & $\begin{array}{r}16 \\
3\end{array}$ & 2 & $\begin{array}{l}8 \\
1\end{array}$ & $\begin{array}{r}11 \\
8\end{array}$ \\
\hline Utica, NY & 16 & 13 & 1 & 2 & - & - & - & Shreveport, LA & $\begin{array}{l}45 \\
98\end{array}$ & $\begin{array}{l}32 \\
57\end{array}$ & $\begin{array}{r}8 \\
29\end{array}$ & $\begin{array}{l}3 \\
7\end{array}$ & $\begin{array}{l}1 \\
2\end{array}$ & $\begin{array}{l}1 \\
3\end{array}$ & $\begin{array}{l}8 \\
2\end{array}$ \\
\hline Yonkers, NY & 23 & 19 & 4 & - & - & - & 2 & Tulsa, OK & & & & & 2 & 3 & 2 \\
\hline E.N. Central & 1,911 & 1,242 & 463 & 121 & 43 & 42 & 94 & Mountain & 960 & 623 & 213 & 70 & 26 & 28 & 61 \\
\hline Akron, $\mathrm{OH}$ & 51 & 32 & 14 & 1 & 2 & 2 & - & Albuquerque, NM & 100 & 59 & 24 & 10 & 7 & - & 2 \\
\hline Canton, $\mathrm{OH}$ & 43 & 37 & 5 & 1 & - & - & 4 & Boise, ID & 43 & 36 & 6 & - & 1 & - & 4 \\
\hline Chicago, IL & 271 & 146 & 83 & 26 & 8 & 8 & 14 & Colorado Springs, CO & 80 & 63 & 10 & 4 & 1 & 2 & 7 \\
\hline Cincinnati, $\mathrm{OH}$ & 67 & 41 & 12 & 60 & $\begin{array}{l}0 \\
4\end{array}$ & $\begin{array}{l}0 \\
4\end{array}$ & $\begin{array}{r}14 \\
4\end{array}$ & Denver, CO & 95 & 55 & 18 & 9 & 4 & 9 & 6 \\
\hline Cleveland, $\mathrm{OH}$ & 253 & 193 & 37 & 14 & $\begin{array}{l}4 \\
5\end{array}$ & 4 & $\begin{array}{r}4 \\
11\end{array}$ & Las Vegas, NV & 217 & 137 & 55 & 14 & 5 & 6 & 12 \\
\hline Columbus, $\mathrm{OH}$ & 204 & 120 & 59 & 14 & 6 & 5 & 12 & Ogden, UT & 31 & 25 & 3 & 2 & 1 & - & 2 \\
\hline Dayton, $\mathrm{OH}$ & 120 & 83 & 27 & 7 & 2 & 1 & 10 & Phoenix, AZ & 132 & 66 & 44 & $1 \overline{3}$ & 2 & 7 & 9 \\
\hline Detroit, MI & 138 & 61 & 57 & 14 & 3 & 3 & 8 & Pueblo, CO & 26 & 19 & 6 & 1 & - & - & 4 \\
\hline Evansville, IN & 49 & 41 & 7 & - & 1 & - & 2 & Salt Like City, UT & 125 & 81 & 27 & 14 & 2 & 1 & 11 \\
\hline Fort Wayne, IN & 57 & 33 & 15 & 3 & 3 & $\overline{3}$ & $\begin{array}{l}2 \\
2\end{array}$ & Tucson, AZ & 111 & 82 & 20 & 3 & 3 & 3 & 4 \\
\hline Gary, IN & 15 & 9 & 4 & 1 & 1 & - & - & Pacific & 1,258 & 842 & 279 & 77 & 28 & 32 & 88 \\
\hline Grand Rapids, MI & 59 & 39 & 12 & 3 & 2 & 3 & - & Berkeley, CA & 16 & 11 & 4 & 1 & - & - & 2 \\
\hline Indianapolis, IN & 190 & 120 & 46 & 14 & 4 & 6 & 12 & Fresno, CA & 89 & 52 & 26 & 8 & 2 & 1 & 6 \\
\hline Lansing, MI & 45 & 34 & 10 & 1 & - & - & 4 & Glendale, CA & 1 & 1 & - & - & - & - & - \\
\hline Milwaukee, WI & 79 & 56 & 15 & 7 & - & 1 & 3 & Honolulu, $\mathrm{HI}$ & 54 & 37 & 11 & 1 & 1 & 4 & 3 \\
\hline Peoria, IL & 42 & 24 & 15 & 2 & - & 1 & 1 & Long Beach, CA & 59 & 41 & 11 & 5 & 2 & 一 & 9 \\
\hline Rockford, IL & 51 & 39 & 9 & 2 & 1 & - & 2 & Los Angeles, CA & 84 & 29 & 31 & 12 & 7 & 5 & 6 \\
\hline South Bend, IN & 46 & 40 & 5 & 1 & - & - & 3 & Pasadena, CA & 43 & 31 & 7 & 3 & 2 & - & 5 \\
\hline Toledo, $\mathrm{OH}$ & 100 & 67 & 28 & 3 & 1 & 1 & 1 & Portland, OR & 128 & 85 & 28 & 6 & 3 & 6 & 4 \\
\hline Youngstown, $\mathrm{OH}$ & 31 & 27 & 3 & 1 & - & - & 1 & Sacramento, CA & 169 & 126 & 32 & 8 & 1 & 2 & 10 \\
\hline W.N. Central & 562 & 367 & 127 & 36 & 17 & 15 & 37 & San Diego, CA & 97 & 68 & 14 & 7 & 4 & 4 & 9 \\
\hline Des Moines, IA & 82 & 56 & 16 & 4 & 2 & 4 & 11 & San Francisco, CA & 115 & 84 & 23 & 7 & - & 1 & 9 \\
\hline Duluth, MN & 32 & 23 & 7 & 1 & 1 & - & 2 & San Jose, CA & 161 & 110 & 40 & 4 & 1 & 6 & 8 \\
\hline Kansas City, KS & 18 & 12 & 4 & 1 & 1 & - & 2 & Santa Cruz, CA & 20 & 13 & 6 & 1 & - & - & 3 \\
\hline Kansas City, MO & 91 & 61 & 20 & 6 & 4 & - & $\overline{6}$ & Seattle, WA & 89 & 62 & 17 & 6 & 2 & 2 & 6 \\
\hline Lincoln, NE & 21 & 14 & 5 & 1 & $\begin{array}{l}4 \\
1\end{array}$ & - & $\begin{array}{l}6 \\
2\end{array}$ & Spokane, WA & 52 & 34 & 14 & 3 & - & 1 & 3 \\
\hline Minneapolis, MN & 58 & 33 & 16 & 5 & 4 & - & 5 & Tacoma, WA & 81 & 58 & 15 & 5 & 3 & - & 5 \\
\hline Omaha, NE & 76 & 56 & 14 & 4 & - & 2 & 7 & Total & $9,846^{\star *}$ & 6,438 & 2,297 & 651 & 231 & 228 & 607 \\
\hline St. Louis, MO & 95 & 54 & 28 & 7 & 2 & 4 & 2 & & & & & & & & \\
\hline St. Paul, MN & 41 & 22 & 9 & 4 & 1 & 5 & 1 & & & & & & & & \\
\hline Wichita, KS & 48 & 36 & 8 & 3 & 1 & - & 1 & & & & & & & & \\
\hline
\end{tabular}

\section{U: Unavailable. - $\quad$ No reported cases.}

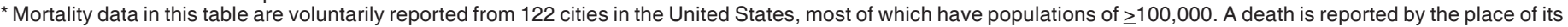
occurrence and by the week that the death certificate was filed. Fetal deaths are not included.

$\uparrow$ Pneumonia and influenza.

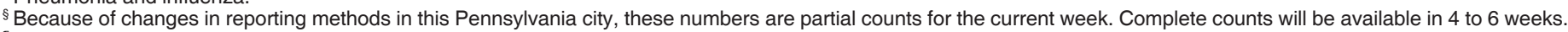

" Because of Hurricane Katrina, weekly reporting of deaths has been temporarily disrupted.

** Total includes unknown ages. 
FIGURE I. Selected notifiable disease reports, United States, comparison of provisional 4-week totals November 11, 2006, with historical data

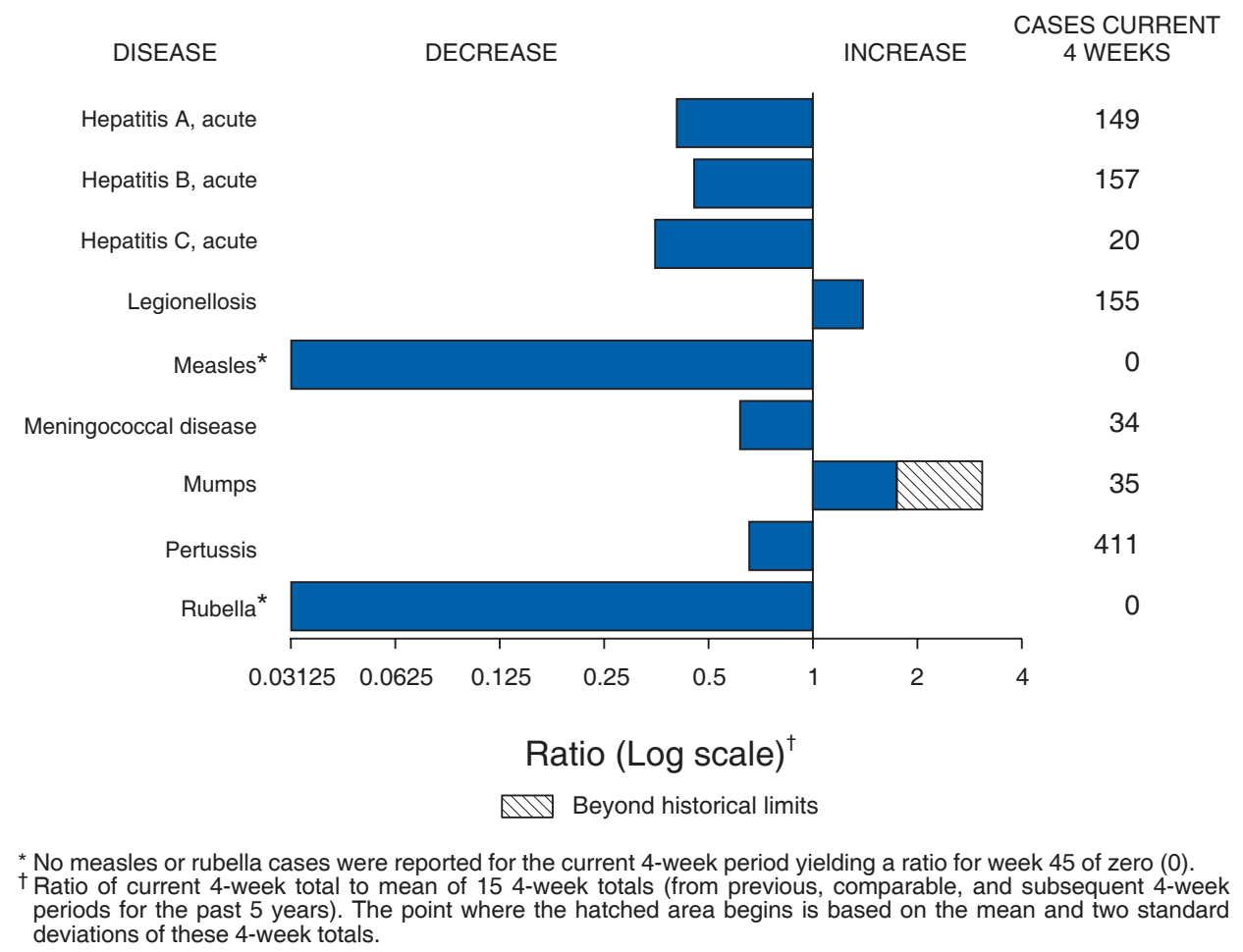

Notifiable Disease Data Team and 122 Cities Mortality Data Patsy A. Hall

Deborah A. Adams Rosaline Dhara

Willie J. Anderson Vernitta Love

Lenee Blanton Pearl C. Sharp 
The Morbidity and Mortality Weekly Report (MMWR) Series is prepared by the Centers for Disease Control and Prevention (CDC) and is available free of charge in electronic format. To receive an electronic copy each week, send an e-mail message to listserv@listserv.cdc.gov. The body content should read SUBscribe mmwrtoc. Electronic copy also is available from CDC's Internet server at http://www.cdc.gov/mmwr or from CDC's file transfer protocol server at $f t p: / / f t p . c d c$.gov/pub/ publications/mmwr. Paper copy subscriptions are available through the Superintendent of Documents, U.S. Government Printing Office, Washington, DC 20402; telephone 202-512-1800.

Data in the weekly MMWR are provisional, based on weekly reports to CDC by state health departments. The reporting week concludes at close of business on Friday; compiled data on a national basis are officially released to the public on the following Friday. Data are compiled in the National Center for Public Health Informatics, Division of Integrated Surveillance Systems and Services. Address all inquiries about the $M M W R$ Series, including material to be considered for publication, to Editor, MMWR Series, Mailstop E-90, CDC, 1600 Clifton Rd., N.E., Atlanta, GA 30333 or to www.mmwrq@cdc.gov.

All material in the MMWR Series is in the public domain and may be used and reprinted without permission; citation as to source, however, is appreciated.

Use of trade names and commercial sources is for identification only and does not imply endorsement by the U.S. Department of Health and Human Services.

References to non-CDC sites on the Internet are provided as a service to $M M W R$ readers and do not constitute or imply endorsement of these organizations or their programs by CDC or the U.S. Department of Health and Human Services. CDC is not responsible for the content of these sites. URL addresses listed in $M M W R$ were current as of the date of publication. 